\title{
GEOTHERMAL MEASUREMENTS IN THE NORTHERN RED SEA: IMPLICATIONS FOR LITHOSPHERIC THERMAL STRUCTURE AND MODE OF EXTENSION DURING CONTINENTAL RIFTING
}

\author{
Fernando Martínez ${ }^{1}$ and James R. Cochran
}

Lamont-Doherty Geological Observatory of Columbia University, Palisades, New York

Abstract. The northern Red Sea is a continental rift in the
process of transition from continental to oceanic rifting. We
present 191 new heat flow measurements from the northern
Red Sea forming three traverses across the water covered por-
tion of the rift. The heat flow across the rift systematically in-
creases from values of about $125 \mathrm{~mW} / \mathrm{m}^{2}$ seaward of the
coasts to average values greater than $250 \mathrm{~mW} / \mathrm{m}^{2}$ in the axial
depression. The heat flow measurements are evaluated for
environmental disturbances. These are found to be generally
small. The largest estimated disturbance results from the relief of the seafloor and of the top of a subbottom evaporite layer. The relief on these surfaces can account for the $20 \%$ point to point scatter typically observed in the heat flow measurements. Limits are placed on systematic disturbances to the heat flow pattern across the rift. The estimated largest systematic disturbance results from sediment blanketing which may cause a reduction in the heat flow on the order of $10 \%$. The heat flow variation across the rift is then utilized to examine the lithospheric thermal structure and the geometry and mechanism of extension. We employ a two-dimensional time-dependent numerical technique to follow the advection and diffusion of heat for a simple shear model and various pure shear models of lithospheric extension. The model of simple shear lithospheric extension along a planar shallow dip detachment produces significantly lower than observed heat flow, results in relatively small amounts of thermal thinning of the lithosphere compared to pure shear extension, and does not result in conditions likely to generate melt. The pure shear models with widening or constant width zones of extension also do not match the observed heat flow nor generate partial melt. The modeling study indicates that the actively extending region must become narrower through time in order to match the heat flow high in the center of the rift, generate some degree of partial melt and produce crustal subsidence within the observed width of the rift. A narrowing of the zone of active extension is also consistent with the pattern of tectonic activity inferred from sedimentary structures observed on seismic reflection profiles which indicate an abatement of activity in the marginal areas and intense present-day activity in the axial depression. In all of the models, an additional component of lithospheric heating other than that produced by extension appears necessary to match heat flow values in the marginal and near coastal areas, as well as explain the observed magnitude of rift flank uplift. Several scenarios in which the zone of extension eventually becomes narrow are consistent with the heat flow data alone, including those resulting in seafloor spreading for the past several million years. However, additional information provided by gravity, magnetic, and seismic reflection and refraction data appear to be most consistent with a history in which a period of relatively uniform extension across the rift is followed by a concentration of extension toward the center

\footnotetext{
${ }^{1}$ Now at Hawaii Institute of Geophysics, Honolulu.

\section{Copyright 1989 by the American Geophysical Union.}

Paper number 89JB00636

0148-0227/89/89JB-00636\$05.00
}

of the rift. Such a model retains a small thickness of continental crust in the center of the rift and does not necessitate active seafloor spreading in the northern Red Sea. The three heat flow traverses indicate that maximum lithospheric thinning is occurring within the axial depression in the northern Red Sea which is very close to initiating seafloor spreading. There is evidence that upper crustal extension is locally concentrated in specific areas along the strike of the axial depression forming "deeps" associated with large recent intrusions. These deeps may constitute a precursory stage to seafloor spreading and appear to be nucleating at discrete and regularly spaced sites along the axis of the rift. The deeps are located midway between regularly spaced cross-trending fault zones or accommodation zones which, in the marginal areas, separate sets of fault blocks along strike. Crustal extension appears to be taken up more diffusely in the accommodation zones than in the intervening fault block domains which are characterized by fewer, larger faults. As a result, initial crustal rupturing and emplacement of large intrusive bodies occurs halfway between the accommodation zones where more focused crustal extension forms the deeps. Comparison with other rifts suggests that the pattern of fault block domains separated by crosstrending fault zones is a common feature, that it originates early in the development of a rift, and that it may continue to influence the development of the rift up to the establishment of a continuous seafloor spreading axis.

\section{Introduction}

The development of a continental rift is in large part controlled by its evolving thermal structure. It is known from experimental and theoretical studies [Murrell, 1976; Goetze and Evans, 1979; Kirby, 1983] that the mechanical strength of the lithosphere, and thus the manner in which the extension is accommodated, is strongly dependent on the temperature structure. Therefore even if the extension producing the rifting results from plate forces applied at some distance from the rift, to understand the rifting process, it is necessary to understand the thermal evolution of the rift. Since the thermal anomaly associated with rifting is a transient feature and rapidly decays following the cessation of rifting or the beginning of seafloor spreading, studies of the structure [e.g., Montadert et al., 1979] or subsidence history [e.g. Watts and Steckler, 1981] of older rifts or continental margins have not been able to examine the actual rifting process in much detail.

The northern Red Sea is a nearly ideal location for a thermal study of active continental rifting and the early stages of continental margin formation for several reasons. First, continental rifting in the northern Red Sea has progressed nearly to the point of initiating seafloor spreading. Thus sufficient extension has occurred (approximately $135 \mathrm{~km}$ ) [Cochran, 1983a; $\mathrm{LePi}$ chon and Francheteau, 1978; Joffe and Garfunkel, 1987; Steckler et al., 1988] to generate a clear and distinct thermal anomaly well above background levels and above the variations caused by environmental, topographic, and sedimentary disturbances to the surface heat flow. Second, the region is located within what was the stable interior of the Afro-Arabian platform and appears to have had a relatively simple thermal history since its consolidation in Pan-African time (circa 500 $\mathrm{Ma}$ ) until the initiation of the current rifting event in the late 
Oligocene or early. Miocene. Third, it is sufficiently far from the thermal anomaly of the Afar hot spot to be unaffected by it. Fourth, a progression exists in the development of the rift from south to north. Greater extension to the south has resulted in the development of a continuous seafloor spreading axis in the southern Red Sea, which becomes discontinuous to the north and finally disappears totally north of $25^{\circ} \mathrm{N}$ where recent studies [Martínez and Cochran, 1988; Guennoc et al., 1988] indicate that continental rifting is still occurring. This progression allows an observation of the rifting process from the more advanced to less advanced stages. Finally, the overall geologic and kinematic history of the Red Sea opening is relatively well established [Freund et al., 1970; LePichon and Francheteau, 1978; Garfunkel, 1981; Cochran, 1983a; Joffe and Garfunkel, 1987; Courtillot et al., 1987] and not overly complicated, providing good constraints for modeling studies. Thus the northern Red Sea can be viewed as a relatively pure example of the development of continental rifting nearly to the point of initiation of seafloor spreading without a complicated tectonic or kinematic history and without the overprint of large thermal signatures from other causes.

In this paper, we present 191 new heat flow measurements from the northern Red Sea. These data form three traverses across the water covered portion of the rift. The environmental, topographic, sedimentary, and other disturbances to the heat flow measurements are assessed, and bounds are placed on the lithospheric thermal anomaly. The lithospheric thermal anomaly is then used as an important constraint for numerical models of lithospheric extension. We examine the viability of various geometries and histories of rifting as mechanisms for lithospheric extension in the northern Red Sea in terms of their ability to account for the observed heat flow pattern and to produce crustal thinning and subsidence/uplift similar to that observed within the rift and whether they are capable of generating partial melt (i.e., to go over to seafloor spreading, as is now occurring). Finally, we combine the information on the form of lithospheric extension derived from the numerical calculations with other geophysical data from the northern Red Sea to discuss geologic models for rifting and the transition to seafloor spreading.

\section{Geological Setting of the Red Sea Rift}

The Red Sea forms part of a late Cenozoic rift system that also includes the Gulf of Aden and East African rifts to the south and the Gulf of Suez and Gulf of Aqaba to the north. Prior to the initiation of rifting in late Oligocene or early Miocene time, this region was contained within the stable platform of the Afro-Arabian land mass. This continental platform formed in Pan-African time through the emplacement of exotic terrains separated by ophiolites [Engel et al., 1980; Stern, 1981; Stoeser and Camp, 1985] and by episodes of granitic plutonism [Stern et al., 1984], resulting in a heterogeneous basement composition.

Sedimentation was nearly continuous throughout the Phanerozoic along the Tethyan marginal shelves to the north of the Afro-Arabian shield with continental "Nubian Sandstone" sediments deposited in the interior. The shield experienced some gentle uplift and erosion during the late Paleozoic and Mesozoic, resulting in the exposure of basement rocks in the areas that are now central Saudi Arabia and eastern Sudan [Bohannon et al., 1989]. Epiric marine sediments and soil horizons of Late Cretaceous to early Oligocene age indicate that Afro-Arabia remained near sea level during that time interval [Bohannon et al., 1989].

Middle Tertiary to Recent alkaline volcanism extends along a north-south trend from East Africa to Jordan [Coleman et al., 1983]. The earliest volcanism predates the start of rifting but is not associated with doming in the Red Sea region [Coleman, 1984; Bohannon et al., 1989] nor does the volcanism follow the north-northwest Red Sea trend. It is, for the most part, located well east of the rift on the Arabian platform [Coleman et al., 1983]. It thus appears that the northern Red Sea region was near sea level at the time of rifting and did not experience an initial doming episode prior to rifting.

The Red Sea rift appears to have formed at the end of the Oligocene or during the early Miocene. Rift shoulder uplift and the deposition of coarse clastics within the subsiding basin appears to have begun shortly after the onset of rifting [Garfunkel and Bartov, 1977; Steckler, 1985]. By the middle Miocene, the rift was cut off from the Mediterranean except for intermittent marine invasions which appear to have extended continuously from the Gulf of Suez to nearly the southern end of the Red Sea, resulting in the deposition of a thick evaporite sequence [Heybroek, 1965]. Open marine conditions were established in latest Miocene or early Pliocene by the opening of a connection to the Indian Ocean through the Straits of Babal-Mandab at the southern tip of the Red Sea. This ended the period of evaporite deposition and signaled the start of marine deposition, which continues to the present.

The present Red Sea rift can be divided into several sections each representing a distinct tectonic stage in the evolution of the rift [Cochran, 1983a]. The southern Red Sea from about $15^{\circ} \mathrm{N}$ to $20^{\circ} \mathrm{N}$ is undergoing seafloor spreading within a well defined "axial trough" [Bäcker et al., 1975] having lineated magnetic anomalies which have been correlated to 4 or $5 \mathrm{Ma}$ near $17^{\circ} \mathrm{N}$ and to 2 or $3 \mathrm{Ma}$ at $20^{\circ} \mathrm{N}$ and at $15^{\circ} \mathrm{N}$ [Roeser, 1975]. The axial trough in this region is about $2000 \mathrm{~m}$ deep, is up to $60 \mathrm{~km}$ wide, and has a rough floor consisting of midocean ridge basalts. North of $20^{\circ} \mathrm{N}$ to about $23.5^{\circ} \mathrm{N}$ the axial trough is discontinuous and forms a series of short isolated trough segments or deeps floored by oceanic basalts [Pautot, 1983; Miller et al., 1985] and associated with lineated magnetic anomalies which have been correlated to less than $2 \mathrm{Ma}$ [Bonatti et al., 1984]. The isolated trough segments occur at regular spacings of about $60-70 \mathrm{~km}$ and are separated by intervening "intertrough zones" that have continuous sedimentary sequences across them [Tramontini and Davies, 1969; Phillips and Ross, 1970; Searle and Ross, 1975] and are associated with low-amplitude magnetic anomalies that trend roughly perpendicular to the direction of the axis [Searle and Ross, 1975; Izzeldin, 1982]. These isolated trough segments or "deeps" appear to represent cells of organized seafloor spreading separated by regions in which organized seafloor spreading has not yet been established [Cochran, 1983a; Bonatti, 1985].

An axial trough floored by basalts is not present north of about $23.5^{\circ} \mathrm{N}$. Instead, a shallower sediment covered "axial depression" is located near the center of the rift at depths of $1100 \mathrm{~m}$ to $1300 \mathrm{~m}$ [Cochran et al., 1986; Pautot et al., 1986; Martínez and Cochran, 1988; Guennoc et al., 1988]. Possible evidence of localized seafloor spreading within this region has only been reported from Mabahiss Deep at about $25.4^{\circ} \mathrm{N}$ where volcanic outcrops and lineated magnetic anomalies have been observed [Pautot et al., 1986]. However, Guennoc et al. [1988] interpret a detailed survey of Mabahiss Deep in terms of a complicated history involving three distinct tectonic phases with intrusive and extrusive magmatic events occurring over a 3 to $4 \mathrm{~m}$.y. period. It is thus not clear whether this deep results from organized seafloor spreading. North of $25.4^{\circ} \mathrm{N}$, no lineated magnetic anomalies have been observed, and that region appears to have accommodated extension through block faulting in the marginal areas [Cochran, 1983a; Martinez and Cochran, 1988] and recently by more concentrated extension and emplacement of localized intrusions within the axial depression [Cochran et al., 1986; Martinez and Cochran, 1988; Guennoc et al., 1988].

The northern Red Sea has rough seafloor bathymetry which forms distinct levels that are about $20-30 \mathrm{~km}$ wide and which deepen toward the center of the sea across 200 to $300-\mathrm{m}$ steps. The region of stepped or terraced bathymetry forms the "main trough" or "marginal areas" and generally ranges in depth from about 400 to $1100 \mathrm{~m}$ seaward of exceptionally narrow coastal shelves. Gravity data from the region north of $26^{\circ} \mathrm{N}$ have been 
interpreted as delineating a block faulted basement structure which underlies the terraced bathymetry [Martinez and Cochran, 1988] and forms an extension of the block faulted structure observed on land. The gravity pattern forms a series of elongate highs and lows in plan view, which are systematically terminated at roughly $60-\mathrm{km}$ intervals along strike by what have been interpreted as cross-trending fault zones [Martinez and Cochran, 1988]. These fault zones, which are also expressed in the bathymetry, are interpreted by Martinez and Cochran [1988] as accommodation zones separating coherent sets of fault blocks.

A deeper "axial depression" at depths of 1100-1300 m exists within the main trough forming a 10 - to $25-\mathrm{km}$-wide zone which extends from Mabahiss Deep to the entrance to the Gulf of Suez. The axial depression has an overall trend of $320^{\circ}$ which is more northwesterly than the overall $335^{\circ}$ trend of the main northern part of the rift. Both the main trough and the axial depression are covered by a sedimentary sequence which includes the middle to late Miocene evaporites and the postMiocene pelagic sediments. Small deeps occur within the axial depression at regular intervals of about $60 \mathrm{~km}$. Some of the deeps have volcanic structures which outcrop at the seafloor, while others are completely sediment covered [Pautot, 1983; Pautot et al., 1984; Cochran et al., 1986; Guennoc et al., 1988]. A pronounced free-air gravity low is found over the length of the axial depression and a triangular shaped -70 $\mathrm{mGal}$ low is located where the axial depression terminates at the triple juncture with the Gulfs of Suez and Aqaba [Martinez and Cochran, 1988]. The magnetic field of the northern Red Sea is made up of low-amplitude, long-wavelength anomalies on which a series of large-amplitude, short-wavelength dipolar anomalies are superimposed. Although a few of the dipolar anomalies occur in the marginal areas, apparently at the intersections of accommodation zones with escarpments marking the edges of the bathymetric terraces, most occur in close proximity to the regularly spaced axial deeps [Martínez and Cochran, 1988; Guennoc et al., 1988].

Seismic reflection profiles in the marginal areas show a general pattern of decreasing sediment disturbance from the deeper to the shallower sediments, while in the axial depression sedimentary disturbances appear to be more intense and to continue to the present [Knott et al., 1966; Phillips and Ross, 1970; Martínez and Cochran, 1988]. Although local sedimentary disturbances and faulting indicate that the marginal areas are not totally quiescent, the pattern of sedimentary deformation suggests that the tectonic activity of the marginal areas is generally decreasing and that tectonic activity is becoming concentrated within the axial depression [Martinez and Cochran, 1988].

\section{Data Acquisition}

In the summer of $1984, \mathrm{R} / \mathrm{V}$ Robert D. Conrad made an extensive geophysical survey of the northern Red Sea between latitudes $26^{\circ} \mathrm{N}$ and $28^{\circ} \mathrm{N}$ [Martinez and Cochran, 1988] (Figure 1). As part of this survey, 191 temperature gradient measurements were obtained along with 10 piston core samples used for thermal conductivity determinations. The heat flow stations were organized into three transects oriented at $060^{\circ}$, approximately perpendicular to the trend of the rift and separated by about $70 \mathrm{~km}$ from each other along the rift. Individual stations within each transect were located about $2 \mathrm{~km}$ apart and each transect consisted of 60-70 stations. This geometry provides three independent measures of the heat flow variation across the rift and furnishes a sample of along-strike variations. The location of the gradient measurements and of the piston core conductivity sites is shown in Figure 2, and their positions and values are given in Tables 1 and 2 .

The temperature gradient measurements were made using a 5-m-long probe equipped with eight thermistors, seven of which are arranged along the exterior length of the probe in an outrigger fashion and the eighth is located on top of the appa-

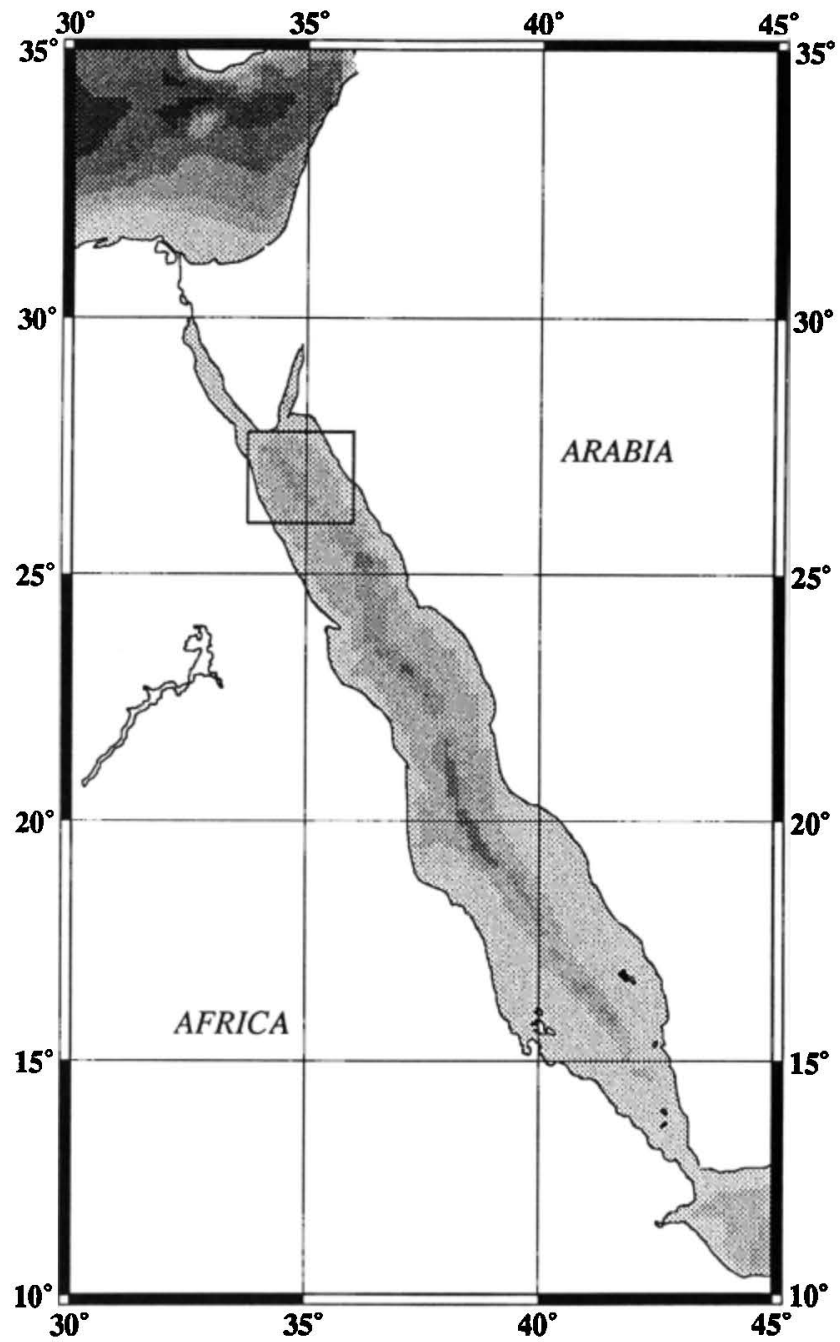

Fig. 1. Map of Red Sea region. Areas below sea level are shaded with the intensity of shading increasing at $500-\mathrm{m}$ intervals. Data are $5^{\prime} \times 5^{\prime}$ gridded topographic measurements (DBDB5) provided by Defense Mapping Agency. Box outlines area of Figure 2 and shows location of the field area.

ratus above the core head in order to measure the bottom water temperature. The instrument was lowered to within a few tens of meters above the seafloor, allowed to equilibrate with the bottom water and then dropped into the bottom sediments. On penetration, the thermistors become frictionally heated and are therefore left in place for several minutes in order to record their cooling behavior from which the equilibrium temperature in the sediments is determined. The instrument package on top of the probe acoustically telemeters information back to the ship via a $12-\mathrm{kHz}$ transponder, so that the tilt of the instrument and the cooling behavior of the thermistors are known in real time and it also records this information internally on magnetic tape. The absolute accuracy of the thermistors is $0.1^{\circ} \mathrm{C}$, but their relative precision is $0.0015^{\circ} \mathrm{C}$. The temperature gradient was therefore determined from the relative change in temperature between the temperature that each probe recorded in the water immediately above the bottom and its equilibrium temperature in the sediments.

Thermal conductivities were determined from a total of ten 6$\mathrm{m}$-long piston cores which were located along each of the heat flow transects in order to sample the spatial distribution of conductivity from the shallower marginal areas to the deeper axial depression (see Figure 2). Thermal conductivities were 


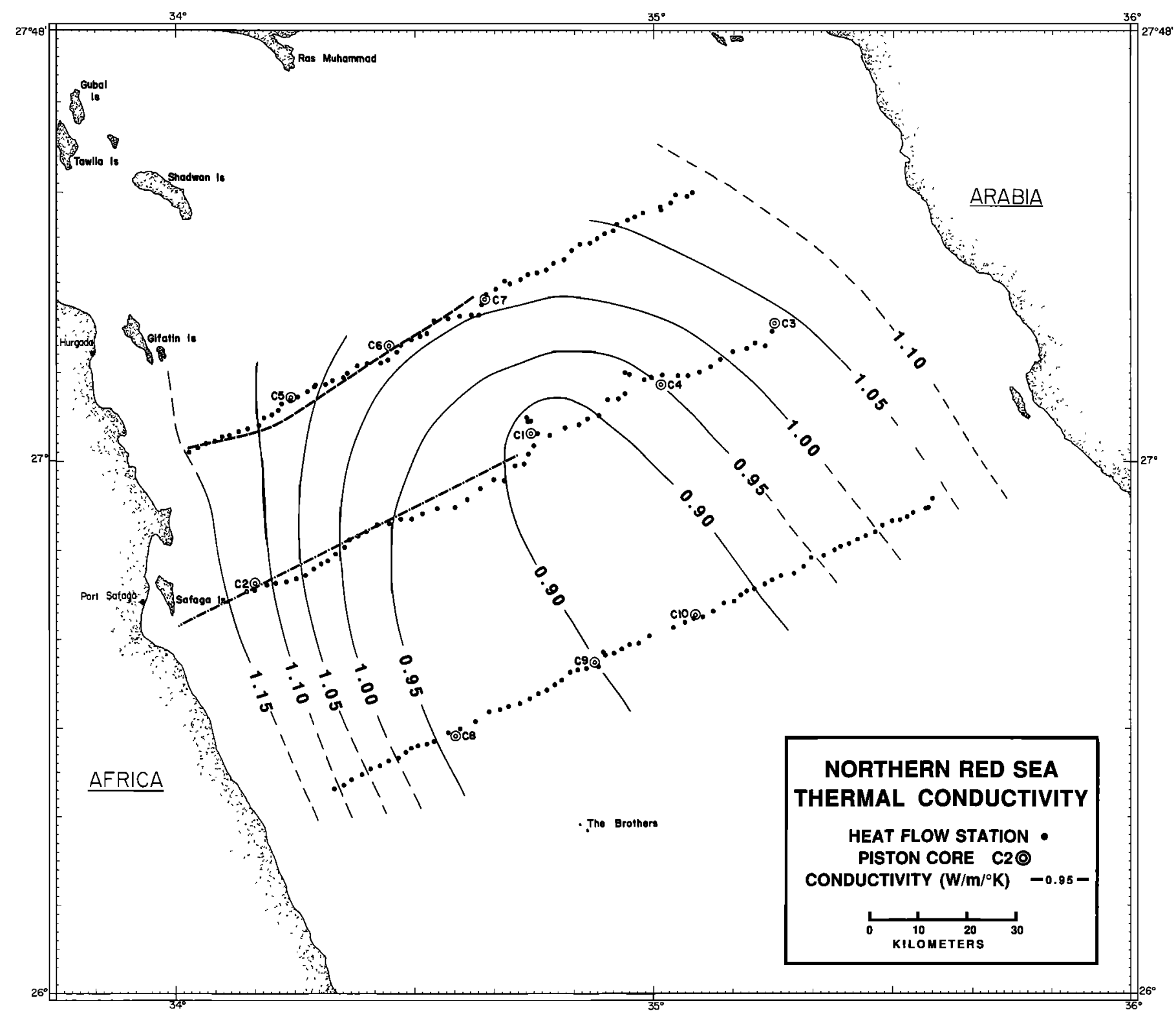

Fig. 2. Location map of heat flow stations and of piston cores used for thermal conductivity measurements. Thermal conductivity values are shown contoured at $0.05 \mathrm{~W} \mathrm{~m}^{-1}{ }^{\circ} \mathrm{K}^{-1}$ intervals. Dashed line along western half of northern heat flow traverse indicates location of seismic line shown in Figure 12. Dash-dotted line along western half of central heat flow traverse indicated location of multichannel seismic line ERS74. 
TABLE 1a. Summary of Heat Flow Measurements: Northern Line

\begin{tabular}{|c|c|c|c|c|c|c|}
\hline Station & $\begin{array}{l}\text { Latitude } \\
\qquad \mathrm{N}\end{array}$ & $\underset{\mathrm{E}}{\text { Longitude }}$ & $\begin{array}{l}\text { Heat Flow, } \\
\mathrm{mW} \mathrm{m}^{-2}\end{array}$ & $\begin{array}{l}\text { Conductivity, } \\
\mathrm{W} \mathrm{m}^{-1}{ }^{\circ} \mathrm{K}^{-1}\end{array}$ & $\begin{array}{l}\text { Gradient, } \\
\mathrm{m}^{\circ} \mathrm{K} \mathrm{m}^{-1}\end{array}$ & $\begin{array}{l}\text { Water Depth, } \\
\text { m }\end{array}$ \\
\hline $\begin{array}{l}5 \mathrm{a} \\
5 \mathrm{~b} \\
5 \mathrm{c} \\
5 \mathrm{~d} \\
5 \mathrm{e} \\
5 \mathrm{f} \\
6 \mathrm{a} \\
6 \mathrm{~b} \\
6 \mathrm{c} \\
6 \mathrm{~d} \\
6 \mathrm{e} \\
6 \mathrm{f} \\
6 \mathrm{~g} \\
6 \mathrm{~h} \\
6 \mathrm{i} \\
6 \mathrm{j} \\
6 \mathrm{k} \\
6 \mathrm{~d} \\
6 \mathrm{~m} \\
6 \mathrm{n} \\
6 \mathrm{o} \\
6 \mathrm{p} \\
6 \mathrm{q} \\
7 \mathrm{a} \\
7 \mathrm{~b} \\
7 \mathrm{c} \\
7 \mathrm{~d} \\
7 \mathrm{e} \\
7 \mathrm{f} \\
7 \mathrm{~g} \\
7 \mathrm{~h} \\
8 \mathrm{a} \\
8 \mathrm{~b} \\
8 \mathrm{c} \\
8 \mathrm{~d} \\
8 \mathrm{e} \\
8 \mathrm{f} \\
8 \mathrm{~g} \\
8 \mathrm{~h} \\
9 \mathrm{a} \\
9 \mathrm{~b} \\
9 \mathrm{c} \\
9 \mathrm{~d} \\
9 \mathrm{e} \\
9 \mathrm{f} \\
9 \mathrm{~g} \\
9 \mathrm{~h} \\
9 \mathrm{i} \\
9 \mathrm{j} \\
9 \mathrm{k} \\
9 \mathrm{l} \\
9 \mathrm{~m}\end{array}$ & $\begin{array}{l}27^{\circ} 29.93^{\prime} \\
27^{\circ} 29.52^{\prime} \\
27^{\circ} 29.56^{\prime} \\
27^{\circ} 28.75^{\prime} \\
27^{\circ} 28.28^{\prime} \\
27^{\circ} 27.96^{\prime} \\
27^{\circ} 27.64^{\prime} \\
27^{\circ} 27.20^{\prime} \\
27^{\circ} 26.83^{\prime} \\
27^{\circ} 26.34^{\prime} \\
27^{\circ} 25.57^{\prime} \\
27^{\circ} 25.28^{\prime} \\
27^{\circ} 24.71^{\prime} \\
27^{\circ} 24.31^{\prime} \\
27^{\circ} 24.16^{\prime} \\
27^{\circ} 23.46^{\prime} \\
27^{\circ} 22.37^{\prime} \\
27^{\circ} 21.94^{\prime} \\
27^{\circ} 21.26^{\prime} \\
27^{\circ} 20.92^{\prime} \\
27^{\circ} 20.77^{\prime} \\
27^{\circ} 20.15^{\prime} \\
27^{\circ} 19.73^{\prime} \\
27^{\circ} 20.00^{\prime} \\
27^{\circ} 19.10^{\prime} \\
27^{\circ} 18.52^{\prime} \\
27^{\circ} 17.44^{\prime} \\
27^{\circ} 16.25^{\prime} \\
27^{\circ} 16.35^{\prime} \\
27^{\circ} 16.12^{\prime} \\
27^{\circ} 15.87^{\prime} \\
27^{\circ} 15.69^{\prime} \\
27^{\circ} 14.19^{\prime} \\
27^{\circ} 13.94^{\prime} \\
27^{\circ} 13.49^{\prime} \\
27^{\circ} 12.95^{\prime} \\
27^{\circ} 12.23^{\prime} \\
27^{\circ} 11.27^{\prime} \\
27^{\circ} 11.28^{\prime} \\
27^{\circ} 10.96^{\prime} \\
27^{\circ} 10.85^{\prime} \\
27^{\circ} 10.69^{\prime} \\
27^{\circ} 9.74^{\prime} \\
27^{\circ} 9.34^{\prime} \\
27^{\circ} 8.96^{\prime} \\
27^{\circ} 8.55^{\prime} \\
27^{\circ} 8.35^{\prime} \\
27^{\circ} 8.31^{\prime} \\
27^{\circ} 7.69^{\prime} \\
27^{\circ} 7.11^{\prime} \\
27^{\circ} 6.67^{\prime} \\
27^{\circ} 6.38^{\prime}\end{array}$ & $\begin{array}{l}35^{\circ} 4.84^{\prime} \\
35^{\circ} 4.15^{\prime} \\
35^{\circ} 2.63^{\prime} \\
35^{\circ} 2.09^{\prime} \\
35^{\circ} 0.89^{\prime} \\
35^{\circ} 0.96^{\prime} \\
34^{\circ} 58.69^{\prime} \\
34^{\circ} 57.66^{\prime} \\
34^{\circ} 56.75^{\prime} \\
34^{\circ} 55.43^{\prime} \\
34^{\circ} 54.93^{\prime} \\
34^{\circ} 53.95^{\prime} \\
34^{\circ} 52.94^{\prime} \\
34^{\circ} 51.95^{\prime} \\
34^{\circ} 50.76^{\prime} \\
34^{\circ} 49.77^{\prime} \\
34^{\circ} 48.77^{\prime} \\
34^{\circ} 47.39^{\prime} \\
34^{\circ} 46.51^{\prime} \\
34^{\circ} 45.31^{\prime} \\
34^{\circ} 44.15^{\prime} \\
34^{\circ} 43.24^{\prime} \\
34^{\circ} 41.92^{\prime} \\
34^{\circ} 41.21^{\prime} \\
34^{\circ} 40.14^{\prime} \\
34^{\circ} 38.95^{\prime} \\
34^{\circ} 38.36^{\prime} \\
34^{\circ} 38.04^{\prime} \\
34^{\circ} 37.05^{\prime} \\
34^{\circ} 35.74^{\prime} \\
34^{\circ} 34.12^{\prime} \\
34^{\circ} 32.33^{\prime} \\
34^{\circ} 14.19^{\prime} \\
34^{\circ} 30.65^{\prime} \\
34^{\circ} 29.44^{\prime} \\
34^{\circ} 28.95^{\prime} \\
34^{\circ} 27.71^{\prime} \\
34^{\circ} 26.93^{\prime} \\
34^{\circ} 26.51^{\prime} \\
34^{\circ} 25.67^{\prime} \\
34^{\circ} 23.94^{\prime} \\
34^{\circ} 22.72^{\prime} \\
34^{\circ} 21.45^{\prime} \\
34^{\circ} 21.07^{\prime} \\
34^{\circ} 19.62^{\prime} \\
34^{\circ} 18.82^{\prime} \\
34^{\circ} 17.52^{\prime} \\
34^{\circ} 17.41^{\prime} \\
34^{\circ} 16.49^{\prime} \\
34^{\circ} 15.24^{\prime} \\
34^{\circ} 14.36^{\prime} \\
34^{\circ} 13.31^{\prime}\end{array}$ & $\begin{array}{l}137 \pm 16.5 \\
129 \pm 17.2 \\
137 \pm 17.0 \\
149 \pm 18.2 \\
197 \pm 24.3 \\
179 \pm 22.3 \\
128 \pm 16.7 \\
171 \pm 23.2 \\
180 \pm 23.5 \\
165 \pm 20.6 \\
177 \pm 25.3 \\
183 \pm 24.1 \\
194 \pm 25.4 \\
216 \pm 28.5 \\
305 \pm 41.9 \\
201 \pm 28.8 \\
174 \pm 22.5 \\
235 \pm 33.7 \\
248 \pm 38.9 \\
270 \pm 39.1 \\
296 \pm 42.8 \\
262 \pm 34.8 \\
184 \pm 23.9 \\
167 \pm 22.6 \\
209 \pm 26.9 \\
164 \pm 25.9 \\
173 \pm 21.9 \\
209 \pm 32.7 \\
179 \pm 23.5 \\
311 \pm 40.4 \\
297 \pm 43.4 \\
296 \pm 39.2 \\
335 \pm 45.2 \\
368 \pm 50.8 \\
369 \pm 50.4 \\
335 \pm 42.1 \\
175 \pm 24.5 \\
428 \pm 59.8 \\
347 \pm 45.8 \\
270 \pm 34.9 \\
232 \pm 31.2 \\
249 \pm 32.2 \\
187 \pm 23.9 \\
196 \pm 25.7 \\
195 \pm 29.3 \\
225 \pm 28.6 \\
163 \pm 28.7 \\
182 \pm 25.6 \\
166 \pm 21.5 \\
135 \pm 17.4 \\
134 \pm 16.3 \\
111 \pm 14.4\end{array}$ & $\begin{array}{l}1.08 \\
1.08 \\
1.08 \\
1.07 \\
1.07 \\
1.07 \\
1.06 \\
1.06 \\
1.05 \\
1.05 \\
1.05 \\
1.05 \\
1.04 \\
1.04 \\
1.04 \\
1.04 \\
1.03 \\
1.03 \\
1.03 \\
1.03 \\
1.02 \\
1.02 \\
1.02 \\
1.02 \\
1.02 \\
1.01 \\
1.01 \\
1.01 \\
1.01 \\
1.01 \\
1.01 \\
1.02 \\
1.02 \\
1.02 \\
1.02 \\
1.02 \\
1.02 \\
1.02 \\
1.02 \\
1.02 \\
1.03 \\
1.03 \\
1.04 \\
1.04 \\
1.05 \\
1.05 \\
1.06 \\
1.06 \\
1.06 \\
1.07 \\
1.08 \\
1.08\end{array}$ & $\begin{array}{l}127 \pm 1.2 \\
119 \pm 2.7 \\
127 \pm 1.6 \\
139 \pm 1.4 \\
184 \pm 2.1 \\
167 \pm 2.1 \\
121 \pm 2.1 \\
161 \pm 3.7 \\
171 \pm 2.8 \\
157 \pm 1.7 \\
169 \pm 4.8 \\
174 \pm 3.6 \\
187 \pm 2.8 \\
208 \pm 3.4 \\
293 \pm 6.5 \\
193 \pm 5.4 \\
169 \pm 2.2 \\
228 \pm 6.2 \\
241 \pm 9.7 \\
262 \pm 7.4 \\
290 \pm 7.8 \\
257 \pm 3.9 \\
180 \pm 2.3 \\
164 \pm 2.9 \\
205 \pm 2.3 \\
162 \pm 6.4 \\
171 \pm 1.4 \\
207 \pm 7.8 \\
177 \pm 2.2 \\
308 \pm 3.4 \\
294 \pm 8.0 \\
290 \pm 4.3 \\
328 \pm 5.7 \\
361 \pm 7.3 \\
362 \pm 6.8 \\
328 \pm 2.7 \\
172 \pm 3.8 \\
420 \pm 9.2 \\
340 \pm 4.9 \\
265 \pm 3.0 \\
225 \pm 4.1 \\
242 \pm 3.1 \\
180 \pm 2.2 \\
188 \pm 3.0 \\
186 \pm 6.6 \\
214 \pm 2.8 \\
154 \pm 9.6 \\
172 \pm 4.7 \\
157 \pm 2.5 \\
127 \pm 2.0 \\
125 \pm 1.2 \\
103 \pm 1.9\end{array}$ & $\begin{array}{r}690 \\
693 \\
717 \\
725 \\
765 \\
765 \\
806 \\
830 \\
835 \\
815 \\
807 \\
820 \\
847 \\
831 \\
857 \\
835 \\
803 \\
833 \\
903 \\
980 \\
985 \\
995 \\
1020 \\
1023 \\
977 \\
1030 \\
1135 \\
1205 \\
1200 \\
1135 \\
1107 \\
1069 \\
1055 \\
1075 \\
1090 \\
1237 \\
1365 \\
1400 \\
1275 \\
1134 \\
1107 \\
1187 \\
1155 \\
1128 \\
948 \\
925 \\
860 \\
860 \\
855 \\
840 \\
845 \\
803\end{array}$ \\
\hline $\begin{array}{l}9 \mathrm{n} \\
9 \mathrm{o} \\
9 \mathrm{p} \\
9 \mathrm{q} \\
9 \mathrm{r} \\
9 \mathrm{~s} \\
9 \mathrm{t} \\
9 \mathrm{u} \\
9 \mathrm{v} \\
9 \mathrm{w} \\
9 \mathrm{x} \\
9 \mathrm{y}\end{array}$ & $\begin{array}{ll}27^{\circ} & 5.59^{\prime} \\
27^{\circ} & 5.16^{\prime} \\
27^{\circ} & 4.84^{\prime} \\
27^{\circ} & 3.94^{\prime} \\
27^{\circ} & 3.54^{\prime} \\
27^{\circ} & 3.27^{\prime} \\
27^{\circ} & 2.81^{\prime} \\
27^{\circ} & 2.61^{\prime} \\
27^{\circ} & 2.19^{\prime} \\
27^{\circ} & 1.89^{\prime} \\
27^{\circ} & 1.40^{\prime} \\
27^{\circ} & 0.87^{\prime}\end{array}$ & 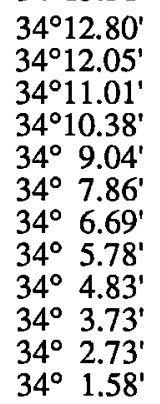 & $\begin{array}{r}137 \pm 17.5 \\
122 \pm 14.6 \\
150 \pm 19.0 \\
156 \pm 19.2 \\
170 \pm 21.1 \\
172 \pm 21.8 \\
80 \pm 14.6 \\
112 \pm 14.2 \\
103 \pm 12.8 \\
122 \pm 18.9 \\
101 \pm 17.1 \\
108 \pm 13.8\end{array}$ & $\begin{array}{l}1.09 \\
1.09 \\
1.10 \\
1.10 \\
1.11 \\
1.11 \\
1.12 \\
1.12 \\
1.13 \\
1.13 \\
1.14 \\
1.15\end{array}$ & $\begin{array}{r}126 \pm 2.2 \\
112 \pm 1.1 \\
136 \pm 2.4 \\
142 \pm 2.0 \\
153 \pm 2.5 \\
155 \pm 2.9 \\
71 \pm 5.4 \\
100 \pm 2.0 \\
91 \pm 1.7 \\
108 \pm 5.3 \\
89 \pm 5.6 \\
94 \pm 2.4\end{array}$ & $\begin{array}{l}750 \\
746 \\
770 \\
765 \\
970 \\
972 \\
737 \\
660 \\
546 \\
609 \\
447 \\
429\end{array}$ \\
\hline
\end{tabular}


TABLE 1b. Summary of Heat Flow Measurements: Central Line

\begin{tabular}{|c|c|c|c|c|c|c|}
\hline Station & $\begin{array}{l}\text { Latitude } \\
\text { N }\end{array}$ & $\underset{\mathrm{E}}{\text { Longitude }}$ & $\begin{array}{c}\text { Heat Flow, } \\
\mathrm{mW} \mathrm{m}^{-2}\end{array}$ & $\begin{array}{l}\text { Conductivity, } \\
\mathrm{W} \mathrm{m}^{-1} \mathrm{~K}^{-1}\end{array}$ & $\begin{array}{l}\text { Gradient, } \\
\mathbf{m}^{\circ} \mathrm{K} \mathrm{m}^{-1}\end{array}$ & $\begin{array}{l}\text { Water Depth, } \\
\text { m }\end{array}$ \\
\hline 1a & $27^{\circ} 14.45^{\prime}$ & $35^{\circ} 14.85^{\prime}$ & $144 \pm 18.0$ & 1.04 & $138 \pm 1.4$ & 681 \\
\hline $1 b$ & $27^{\circ} 12.83$ & $35^{\circ} 13.96^{\prime}$ & $114 \pm 16.7$ & 1.02 & $112 \pm 3.2$ & 687 \\
\hline $1 c$ & $27^{\circ} 13.19^{\prime}$ & $35^{\circ} 12.73^{\prime}$ & $121 \pm 15.3$ & 1.02 & $119 \pm 1.0$ & 690 \\
\hline $1 d$ & $27^{\circ} 12.57^{\prime}$ & $35^{\circ} 11.65^{\prime}$ & $149 \pm 19.8$ & 1.01 & $148 \pm 2.0$ & 700 \\
\hline $1 \mathrm{e}$ & $27^{\circ} 12.28^{\prime}$ & $35^{\circ} 9.96^{\prime}$ & $178 \pm 24.0$ & 1.00 & $178 \pm 2.6$ & 730 \\
\hline lf & $27^{\circ} 11.39^{\prime}$ & $35^{\circ} 8.31^{\prime}$ & $161 \pm 21.1$ & 0.99 & $163 \pm 1.6$ & 790 \\
\hline $1 \mathrm{~g}$ & $27^{\circ} 10.54^{\prime}$ & $35^{\circ} 7.14^{\prime}$ & $146 \pm 18.5$ & 0.98 & $149 \pm 0.6$ & 899 \\
\hline $1 \mathrm{~h}$ & $27^{\circ} 9.95^{\prime}$ & $35^{\circ} 5.76^{\prime}$ & $175 \pm 25.4$ & 0.97 & $180 \pm 3.9$ & 874 \\
\hline $1 \mathrm{i}$ & $27^{\circ} 9.50^{\prime}$ & $35^{\circ} 4.30^{\prime}$ & $167 \pm 21.8$ & 0.97 & $172 \pm 1.2$ & 925 \\
\hline $1 \mathrm{j}$ & $27^{\circ} 9.55^{\prime}$ & $35^{\circ} 3.05^{\prime}$ & $193 \pm 25.5$ & 0.96 & $201 \pm 1.4$ & 920 \\
\hline $1 \mathbf{k}$ & $27^{\circ} 9.65^{\prime}$ & $35^{\circ} 1.08^{\prime}$ & $183 \pm 23.8$ & 0.95 & $193 \pm 0.7$ & 960 \\
\hline 11 & $27^{\circ} 9.38^{\prime}$ & $34^{\circ} 59.72^{\prime}$ & $266 \pm 38.6$ & 0.95 & $280 \pm 5.3$ & 920 \\
\hline $1 \mathrm{~m}$ & $27^{\circ} 9.16^{\prime}$ & $34^{\circ} 58.83$ & $236 \pm 34.5$ & 0.94 & $251 \pm 4.7$ & 835 \\
\hline $2 a$ & $27^{\circ} 9.74^{\prime}$ & $34^{\circ} 56.99^{\prime}$ & $264 \pm 38.1$ & 0.94 & $281 \pm 4.7$ & 865 \\
\hline $2 b$ & $27^{\circ} 9.99^{\prime}$ & $34^{\circ} 56.43^{\prime}$ & $210 \pm 28.9$ & 0.93 & $226 \pm 1.9$ & 910 \\
\hline $2 c$ & $27^{\circ} 7.54^{\prime}$ & $34^{\circ} 56.34^{\prime}$ & $224 \pm 33.9$ & 0.92 & $243 \pm 5.1$ & 875 \\
\hline $2 d$ & $27^{\circ} 6.91^{\prime}$ & $34^{\circ} 55.56^{\prime}$ & $227 \pm 33.2$ & 0.92 & $247 \pm 3.9$ & 865 \\
\hline $2 \mathrm{e}$ & $27^{\circ} 6.86^{\prime}$ & $34^{\circ} 54.23^{\prime}$ & $204 \pm 30.6$ & 0.91 & $224 \pm 4.1$ & 840 \\
\hline $2 f$ & $27^{\circ} 5.07^{\prime}$ & $34^{\circ} 53.14^{\prime}$ & $181 \pm 27.7$ & 0.90 & $202 \pm 3.8$ & 890 \\
\hline $2 g$ & $27^{\circ} 4.71^{\prime}$ & $34^{\circ} 51.67^{\prime}$ & $191 \pm 29.3$ & 0.90 & $212 \pm 4.3$ & 930 \\
\hline $2 \mathrm{~h}$ & $27^{\circ} 4.00^{\prime}$ & $34^{\circ} 50.56^{\prime}$ & $221 \pm 32.8$ & 0.90 & $245 \pm 3.8$ & 1170 \\
\hline $2 \mathrm{i}$ & $27^{\circ} 3.67^{\prime}$ & $34^{\circ} 48.83^{\prime}$ & $256 \pm 40.0$ & 0.89 & $288 \pm 6.1$ & 1115 \\
\hline $2 \mathrm{j}$ & $27^{\circ} 2.87^{\prime}$ & $34^{\circ} 46.90^{\prime}$ & $220 \pm 33.6$ & 0.89 & $247 \pm 4.5$ & 1150 \\
\hline $2 \mathrm{k}$ & $27^{\circ} 3.05^{\prime}$ & $34^{\circ} 45.36^{\prime}$ & $247 \pm 35.9$ & 0.88 & $281 \pm 2.5$ & 1260 \\
\hline $3 a$ & $27^{\circ} 4.82^{\prime}$ & $34^{\circ} 44.03^{\prime}$ & $56 \pm 9.9$ & 0.88 & $64 \pm 2.5$ & 1431 \\
\hline $3 b$ & $27^{\circ} 4.43^{\prime}$ & $34^{\circ} 44.10^{\prime}$ & $615 \pm 94.1$ & 0.88 & $699 \pm 11.6$ & 1441 \\
\hline $3 c$ & $27^{\circ} 4.45^{\prime}$ & $34^{\circ} 44.33^{\prime}$ & $330 \pm 50.0$ & 0.88 & $375 \pm 5.7$ & 1441 \\
\hline $3 d$ & $27^{\circ} 4.43^{\prime}$ & $34^{\circ} 44.52^{\prime}$ & $189 \pm 27.2$ & 0.88 & $215 \pm 1.6$ & 1330 \\
\hline $3 \mathrm{e}$ & $27^{\circ} 1.66^{\prime}$ & $34^{\circ} 44.85^{\prime}$ & $309 \pm 43.5$ & 0.88 & $351 \pm 1.6$ & 1220 \\
\hline $3 \mathrm{f}$ & $27^{\circ} 0.77^{\prime}$ & $34^{\circ} 44.16^{\prime}$ & $312 \pm 44.7$ & 0.89 & $350 \pm 3.0$ & 1220 \\
\hline $3 g$ & $26^{\circ} 59.62^{\prime}$ & $34^{\circ} 43.59^{\prime}$ & $320 \pm 45.9$ & 0.89 & $360 \pm 3.0$ & 1236 \\
\hline $3 \mathrm{~h}$ & $26^{\circ} 59.40^{\prime}$ & $34^{\circ} 42.49^{\prime}$ & $252 \pm 35.4$ & 0.89 & $283 \pm 1.6$ & 1278 \\
\hline $3 \mathrm{i}$ & $26^{\circ} 57.75^{\prime}$ & $34^{\circ} 41.09^{\prime}$ & $111 \pm 15.6$ & 0.90 & $123 \pm 0.9$ & 1065 \\
\hline $3 j$ & $26^{\circ} 57.89^{\prime}$ & $34^{\circ} 39.86^{\prime}$ & $63 \pm 9.5$ & 0.90 & $70 \pm 1.2$ & 980 \\
\hline $3 \mathrm{k}$ & $26^{\circ} 56.86^{\prime}$ & $34^{\circ} 38.17^{\prime}$ & $190 \pm 35.5$ & 0.91 & $209 \pm 11.4$ & 935 \\
\hline 31 & $26^{\circ} 55.64^{\prime}$ & $34^{\circ} 36.47^{\prime}$ & $167 \pm 30.3$ & 0.92 & $182 \pm 9.2$ & 938 \\
\hline $3 \mathrm{~m}$ & $26^{\circ} 54.74^{\prime}$ & $34^{\circ} 43.90^{\prime}$ & $210 \pm 34.8$ & 0.92 & $228 \pm 8.1$ & 865 \\
\hline $4 a$ & $26^{\circ} 54.04^{\prime}$ & $34^{\circ} 32.69^{\prime}$ & $160 \pm 25.8$ & 0.93 & $172 \pm 5.5$ & 884 \\
\hline $4 b$ & $26^{\circ} 54.03^{\prime}$ & $34^{\circ} 30.87^{\prime}$ & $179 \pm 28.8$ & 0.94 & $190 \pm 6.4$ & 920 \\
\hline $4 c$ & $26^{\circ} 53.41^{\prime}$ & $34^{\circ} 29.51^{\prime}$ & $208 \pm 1.6$ & 0.94 & $221 \pm 5.4$ & 832 \\
\hline $4 d$ & $26^{\circ} 53.43^{\prime}$ & $34^{\circ} 28.24^{\prime}$ & $217 \pm 32.2$ & 0.95 & $228 \pm 5.1$ & 845 \\
\hline $4 e$ & $26^{\circ} 52.91^{\prime}$ & $34^{\circ} 26.72^{\prime}$ & $229 \pm 33.0$ & 0.96 & $239 \pm 4.5$ & 865 \\
\hline $4 f$ & $26^{\circ} 52.78^{\prime}$ & $34^{\circ} 25.30^{\prime}$ & $192 \pm 27.1$ & 0.96 & $200 \pm 3.2$ & 924 \\
\hline $4 \mathrm{~g}$ & $26^{\circ} 51.99^{\prime}$ & $34^{\circ} 23.84^{\prime}$ & $159 \pm 22.5$ & 0.97 & $164 \pm 2.9$ & 694 \\
\hline $4 h$ & $26^{\circ} 51.49^{\prime}$ & $34^{\circ} 22.83^{\prime}$ & $122 \pm 17.8$ & 0.98 & $124 \pm 3.0$ & 695 \\
\hline $4 i$ & $26^{\circ} 51.10^{\prime}$ & $34^{\circ} 21.77^{\prime}$ & $124 \pm 17.7$ & 0.99 & $125 \pm 2.7$ & 695 \\
\hline $4 j$ & $26^{\circ} 50.35^{\prime}$ & $34^{\circ} 21.19^{\prime}$ & $121 \pm 16.5$ & 1.00 & $121 \pm 2.0$ & 743 \\
\hline $4 \mathrm{k}$ & $26^{\circ} 49.53^{\prime}$ & $34^{\circ} 19.99^{\prime}$ & $124 \pm 19.0$ & 1.01 & $123 \pm 4.2$ & 743 \\
\hline 41 & $26^{\circ} 48.73^{\prime}$ & $34^{\circ} 19.07^{\prime}$ & $161 \pm 23.1$ & 1.02 & $158 \pm 4.1$ & 735 \\
\hline $4 \mathrm{~m}$ & $26^{\circ} 48.43^{\prime}$ & $34^{\circ} 18.18^{\prime}$ & $217 \pm 29.6$ & 1.03 & $211 \pm 4.2$ & 717 \\
\hline $4 n$ & $26^{\circ} 47.83^{\prime}$ & $34^{\circ} 17.25^{\prime}$ & $100 \pm 15.6$ & 1.04 & $96 \pm 3.9$ & 717 \\
\hline 40 & $26^{\circ} 47.14^{\prime}$ & $34^{\circ} 16.23^{\prime}$ & $131 \pm 16.6$ & 1.05 & $125 \pm 1.5$ & 825 \\
\hline $4 p$ & $26^{\circ} 46.76^{\prime}$ & $34^{\circ} 15.01^{\prime}$ & $88 \pm 15.5$ & 1.06 & $83 \pm 5.2$ & 711 \\
\hline $4 q$ & $26^{\circ} 46.43^{\prime}$ & $34^{\circ} 13.86^{\prime}$ & $181 \pm 23.1$ & 1.08 & $168 \pm 2.7$ & 679 \\
\hline $4 r$ & $26^{\circ} 46.25^{\prime}$ & $34^{\circ} 12.52^{\prime}$ & $106 \pm 13.9$ & 1.10 & $96 \pm 2.2$ & 654 \\
\hline $4 s$ & $26^{\circ} 46.05^{\prime}$ & $34^{\circ} 11.39^{\prime}$ & $111 \pm 16.0$ & 1.11 & $100 \pm 3.6$ & 681 \\
\hline $4 \mathrm{t}$ & $26^{\circ} 45.52^{\prime}$ & $34^{\circ} 9.86^{\prime}$ & $111 \pm 15.9$ & 1.13 & $98 \pm 3.7$ & 666 \\
\hline $4 u$ & $26^{\circ} 45.32^{\prime}$ & $34^{\circ} 8.77^{\prime}$ & $120 \pm 16.4$ & 1.14 & $105 \pm 3.3$ & 636 \\
\hline
\end{tabular}


TABLE 1c. Summary of Heat Flow Measurements: Southern Line

\begin{tabular}{|c|c|c|c|c|c|c|}
\hline Station & $\begin{array}{l}\text { Latitude } \\
\qquad \mathbf{N}\end{array}$ & $\begin{array}{l}\text { Longitude } \\
\text { E }\end{array}$ & $\begin{array}{l}\text { Heat Flow, } \\
\mathrm{mW} \mathrm{m}^{-2}\end{array}$ & $\begin{array}{l}\text { Conductivity, } \\
\mathrm{W} \mathrm{m}^{-1}{ }^{\circ} \mathrm{K}^{-1}\end{array}$ & $\begin{array}{l}\text { Gradient, } \\
\mathrm{m}^{\circ} \mathrm{K} \mathrm{m}^{-1}\end{array}$ & $\begin{array}{l}\text { Water Depth, } \\
\text { m }\end{array}$ \\
\hline $10 \mathrm{a}$ & $26^{\circ} 55.73^{\prime}$ & $35^{\circ} 35.29^{\prime}$ & $122 \pm 15.9$ & 1.04 & $117 \pm 1.8$ & 855 \\
\hline $10 \mathrm{~b}$ & $26^{\circ} 54.70^{\prime}$ & $35^{\circ} 34.63^{\prime}$ & $90 \pm 11.8$ & 1.04 & $87 \pm 1.3$ & 561 \\
\hline $10 c$ & $26^{\circ} 54.60^{\prime}$ & $35^{\circ} 34.38^{\prime}$ & $96 \pm 13.2$ & 1.03 & $93 \pm 2.0$ & 600 \\
\hline $10 d$ & $26^{\circ} 54.15^{\prime}$ & $35^{\circ} 32.75^{\prime}$ & $126 \pm 16.6$ & 1.03 & $122 \pm 1.9$ & 567 \\
\hline $10 \mathrm{e}$ & $26^{\circ} 53.68^{\prime}$ & $35^{\circ} 32.01^{\prime}$ & $165 \pm 21.9$ & 1.02 & $162 \pm 2.4$ & 510 \\
\hline $10 f$ & $26^{\circ} 53.31^{\prime}$ & $35^{\circ} 31.05^{\prime}$ & $210 \pm 26.1$ & 1.02 & $206 \pm 1.4$ & 522 \\
\hline $10 \mathrm{~g}$ & $26^{\circ} 53.25^{\prime}$ & $35^{\circ} 30.12^{\prime}$ & $146 \pm 20.2$ & 1.01 & $145 \pm 2.8$ & 507 \\
\hline $10 \mathrm{~h}$ & $26^{\circ} 53.26^{\prime}$ & $35^{\circ} 28.94^{\prime}$ & $293 \pm 41.6$ & 1.01 & $290 \pm 6.7$ & 560 \\
\hline $10 \mathrm{i}$ & $26^{\circ} 52.76^{\prime}$ & $35^{\circ} 28.92^{\prime}$ & $158 \pm 21.3$ & 1.00 & $158 \pm 2.3$ & 560 \\
\hline $10 \mathrm{j}$ & $26^{\circ} 51.93^{\prime}$ & $35^{\circ} 28.01^{\prime}$ & $119 \pm 18.2$ & 1.00 & $119 \pm 3.9$ & 574 \\
\hline $10 \mathrm{k}$ & $26^{\circ} 51.49^{\prime}$ & $35^{\circ} 26.83^{\prime}$ & $100 \pm 14.1$ & 0.99 & $101 \pm 2.0$ & 585 \\
\hline 101 & $26^{\circ} 51.16^{\prime}$ & $35^{\circ} 25.77^{\prime}$ & $111 \pm 15.4$ & 0.98 & $113 \pm 1.9$ & 588 \\
\hline $10 \mathrm{~m}$ & $26^{\circ} 50.63^{\prime}$ & $35^{\circ} 24.66^{\prime}$ & $114 \pm 14.8$ & 0.98 & $116 \pm 0.9$ & 623 \\
\hline $10 n$ & $26^{\circ} 50.31^{\prime}$ & $35^{\circ} 23.65^{\prime}$ & $121 \pm 17.5$ & 0.97 & $125 \pm 2.6$ & 685 \\
\hline 100 & $26^{\circ} 49.92^{\prime}$ & $35^{\circ} 22.91^{\prime}$ & $148 \pm 20.7$ & 0.96 & $154 \pm 2.3$ & 700 \\
\hline $10 p$ & $26^{\circ} 49.32^{\prime}$ & $35^{\circ} 21.50^{\prime}$ & $142 \pm 21.2$ & 0.96 & $148 \pm 3.6$ & 714 \\
\hline $10 \mathrm{q}$ & $26^{\circ} 49.10^{\prime}$ & $35^{\circ} 19.89^{\prime}$ & $164 \pm 22.8$ & 0.95 & $173 \pm 2.1$ & 735 \\
\hline $10 \mathrm{r}$ & $26^{\circ} 48.06^{\prime}$ & $35^{\circ} 18.93^{\prime}$ & $195 \pm 27.8$ & 0.94 & $207 \pm 3.1$ & 778 \\
\hline $10 \mathrm{~s}$ & $26^{\circ} 47.25^{\prime}$ & $35^{\circ} 17.74^{\prime}$ & $149 \pm 20.9$ & 0.93 & $160 \pm 1.8$ & 763 \\
\hline $10 t$ & $26^{\circ} 47.06^{\prime}$ & $35^{\circ} 16.08^{\prime}$ & - & - & - & 923 \\
\hline $10 u$ & $26^{\circ} 47.06^{\prime}$ & $35^{\circ} 16.05^{\prime}$ & $153 \pm 26.7$ & 0.92 & $167 \pm 7.2$ & 923 \\
\hline $10 \mathrm{v}$ & $26^{\circ} 46.85^{\prime}$ & $35^{\circ} 14.99^{\prime}$ & $222 \pm 34.6$ & 0.91 & $244 \pm 5.8$ & 957 \\
\hline $10 w$ & $26^{\circ} 46.11^{\prime}$ & $35^{\circ} 14.00^{\prime}$ & $209 \pm 33.0$ & 0.91 & $230 \pm 5.9$ & 952 \\
\hline $10 x$ & $26^{\circ} 45.57^{\prime}$ & $35^{\circ} 12.71^{\prime}$ & $218 \pm 35.0$ & 0.90 & $242 \pm 6.6$ & 952 \\
\hline $10 y$ & $26^{\circ} 45.30^{\prime}$ & $35^{\circ} 11.79^{\prime}$ & $215 \pm 34.0$ & 0.90 & $239 \pm 5.9$ & 985 \\
\hline $10 \mathrm{z}$ & $26^{\circ} 44.93^{\prime}$ & $35^{\circ} 11.00^{\prime}$ & $203 \pm 31.3$ & 0.89 & $228 \pm 4.4$ & 1018 \\
\hline 10aa & $26^{\circ} 44.54^{\prime}$ & $35^{\circ} 9.88^{\prime}$ & - & - & - & 1031 \\
\hline $11 a$ & $26^{\circ} 44.21^{\prime}$ & $35^{\circ} 10.16^{\prime}$ & $218 \pm 34.3$ & 0.88 & $248 \pm 5.2$ & 1030 \\
\hline $11 \mathrm{~b}$ & $26^{\circ} 43.94^{\prime}$ & $35^{\circ} 8.88^{\prime}$ & $203 \pm 32.2$ & 0.88 & $231 \pm 5.1$ & 1030 \\
\hline $11 \mathrm{c}$ & $26^{\circ} 43.02^{\prime}$ & $35^{\circ} 7.54^{\prime}$ & $188 \pm 29.9$ & 0.87 & $216 \pm 4.6$ & 1090 \\
\hline $11 d$ & $26^{\circ} 42.35^{\prime}$ & $35^{\circ} 6.18^{\prime}$ & $172 \pm 28.0$ & 0.86 & $200 \pm 4.6$ & 1091 \\
\hline $11 \mathrm{e}$ & $26^{\circ} 42.26^{\prime}$ & $35^{\circ} 5.11^{\prime}$ & $171 \pm 27.9$ & 0.85 & $201 \pm 4.5$ & 1091 \\
\hline $11 \mathrm{f}$ & $26^{\circ} 41.71^{\prime}$ & $35^{\circ} 3.94^{\prime}$ & $264 \pm 44.2$ & 0.85 & $311 \pm 8.1$ & 1147 \\
\hline $11 \mathrm{~g}$ & $26^{\circ} 41.17^{\prime}$ & $35^{\circ} 2.80^{\prime}$ & $280 \pm 44.8$ & 0.86 & $326 \pm 6.6$ & 1160 \\
\hline $11 \mathrm{~h}$ & $26^{\circ} 41.14^{\prime}$ & $35^{\circ} 2.50^{\prime}$ & $223 \pm 36.4$ & 0.86 & $259 \pm 6.2$ & 1165 \\
\hline $11 \mathrm{i}$ & $26^{\circ} 40.18^{\prime}$ & $34^{\circ} 59.43^{\prime}$ & $291 \pm 46.0$ & 0.87 & $335 \pm 6.7$ & 1165 \\
\hline $11 j$ & $26^{\circ} 39.32^{\prime}$ & $34^{\circ} 58.08^{\prime}$ & $238 \pm 37.9$ & 0.88 & $271 \pm 6.1$ & 1207 \\
\hline $11 \mathrm{k}$ & $26^{\circ} 39.23^{\prime}$ & $34^{\circ} 57.00^{\prime}$ & $242 \pm 39.7$ & 0.88 & $275 \pm 7.6$ & 1205 \\
\hline 111 & $26^{\circ} 38.74^{\prime}$ & $34^{\circ} 55.99^{\prime}$ & $235 \pm 35.6$ & 0.89 & $264 \pm 4.4$ & 1250 \\
\hline $11 \mathrm{~m}$ & $26^{\circ} 38.26^{\prime}$ & $34^{\circ} 54.98^{\prime}$ & $242 \pm 36.9$ & 0.89 & $272 \pm 4.8$ & 1180 \\
\hline $11 n$ & $26^{\circ} 38.14^{\prime}$ & $34^{\circ} 53.83^{\prime}$ & $229 \pm 37.7$ & 0.90 & $254 \pm 8.0$ & 965 \\
\hline 110 & $26^{\circ} 38.30^{\prime}$ & $34^{\circ} 53.71^{\prime}$ & $230 \pm 37.4$ & 0.90 & $255 \pm 7.6$ & 945 \\
\hline $11 p$ & $26^{\circ} 36.67^{\prime}$ & $34^{\circ} 52.99^{\prime}$ & $256 \pm 39.8$ & 0.90 & $284 \pm 6.3$ & 948 \\
\hline $11 \mathrm{q}$ & $26^{\circ} 36.57^{\prime}$ & $34^{\circ} 51.58^{\prime}$ & $155 \pm 2.3$ & 0.90 & $172 \pm 1.8$ & 922 \\
\hline $11 \mathrm{r}$ & $26^{\circ} 36.37^{\prime}$ & $34^{\circ} 50.45^{\prime}$ & $210 \pm 35.1$ & 0.90 & $233 \pm 7.9$ & 960 \\
\hline $11 \mathrm{~s}$ & $26^{\circ} 36.10^{\prime}$ & $34^{\circ} 49.50^{\prime}$ & $194 \pm 29.6$ & 0.90 & $216 \pm 4.1$ & 972 \\
\hline $11 \mathrm{t}$ & $26^{\circ} 35.25^{\prime}$ & $34^{\circ} 48.40^{\prime}$ & $201 \pm 28.7$ & 0.90 & $223 \pm 2.1$ & 930 \\
\hline $12 a$ & $26^{\circ} 34.65^{\prime}$ & $34^{\circ} 47.54^{\prime}$ & $192 \pm 27.7$ & 0.91 & $211 \pm 2.6$ & 972 \\
\hline $12 \mathrm{~b}$ & $26^{\circ} 34.08^{\prime}$ & $34^{\circ} 46.53^{\prime}$ & $167 \pm 28.2$ & 0.91 & $183 \pm 6.9$ & 928 \\
\hline $12 c$ & $26^{\circ} 33.67^{\circ}$ & $34^{\circ} 45.44^{\prime}$ & $140 \pm 22.0$ & 0.91 & $154 \pm 3.9$ & 885 \\
\hline $12 d$ & $26^{\circ} 33.15^{\prime}$ & $34^{\circ} 44.30^{\prime}$ & $175 \pm 27.2$ & 0.91 & $192 \pm 4.6$ & 962 \\
\hline $12 e$ & $26^{\circ} 32.57^{\prime}$ & $34^{\circ} 43.09^{\prime}$ & $174 \pm 25.6$ & 0.91 & $191 \pm 2.9$ & 815 \\
\hline $12 \mathrm{f}$ & $26^{\circ} 32.22^{\prime}$ & $34^{\circ} 41.68^{\prime}$ & $192 \pm 34.0$ & 0.91 & $211 \pm 9.5$ & 830 \\
\hline $12 \mathrm{~g}$ & $26^{\circ} 31.84^{\prime}$ & $34^{\circ} 40.63^{\prime}$ & $168 \pm 26.4$ & 0.91 & $185 \pm 4.6$ & 820 \\
\hline $12 \mathrm{~h}$ & $26^{\circ} 31.49^{\prime}$ & $34^{\circ} 39.53^{\prime}$ & $-\quad-$ & - & & 844 \\
\hline $12 \mathrm{i}$ & $26^{\circ} 31.62^{\prime}$ & $34^{\circ} 39.28^{\prime}$ & $178 \pm 30.5$ & 0.91 & $196 \pm 7.7$ & 844 \\
\hline $12 j$ & $26^{\circ} 30.52^{\prime}$ & $34^{\circ} 37.78^{\prime}$ & - & - & - & 762 \\
\hline $12 \mathrm{k}$ & $26^{\circ} 30.47^{\prime}$ & $34^{\circ} 37.53$ & $205 \pm 31.5$ & 0.92 & $223 \pm 5.1$ & 896 \\
\hline 121 & $26^{\circ} 29.76^{\prime}$ & $34^{\circ} 35.53^{\prime}$ & $98 \pm 15.1$ & 0.92 & $107 \pm 2.5$ & 808 \\
\hline $12 \mathrm{~m}$ & $26^{\circ} 29.20^{\prime}$ & $34^{\circ} 34.07^{\prime}$ & $136 \pm 22.0$ & 0.93 & $146 \pm 4.8$ & 980 \\
\hline $12 n$ & $26^{\circ} 28.24^{\prime}$ & $34^{\circ} 32.24^{\prime}$ & $142 \pm 20.0$ & 0.95 & $149 \pm 2.2$ & 875 \\
\hline $13 a$ & $26^{\circ} 28.00^{\prime}$ & $34^{\circ} 31.43^{\prime}$ & $133 \pm 19.8$ & 0.96 & $139 \pm 3.3$ & 750 \\
\hline $13 \mathrm{~b}$ & $26^{\circ} 27.71^{\prime}$ & $34^{\circ} 30.21^{\prime}$ & $212 \pm 34.0$ & 0.97 & $219 \pm 8.0$ & 780 \\
\hline $13 c$ & $26^{\circ} 27.84^{\prime}$ & $34^{\circ} 29.36^{\prime}$ & $225 \pm 39.9$ & 0.98 & $230 \pm 12.6$ & 767 \\
\hline $13 d$ & $26^{\circ} 27.02^{\prime}$ & $34^{\circ} 28.68^{\prime}$ & $145 \pm 20.4$ & 0.99 & $146 \pm 2.9$ & 810 \\
\hline $13 e$ & $26^{\circ} 26.36^{\prime}$ & $34^{\circ} 27.88^{\prime}$ & $132 \pm 17.6$ & 1.00 & $132 \pm 1.8$ & 814 \\
\hline $13 \mathrm{f}$ & $26^{\circ} 26.03^{\prime}$ & $34^{\circ} 26.59^{\prime}$ & $126 \pm 17.9$ & 1.01 & $125 \pm 2.9$ & 784 \\
\hline
\end{tabular}


TABLE 1c. (continued)

\begin{tabular}{|c|c|c|c|c|c|c|}
\hline Station & $\begin{array}{l}\text { Latitude } \\
\mathrm{N}\end{array}$ & $\begin{array}{l}\text { Longitude } \\
\text { E }\end{array}$ & $\begin{array}{l}\text { Heat Flow, } \\
\mathrm{mW} \mathrm{m}^{-2}\end{array}$ & $\begin{array}{l}\text { Conductivity, } \\
\mathrm{W} \mathrm{m} \mathrm{m}^{-1}{ }^{\circ} \mathrm{K}^{-1}\end{array}$ & $\begin{array}{l}\text { Gradient, } \\
\mathbf{m}^{\circ} \mathrm{K} \mathbf{~ m}^{-1}\end{array}$ & $\begin{array}{c}\text { Water Depth } \\
\text { m }\end{array}$ \\
\hline $\begin{array}{l}13 \mathrm{~g} \\
13 \mathrm{~h} \\
13 \mathrm{i} \\
13 \mathrm{j} \\
13 \mathrm{k} \\
131\end{array}$ & $\begin{array}{l}26^{\circ} 25.44^{\prime} \\
26^{\circ} 25.06^{\prime} \\
26^{\circ} 24.36^{\prime} \\
26^{\circ} 23.81^{\prime} \\
26^{\circ} 23.19^{\prime} \\
26^{\circ} 22.75^{\prime}\end{array}$ & $\begin{array}{l}34^{\circ} 25.47^{\prime} \\
34^{\circ} 24.28 \\
34^{\circ} 23.16^{\prime} \\
34^{\circ} 21.86^{\prime} \\
34^{\circ} 20.83^{\prime} \\
34^{\circ} 19.63^{\prime}\end{array}$ & $\begin{array}{r}148 \pm 18.2 \\
155 \pm 21.3 \\
172 \pm 24.0 \\
122 \pm 20.3 \\
104 \pm 13.1 \\
69 \pm 10.5\end{array}$ & $\begin{array}{l}1.03 \\
1.04 \\
1.06 \\
1.08 \\
1.09 \\
1.11\end{array}$ & $\begin{array}{r}144 \pm 0.9 \\
149 \pm 3.3 \\
162 \pm 4.3 \\
115 \pm 6.0 \\
95 \pm 1.6 \\
62 \pm 2.8\end{array}$ & $\begin{array}{l}812 \\
813 \\
815 \\
699 \\
538 \\
425\end{array}$ \\
\hline
\end{tabular}

measured on board ship soon after recovery. The measurements were made by the needle probe method [von Herzen and Maxwell, 1959] at 0.2-m intervals along the length of each core. The measured thermal conductivities are shown plotted against distance from the top of each core in Figure 3. Systematic variations with depth which are present in all of the cores appear to correlate with climatically induced changes in planktonic microfaunnal and mineralogic composition [Herman and Rosenberg, 1969]. The individual conductivity measurements were corrected to in situ temperature and pressure values following the formula of Ratcliffe [1960] and the harmonic mean of these measurements was the value adopted for each core as given in Table 2 .

Two related trends exist in the spatial distribution of thermal conductivities in the Red Sea. The conductivities decrease with increasing water depth and with increasing distance from the coasts. Both of these trends are probably related to an increasing component of higher conductivity terrigenous sediments in the calcareous bottom muds with proximity to the coasts. The systematic spatial variation in conductivity was contoured as shown in Figure 2. This contour map was used to directly assign conductivities to the heat flow stations based on their map position. The heat flow values obtained are given in Tables $1 \mathrm{a}, 1 \mathrm{~b}$, and $1 \mathrm{c}$ for the northern, central, and southern transects respectively.

\section{Assessment of Heat Flow Data}

Heat flow measurements may be affected by environmental factors which complicate their interpretation and which need to be taken into account. Among these are seasonal and climatic temperature changes which create thermal transients, thermal refraction caused by relief on the seafloor and underlaying layers of contrasting conductivity, variations in thermal conductivity, sedimentation, and hydrothermal circulation. Because of the large number of heat flow measurements and their nearly uniform distribution along each transect across the rift, random point-to-point variations will tend to appear as scatter about the large-scale trend. A more important concern is the possible presence of systematic variations which might affect the overall trend of the heat flow from the shallower marginal areas to the deeper axial depression. We attempt to assess the influence of the environmental factors mentioned above in terms of rift-wide systematic effects that may be present.

\section{Seasonal and Climatic Temperature Variations}

Variations in water temperature with depth for the northern Red Sea from Wyrtki [1971] are shown in Figure 4. The horizontal bars indicate the range of recorded variation in temperature at various depths. Although water depths in the northern Red Sea are relatively shallow, recorded temperature variations are confined to the upper $300 \mathrm{~m}$ and thus do not significantly affect our bottom temperature gradient measurements all of which were made in water depths greater than 400 $m$. No corrections were therefore necessary for seasonal thermal transients.

Longer time scale climatic changes can alter the average seafloor temperature and potentially result in anomalous temperature gradients. The effect of climatic temperature variations depends on the time since the temperature change, on its duration, and on its magnitude. Long-term seafloor temperature changes that may have significant effects on the measured gradients reported here are assumed to result from two possible causes. The first is the relatively cooler period associated with the most recent glacial age. The second is the relatively warmer period before the transition to a marine environment at the end of the Miocene; a time when the inferred prevailing condition under which the evaporites were deposited was that of a shallow water/sabkah environment.

Estimates of the temperature history of the Red Sea nearsurface waters for the most recent glacial period have been obtained from studies of the variation in relative abundance of planktonic microfossil groups found in sediment cores [Wall and Warren, 1969; Berggren and Boersma, 1969; Berggren, 1969]. These studies indicate that the temperature of the nearsurface waters of the Red Sea has been relatively constant for the last 11,000 years. Between 11,000 and 70,000 years ago

TABLE 2. Thermal Conductivity Measurements

\begin{tabular}{|c|c|c|c|c|c|c|}
\hline Core & $\begin{array}{l}\text { Latitude } \\
\quad \mathrm{N}\end{array}$ & $\begin{array}{l}\text { Longitude } \\
\text { E }\end{array}$ & $\begin{array}{l}\text { Conductivity, } \\
\mathrm{W} \mathrm{m}^{-1}{ }^{\circ} \mathrm{K}^{-1}\end{array}$ & $\begin{array}{l}\text { Standard Deviation, } \\
\quad \mathrm{W} \mathbf{m}^{-1}{ }^{\circ} \mathrm{K}^{-1}\end{array}$ & $\begin{array}{l}\text { Length, } \\
\text { m }\end{array}$ & $\begin{array}{c}\text { Water Depth, } \\
\text { m }\end{array}$ \\
\hline $\begin{array}{l}\mathrm{C} 1 \\
\mathrm{C} 2 \\
\mathrm{C} 3 \\
\mathrm{C} 4 \\
\mathrm{C} 5 \\
\mathrm{C} 6 \\
\mathrm{C} 7 \\
\mathrm{C} 8 \\
\mathrm{C} 9 \\
\mathrm{C} 10\end{array}$ & $\begin{array}{rr}27^{\circ} & 2.87^{\prime} \\
26^{\circ} & 46.01^{\prime} \\
27^{\circ} & 15.14^{\prime} \\
27^{\circ} & 8.44^{\prime} \\
27^{\circ} & 6.79^{\prime} \\
27^{\circ} & 12.72^{\prime} \\
27^{\circ} & 17.92^{\prime} \\
26^{\circ} & 28.85^{\prime} \\
26^{\circ} & 37.26^{\prime} \\
26^{\circ} & 42.42^{\prime}\end{array}$ & $\begin{array}{lr}34^{\circ} & 44.53^{\prime} \\
34^{\circ} & 9.79^{\prime} \\
35^{\circ} & 15.11^{\prime} \\
35^{\circ} & 0.09^{\prime} \\
34^{\circ} & 14.36^{\prime} \\
34^{\circ} & 26.66^{\prime} \\
34^{\circ} & 38.66^{\prime} \\
34^{\circ} & 34.84^{\prime} \\
34^{\circ} & 52.57^{\prime} \\
35^{\circ} & 5.15^{\prime}\end{array}$ & $\begin{array}{l}0.88 \\
1.13 \\
1.04 \\
0.95 \\
1.08 \\
1.02 \\
1.01 \\
0.92 \\
0.90 \\
0.85\end{array}$ & $\begin{array}{l}0.10 \\
0.12 \\
0.08 \\
0.08 \\
0.12 \\
0.09 \\
0.09 \\
0.11 \\
0.10 \\
0.11\end{array}$ & $\begin{array}{l}4.6 \\
5.6 \\
5.0 \\
5.2 \\
5.2 \\
5.6 \\
5.5 \\
6.0 \\
5.4 \\
5.7\end{array}$ & $\begin{array}{r}1313 \\
709 \\
732 \\
996 \\
906 \\
1194 \\
1147 \\
975 \\
1040 \\
1249\end{array}$ \\
\hline
\end{tabular}



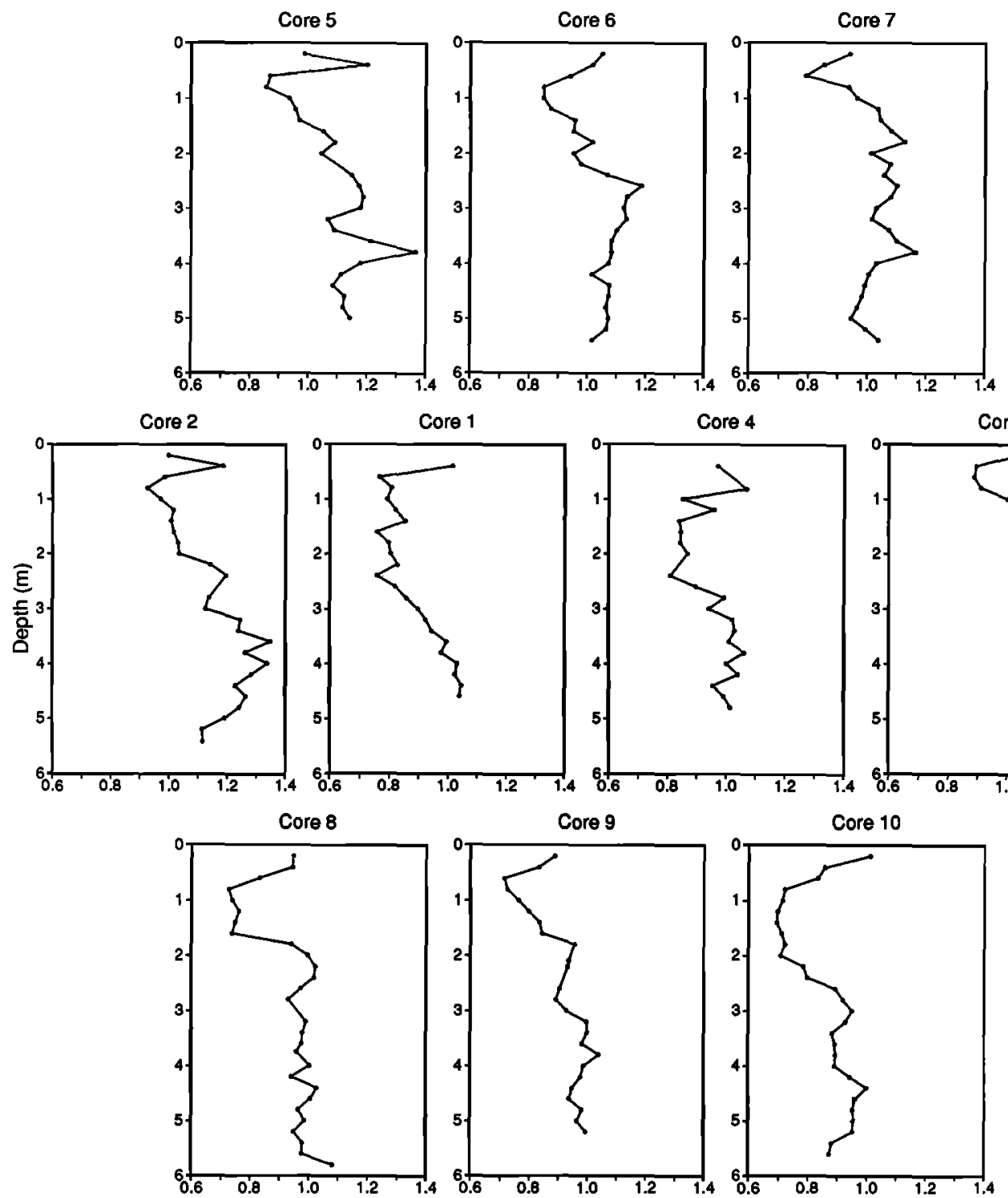

Core $1 \quad$ Core 4
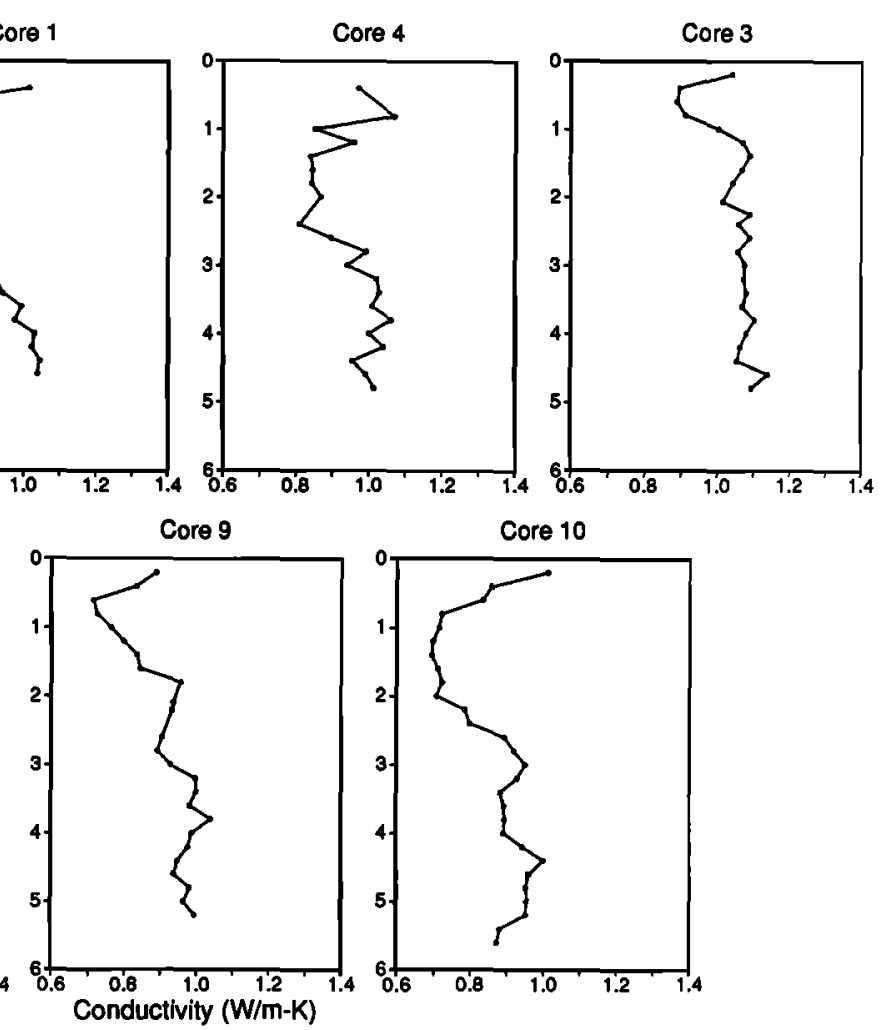

Fig. 3. Plots of thermal conductivity against depth from the top of each core. Plots are arranged according to their relative position on the heat flow transects (see Figure 2).

a series of relatively cooler periods prevailed. A simplified step function temperature history derived from the work of Wall and Warren [1969] and the review of Berggren [1969] is given in Table 3. We assume that near-surface water temperature changes coincide with equal seafloor temperature changes on the time scales considered here. In addition, we assume $\mathrm{a}+15^{\circ} \mathrm{C}$ temperature change relative to present-day seafloor temperatures for pre-Pliocene time, before marine conditions became established in the Red Sea. We model the seafloor thermal gradient anomaly resulting from this temperature history using the method described by Birch [1948] for an infinite half-space undergoing stepwise temperature changes at its surface. The results are given in Table 3 . The main perturbation to the seafloor thermal gradients is caused by the cooling associated with the glacial period between 11,000 and 70,000 years ago. The lower temperatures in this period may account for a gradient disturbance of $-4.6 \mathrm{~m}^{\circ} \mathrm{K} / \mathrm{m}$ at the seafloor. The effect of the pre-Pliocene warm period is far less significant, accounting for only a $+0.6 \mathrm{~m}^{\circ} \mathrm{K} / \mathrm{m}$ disturbance, despite the fact that it is modeled here as extending indefinitely into the past. The total temperature gradient distur- bance is about $-4 \mathrm{~m}^{\circ} \mathrm{K} / \mathrm{m}$ or roughly $2 \%$ of the average of the measured gradients. The microfossil studies cited above suggest that there may have been shorter-period temperature fluctuations within the simplified temperature steps modeled here. This would result in an even smaller calculated gradient disturbance. We thus consider corrections to the temperature gradient measurements due to climatic temperature changes negligible. This conclusion is also supported by the evaluation of Erickson et al. [1975] of geothermal measurements made in the Red Sea by the Deep Sea Drilling Project (DSDP), which measured temperatures over much greater depth intervals than those of our probe. They find no convincing evidence for climatically induced heat flow variations with depth.

\section{Topographic Effects}

The seafloor topography of the northern Red Sea is relatively rough having relief of one to two hundred meters over a few kilometers distance. In addition, a thick layer of middle to late Miocene evaporites exists a few hundred meters below the seafloor and in many places is deformed into diapirs which 


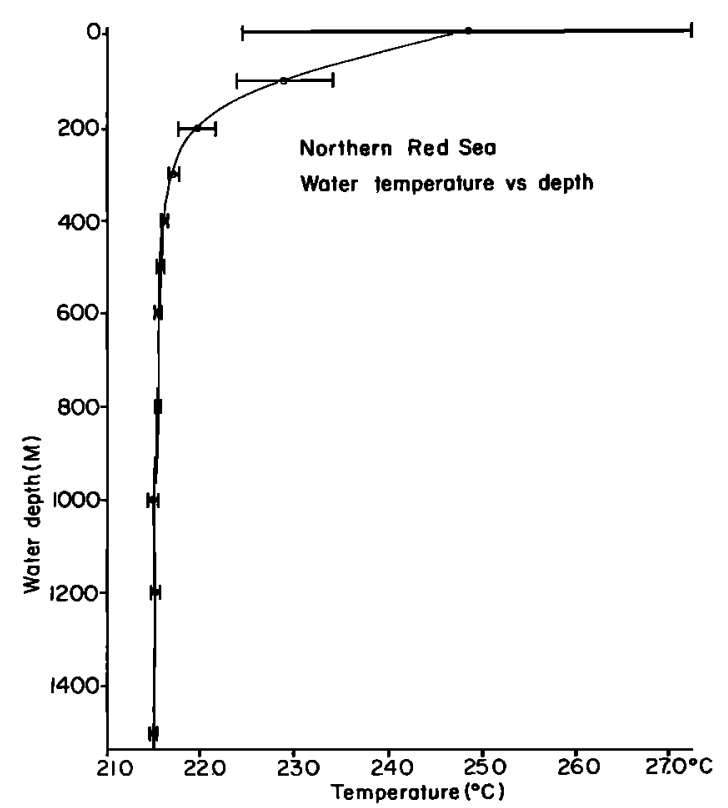

Fig. 4. Plot of recorded temperature variations with water depth for the Red Sea. Horizontal bars indicate the range of recorded variations. Below about $300 \mathrm{~m}$, the water temperatures are stable. Data are from Wyrtki [1971].

also have substantial relief. The Red Sea evaporites have been sampled to the south by drilling and have been found to have conductivies about 5 times greater than the overlaying Pliocene sediments [Whieldon et al., 1974; Girdler et al., 1974]. Preliminary modeling of the refraction effect from these layers suggested that this effect could account for the small-scale variations in measured heat flow [Hobart et al., 1985]. The effect of the seafloor topography and relief on the evaporites was assessed by means of a numerical two-dimensional steady state finite difference calculation using the relaxation method. Seafloor and top of evaporite relief was digitized from water gun single-channel seismic profiles which were nearly coincident with the heat flow transects. In addition, an oil industry air gun multichannel seismic line, ERS-74 [Barakat and Miller, 1984; Miller and Barakat, 1988], roughly coincident with the western half of our central heat flow transect (see Figure 2 for location) was digitized for these reflectors and for the bottom of the evaporite layer which was resolved in many places on the multichannel line, but which we were unable to resolve on our single-channel lines. Constant thermal conductivities were assigned to each of the layers, and a constant heat flow condition was imposed at the bottom boundary of the grid. In this calculation, a value of $1.1 \mathrm{~W} \mathrm{~m}^{-1}{ }^{\circ} \mathrm{K}^{-1}$ was adopted for the post-evaporite sediment conductivity which is near the upper end of our measured conductivities, but more within the range measured in the southern Red Sea at DSDP sites 225, 227, and 228 [Girdler et al., 1974] which sample a much greater sediment thickness. For the evaporites, a value of $5.1 \mathrm{~W} \mathrm{~m}^{-1}$ ${ }^{\circ} \mathrm{K}^{-1}$ was adopted which is within the range of values for anhydrite and halite [Touloukian and Ho, 1981], the main constituents of the evaporites, and is also within the range measured at DSDP sites in the southern Red Sea [Wheildon et al., 1974]. An assumed conductivity of $3.1 \mathrm{~W} \mathrm{~m}^{-1}{ }^{\circ} \mathrm{K}^{-1}$ was adopted for the pre-evaporite layer which is a typical nominal value for continental basement rocks but is probably a bit high for the pre-evaporite clastics.

Results of this calculation for the multichannel line including the effects of the bottom of the evaporites are shown in Figure 5. No significant systematic effect is generated by the overall thickening of the evaporites toward the center of the rift or by the overall thinning of the post-evaporite layer. Most of the relative heat flow variation is scattered within $20 \%$ of the bottom heat input. Similar results were obtained for the northern and southern lines, although for these cases only the seafloor and the top of the evaporites were modeled. The effect of including the bottom boundary of the evaporites differed by less than $2 \%$ from not including it due to the high conductivity and great thickness ( $2 \mathrm{~km}$ on average) of the evaporite layer and the smaller conductivity contrast between the evaporite and pre-evaporite layer.

An attempt was made to use the calculated heat flow disturbance to correct individual heat flow values, but this proved unsuccessful in reducing the scatter. The major reason for this is the uncertainty in locating the precise projected position of the measurement with respect to the bottom relief on the digitized seismic profiles. Another reason is that the seismic profiles only record apparent slopes in the direction of the profiles and although these were made perpendicular to the main trend of the rift, many local variations in trend exist. The fact that the observed point-to-point scatter in the heat flow values is generally about the same magnitude as that predicted from the numerical calculation suggests, however, that relief on the seafloor and top of the evaporites on can adequately account for most of the local scatter.

\section{Thermal Conductivities}

Thermal conductivity variations observed in the sediment piston cores have a total range of $0.85-1.13 \mathrm{~W} \mathrm{~m}^{-1}{ }^{\circ} \mathrm{K}^{-1}$, a $28 \%$ variation. However, the mapped spatial distribution of conductivity in the Red Sea suggests a gradual transition from higher values near the coasts to lower values near the deeper central axis (see Figure 2 and Table 2). The spatial variation appears to be systematic and can be smoothly contoured as shown in Figure 2. It appears that the decrease in the conductivity toward the center of the sea is due to a smaller amount of higher-conductivity terrigenous component in the sediment but may also be due to a systematic variation in grain size affecting the sediment porosity. Although not precisely defined with the available measurements, the contoured thermal conductivities suggest a slightly asymmetric distribution within the rift. The northeastern portion of the contoured area appears to form a broader region of higher thermal conductivity. This suggests that a significant component of higher-conductivity terrigenous

TABLE 3. Climatic Gradient Variations

\begin{tabular}{cllcc}
\hline Period & $\begin{array}{l}\text { End Time, } \\
\text { years B.P. }\end{array}$ & $\begin{array}{l}\text { Start time, } \\
\text { years B.P. }\end{array}$ & $\begin{array}{c}\Delta \mathrm{T}, \\
{ }^{\circ} \mathrm{C}\end{array}$ & $\begin{array}{c}\Delta \mathrm{Q}, \\
\mathrm{m}^{\circ} \mathrm{K} / \mathrm{m}\end{array}$ \\
\hline 1 & 11,000 & 13,000 & -5 & -0.4 \\
2 & 13,000 & 23,000 & -10 & -2.2 \\
3 & 23,000 & 60,000 & -7 & -1.8 \\
4 & 60,000 & 70,000 & -5 & -0.2 \\
5 & $5 \times 10^{6}$ & $\infty$ & +15 & +0.6 \\
\hline
\end{tabular}

Thermal diffusivity is $10^{-6} \mathrm{~m}^{2} \mathrm{~s}^{-1}$. 

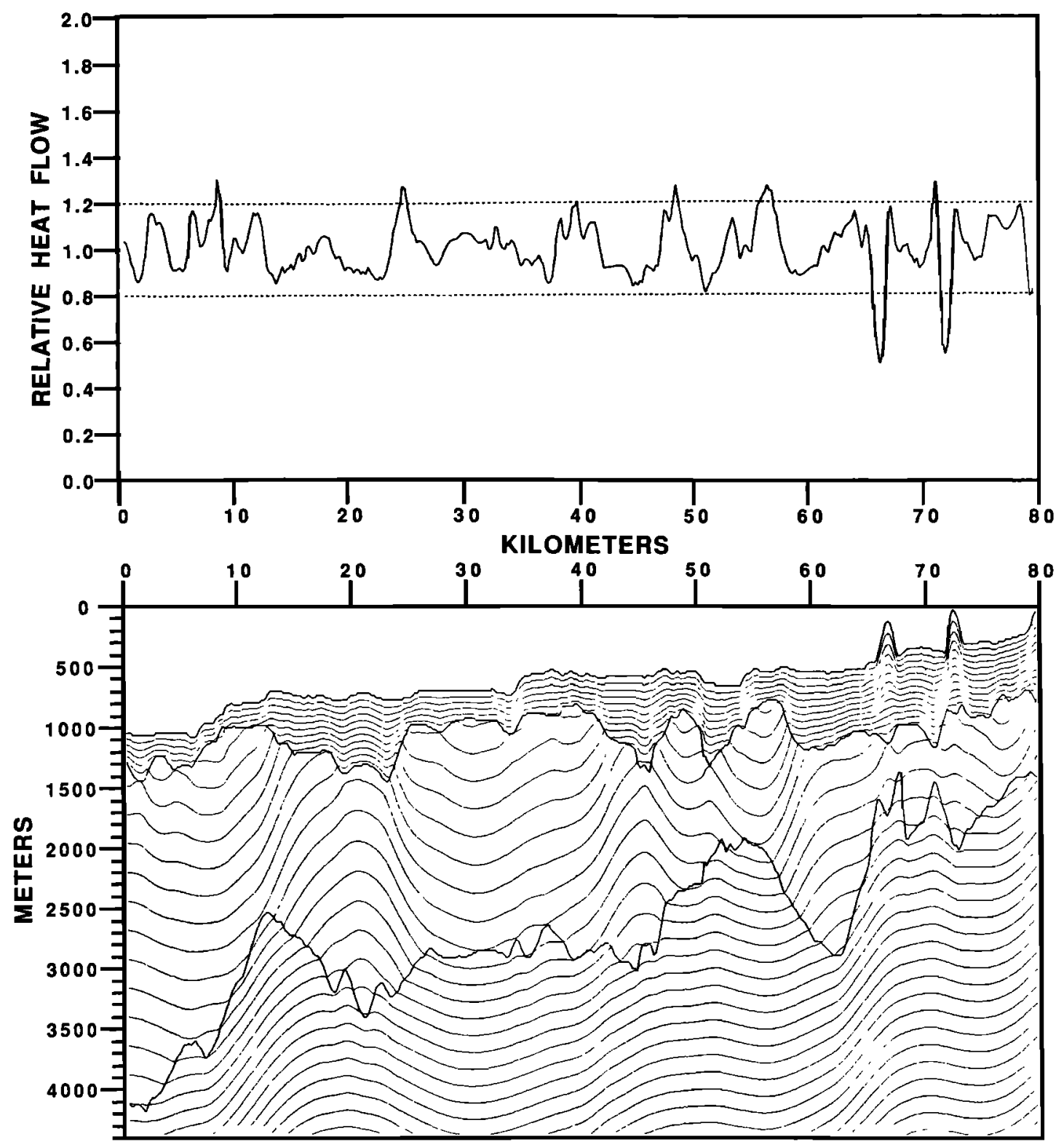

Fig. 5. Finite difference calculation of the effect of relief on sea bottom and evaporite layer on the relative vertical heat flow at the surface. Interfaces are digitized depth-converted reflectors from Exxon multichannel seismic line ERS74 following the interpretation of Barakat and Miller [1984]. This line is nearly coincident with the western part of the central heat flow line and its location is shown by the dash-dotted line on Figure 2. Top layer corresponds to postevaporite sediments with a thermal conductivity of $1.1 \mathrm{~W} \mathrm{~m}^{-1}{ }^{\circ} \mathrm{K}^{-1}$; middle layer to evaporites with a conductivity of $5.1 \mathrm{~W} \mathrm{~m}^{-1}{ }^{\circ} \mathrm{K}^{-1}$ and bottom layer to preevaporite sediments with a conductivity of $3.1 \mathrm{Wm}^{-10} \mathrm{~K}^{-1}$. Bottom boundary has a constant heat flow condition imposed. Isotherms shown with the layers are contoured at $10^{\circ} \mathrm{C}$ intervals. Note that heat flow variations are generally contained within $\pm 20 \%$ of the bottom flux.

material may be eolian and that the predominantly northerly winds in the northern Red Sea are responsible for their asymmetric distribution.

The number of thermal conductivity determinations and their distribution are inadequate in themselves to form a precise estimate of the uncertainty in the contour map of Figure 2. We thus adopt the maximum observed standard error within a core of $\pm 0.12 \mathrm{~W} \mathrm{~m}^{-1} \mathrm{~K}^{-1}$ as a bound on the uncertainty in thermal conductivity. We assign thermal conductivities to the measured thermal gradient values directly from the contour map of Figure 2 and assign uncertainties to the heat flow by the relation

$$
\Delta \mathrm{Q}=\mathrm{G} \Delta \mathrm{K}+\mathrm{K} \Delta \mathrm{G}
$$

where $\mathbf{Q}, \mathrm{G}$, and $\mathrm{K}$ are the heat flow, temperature gradient, and thermal conductivity, respectively, and $\Delta$ represents the uncertainty in the corresponding quantity .

\section{Sedimentation}

Sedimentation decreases measured heat flow from what it would be without sedimentation. The magnitude of the effect depends on the sedimentation rate, on the duration of the sedimentation, and on the thermal diffusivity of the sediments. 
The spatial and temporal variation of these parameters is poorly known for the Red Sea, and we attempt to estimate them based on the maximum plausible value that they might have. For the northern Red Sea, away from the coastal shelves at the location of the heat flow measurements, the disturbance caused by sedimentation is mainly due to the deposition of two distinct layers. The first is the post-evaporite mainly calcareous layer. The second is the evaporite layer which has been determined in the southern Red Sea to be composed primarily of halite and anhydrite with some interbedded sands and shales [Stoffers and Ross, 1974]. We neglect the effect of pre-evaporite sediments since they are older than about $15 \mathrm{Ma}$ and the sedimentation disturbance is strongly weighed toward the more recent sedimentation pattern. Figure 6 shows plots of the percent lowering of the surface heat flux for post-evaporite and evaporite layers. These results were calculated according to the analytical formulation presented by Langseth et al. [1980] assuming a constant sedimentation rate through time for individual curves and a constant heat flow condition at the base of the sediments.

In the northern Red Sea, post-evaporite sediment thicknesses are observed from nearshore wells to vary between about 150 and $600 \mathrm{~m}$ [Barakat and Miller, 1984; Miller and Barakat, 1988]. Seismic reflection profiles [Barakat and Miller, 1984; Martínez and Cochran, 1988; Miller and Barakat, 1988] indicate that the post-evaporite layer generally thins toward the center of the sea although thicknesses are quite variable. An

(a)
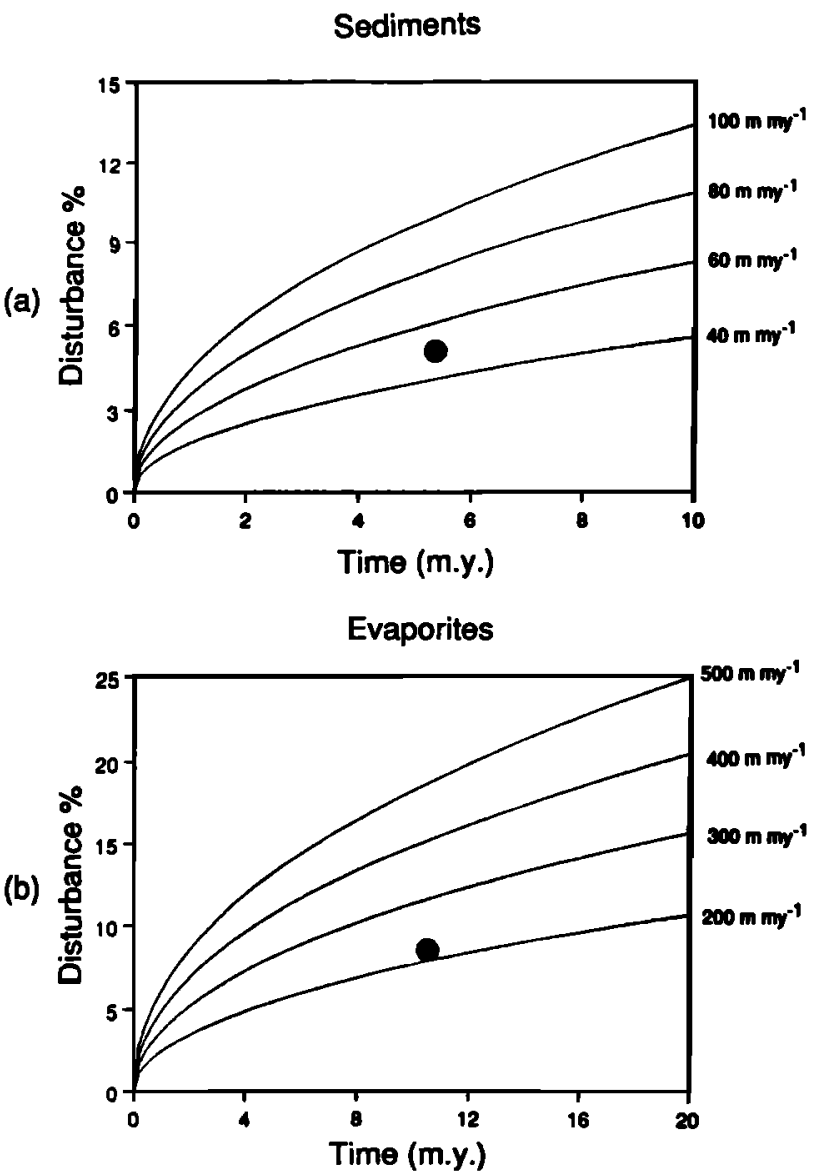

Fig. 6. Plots of the relative lowering of the vertical heat flux through time due to sedimentation at uniform rates. Calculation follows the analytical formulation of Langseth et al. [1980]. Solid circle shows value appropriate to the northern Red Sea. (a) Post-evaporite sediments with a thermal diffusivity of $2.0 \times 10^{-7} \mathrm{~m}^{2} \mathrm{~s}^{-1}$. (b) Evaporitic sediments with a thermal diffusivity of $2.6 \times 10^{-6} \mathrm{~m}^{2} \mathrm{~s}^{-1}$. average sedimentation rate of $50 \mathrm{~m} / \mathrm{m}$.y. for the post-evaporite sediments appears reasonable for the Recent sedimentation in the northern Red Sea [Herman and Rosenberg, 1969] and is compatible with the average thickness of the post-evaporite layer (approximately $250 \mathrm{~m}$ ). Using this rate, a heat flow disturbance of about $5 \%$ is calculated after $5 \mathrm{~m}$.y. of uniform sedimentation (see Figure 6a).

The thermal disturbance due to the evaporities is more difficult to assess due to the fact that its thickness variation is not well known and that it appears to have been deposited episodically over a period of about $10 \mathrm{~m}$.y. from middle to late Miocene time. The thickness of the evaporite layer determined from nearshore wells ranges from about 400 to $1200 \mathrm{~m}$ [Barakat and Miller, 1984; Miller and Barakat, 1988]. The evaporites are observed on seismic reflection profiles to thicken seaward. Based on seismic correlations from an extensive multichannel reflection survey in the western half of the northern Red Sea, Barakat and Miller [1984] and Miller and Barakat [1988] estimate evaporite thicknesses to locally exceed $2100 \mathrm{~m}$ toward the axis. Adopting an average thickness of $2000 \mathrm{~m}$ yields a mean sedimentation rate of 200 $\mathrm{m} / \mathrm{m} . \mathrm{y}$. This rate produces a disturbance of $7.6 \%$ at the end of evaporite deposition (see Figure 6b). The combined effect of the evaporite and post-evaporite deposition is less than the sum of these disturbances, however, since the thermal disturbance at the top of the evaporites will diminish in the intervening 5 m.y. of post-evaporite deposition.

The thermal disturbance due to sedimentation in the northern Red Sea may have rift-wide systematic trends since the evaporites tend to thicken toward the center of the rift while the post-evaporites thin. In addition, the heat flow at the base of the sediments has not remained constant, as assumed in the above calculation, but has increased in space and time across the rift. Given these uncertainties, the above calculation can only place a rough estimate, of the order of $10 \%$, on the sedimentation disturbance across the rift. An indication that the actual sedimentation disturbance does not significantly exceed this estimate is given by the observation that a continuation of the heat flow trend into Egypt matches very well with near coastal and land values presented by Morgan et al. [1985]. Morgan et al. [1985] concluded from the smooth continuation between land and marine values that neither erosion nor sedimentation were likely to be primary controlling agents on the heat flow. We thus neglect the relatively small sedimentation effect in light of the observed increase in heat flow of about $300 \%$ from the margins to the center of the rift.

\section{Hydrothermal Heat Transfer}

Indications of hydrothermal heat transfer come primarily from very large heat flow variations between closely spaced measurements and from slightly higher than normal bottom water temperatures. Systematic nonlinearities in individual temperature gradients are small and difficult to ascribe unambiguously to hydrothermal circulation because of the short length over which the gradient is measured and because of the uncertainty in the depth variation of the conductivities, which are observed to have systematic but variable trends with depth (see Figure 3).

Several locations where the heat flow variations tend to be anomalously large can be observed on the heat flow profiles shown in Figures 7a, 7b, and 7c. One of these locations occurs about $20 \mathrm{~km}$ to the east of the center of the northern heat flow transect (see Figure 7a). At this location, the heat flow profile crosses a structural boundary which has been interpreted from bathymetry and gravity data as a cross-trending fault zone referred to as an "accommodation zone" [Martínez and Cochran, 1988]. The accommodation zone forms the along-strike termination of sets of tilted fault blocks and takes up differential motion between the adjacent sets of fault blocks in what is probably a pervasively faulted area. The heat flow values within this $10-\mathrm{km}$-wide area (stations $6 \mathrm{q}-7 \mathrm{f}$ in Table 1a) 


\section{$a$ \\ Red Sea Heat Flow (Northern Line)}
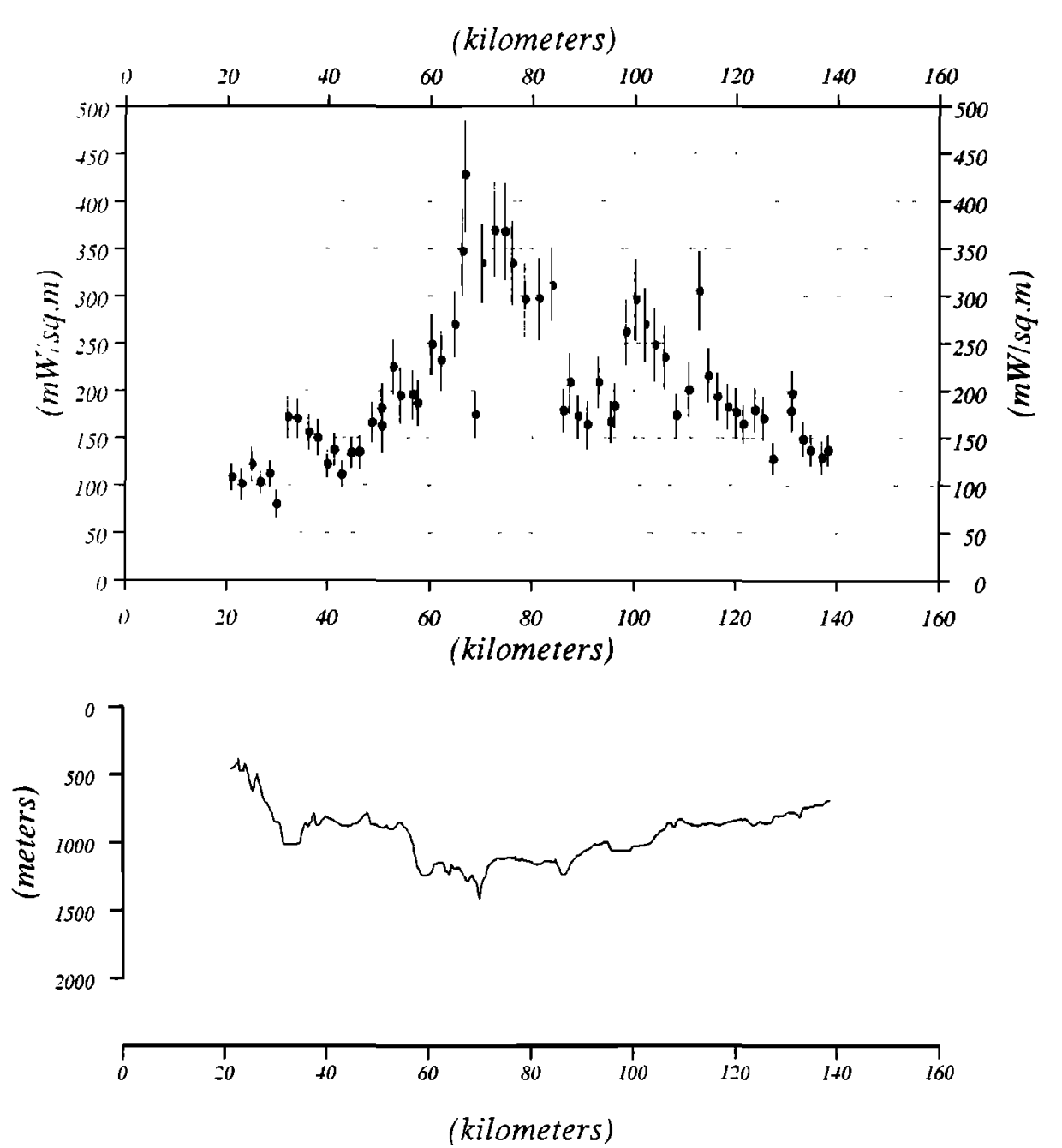

Fig. 7. Bathymetry in meters and heat flow values in $\mathrm{mW} \mathrm{m} \mathrm{m}^{-2}$ projected along an azimuth of $\mathrm{N} 60^{\circ} \mathrm{E}$, perpendicular to the trend of the rift. Error bars are based on the combined estimated uncertainty in therma conductivity and gradient measurement for each station (see text). (a) Northern heat flow transect. (b) Central heat flow transect. (c) Southern heat flow transect. (d) Data from all three transects stacked on the axial depression and fit with a degree 6 polynomial curve (solid curve) to form a smoothed estimate of the heat flow variation across the rift. Dashed curves indicate the probable range of variations due to thermal refraction effects (see Figure 5). Origin for horizontal scales is arbitrary.

are depressed by about $150 \mathrm{~mW} / \mathrm{m}^{2}$ from those immediately to the west and east. One of the temperature gradient measurements in the center of this depressed region (station 7c) exhibits a systematic upward concavity possibly indicating downward seawater advection.

At Conrad Deep, within the axial depression on the central heat flow line, an extreme variation in heat flow from 615 to $56 \mathrm{~mW} / \mathrm{m}^{2}$ occurs over a distance of about $1.5 \mathrm{~km}$. The high value occurs on the floor of the deep and the low value on its northern wall. The extreme variation observed here over a short distance and the presence of slightly warmer bottom water $\left(0.04^{\circ} \mathrm{C}\right.$ warmer in the deep) have been interpreted as indicating hydrothermal flow [Cochran et al., 1986].

Other individual measurements may be affected by hydrothermal flow, in particular those near faulted escarpments. However, anomalous bottom water temperatures or significant curvature in the temperature gradients were not observed in measurements other than those discussed. There is no indication of larger-scale systematic hydrothermal circulation.

\section{Heat Flow Pattern}

The large number of heat flow measurements and their distribution allow a good estimate to be made of the overall pattern of the heat flow variation across the rift and of its uncertainty. All three lines have the same general characteristics (see Figure 7). Values increase from about $125 \mathrm{~mW} / \mathrm{m}^{2}$ seaward of the coasts to peak values which occur consistently over the axial depression. The association of the peak heat flow values with the axial depression holds even for the northern line where the axial depression shifts toward the west in alignment with the entrance to the Gulf of Suez and is no longer in the center of the rift [Martinez and Cochran, 1988]. The heat flow high in the axial depression averages just above $250 \mathrm{~mW} / \mathrm{m}^{2}$ for the central and southern lines but is somewhat higher at about $300-350 \mathrm{~mW} / \mathrm{m}^{2}$ for the northern line. The heat flow trend can be extended landward toward Egypt and matches well with near-coastal values of about $100 \mathrm{~mW} / \mathrm{m}^{2}$ presented by Morgan et al. [1985]. The values of Morgan et 


\section{$b$ \\ Red Sea Heat Flow (Central Line)}
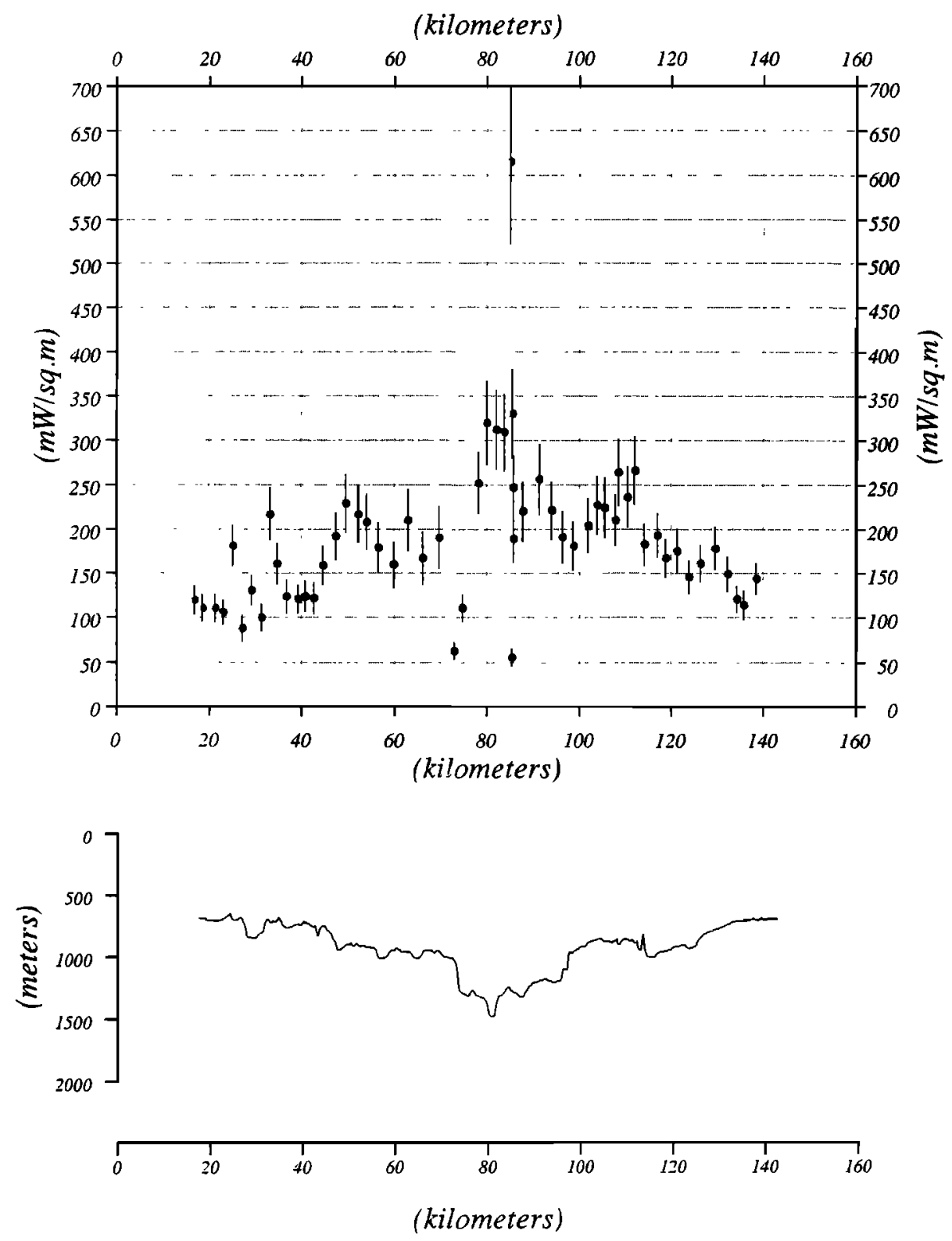

Fig. 7. (continued)

al. [1985] show that the thermal anomaly continues to decrease landward for about $30-40 \mathrm{~km}$ from the coast before attaining background values of about $35-55 \mathrm{~mW} / \mathrm{m}^{2}$ in the interior of Egypt. In Figure 7d the marine heat flow values presented here have been stacked on the axial depression and a degree 6 polynomial curve has been fit to the stacked data as a smooth estimate of the overall variation across the rift.

In summary, the scatter in the heat flow data can be attributed to a large degree to the refraction effect of the seafloor topography and relief on the upper surface of the evaporites. The scatter is distributed randomly and does not appear to have any systematic variation across the rift other than the predicted proportionality to the flux at depth and is generally contained within $20 \%$ of the average flux. Locally larger variations may be due to hydrothermal circulation, but do not affect the overall pattern. Systematic rift-wide effects are small. Sedimentation may account for an estimated $10 \%$ lowering of the heat flow across the rift. The thermal conductivities have systematic spatial variations which appear to be smoothly varying and have been simply contoured. The principal systematic variation due to conductivity should thus be accounted for. The remaining uncertainty in conductivity is assumed to be random and have a magnitude about equal to that of the individual cores. An estimate of the of the overall heat flow variation across the rift is obtained by the curve fit to the profiles stacked on the axial depression (Figure 7d).

\section{Numerical Models of an Extending Lithosphere}

\section{Model Description}

We employ a kinematic forward modeling technique to investigate the lithospheric thermal structure and surface heat flow generated by various simple geometries of extension and 


\section{$c$ \\ Red Sea Heat Flow (Southern Line)}
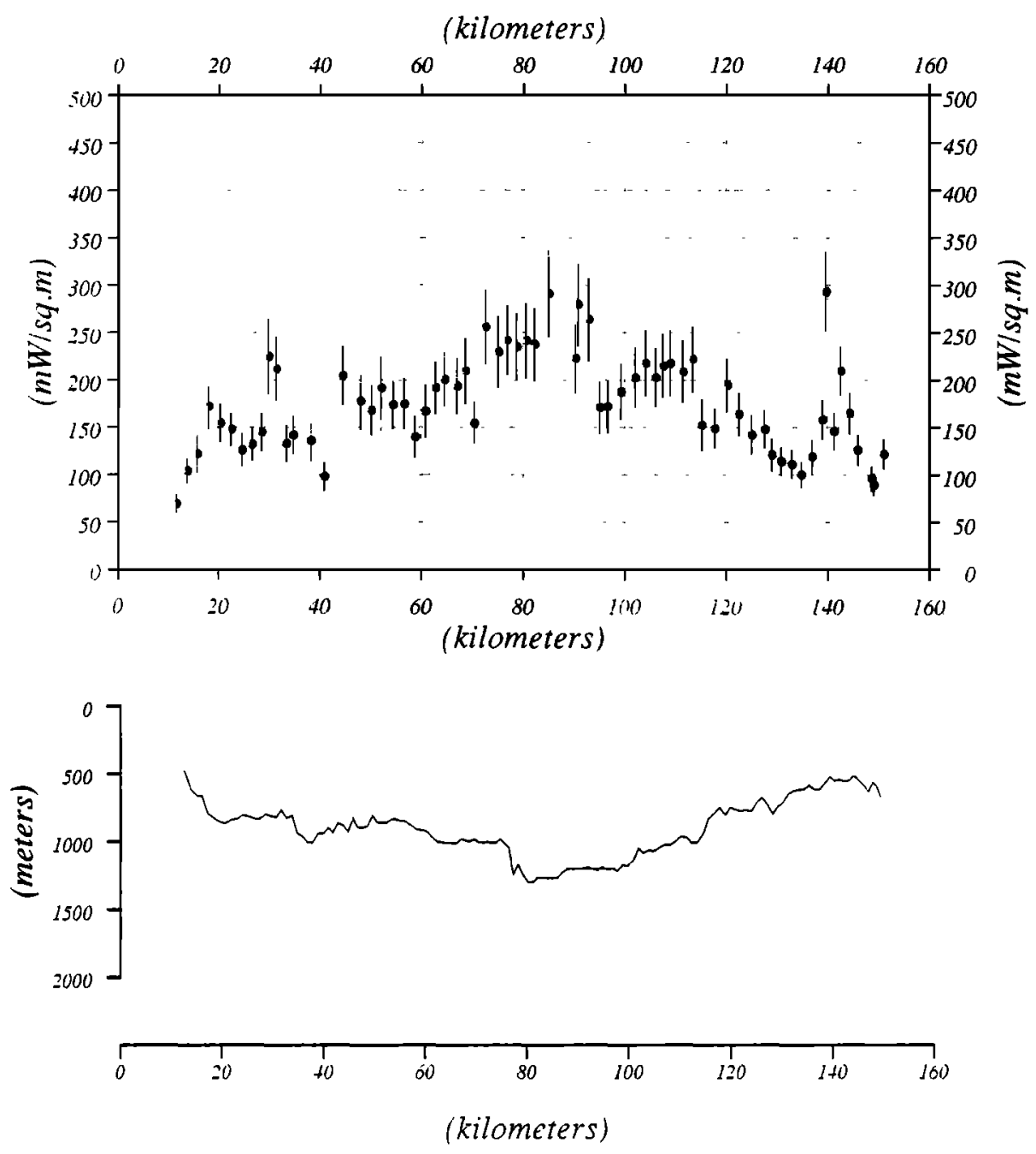

Fig. 7. (continued)

compare the results to observations from the northern Red Sea. The numerical technique uses a two-dimensional and time-dependent finite difference method described by Buck et al. [1988]. The model treats the advection of heat by prescribing the flow fields without regard to material strength or body forces. The method satisfies the continuity equation: $\partial \mathrm{u} / \partial \mathrm{x}=\partial \mathrm{w} / \partial \mathrm{z}$, however, which equates horizontal and vertical velocity gradients and thus conserves volume.

The rifting geometries considered are simple shear extension along a planar lithospheric detachment and pure shear extension within a zone that can change width in time. Heating results from lithospheric thinning and the asthenospheric flow required to conserve volume, but other sources of heat input such as induced convection are not considered. The model properties used are given in Table 4 . We neglect the relatively small crustal radiogenic heat contribution in these models. The model results are strongly dependent on the distribution of the extension within the rift and its development through time. We use the kinematic history of Red Sea opening to constrain the relative motion of the rigid plates landward of the border faults. Deformation seaward of the border faults is modeled as occurring in a variety of ways, as discussed below.

\section{Kinematic and Geologic Constraints}

The important plate kinematic parameters that determine the net lithospheric thermal anomaly are the total amount of opening and the rate of extension. The total amount of opening determines the total heat input into the lithosphere in the absence of convection. If volume is conserved, this heat can only be supplied from the vertical advection of material which fills in the space created by moving apart the lithospheric plates. The rate of extension determines how much time is available for heat loss through conduction and therefore how much of the original heat input from the asthenosphere remains for a particular geometry of extension.

The total amount of opening in the northern Red Sea can be determined from the amount and direction of opening in the Gulf of Suez and along the Aqaba-Levant shear zone. From geologic reconstructions of the tilted fault structure in the Gulf of Suez, Colletta et al. [1988] estimate a minimum of $20 \mathrm{~km}$ of total extension in the southern part of the Gulf. Steckler et al. [1988] arrive at a somewhat larger estimate of $32-36 \mathrm{~km}$ based on tectonic subsidence from a two-dimensional backstripping study. This larger amount is in good agreement with a recent 


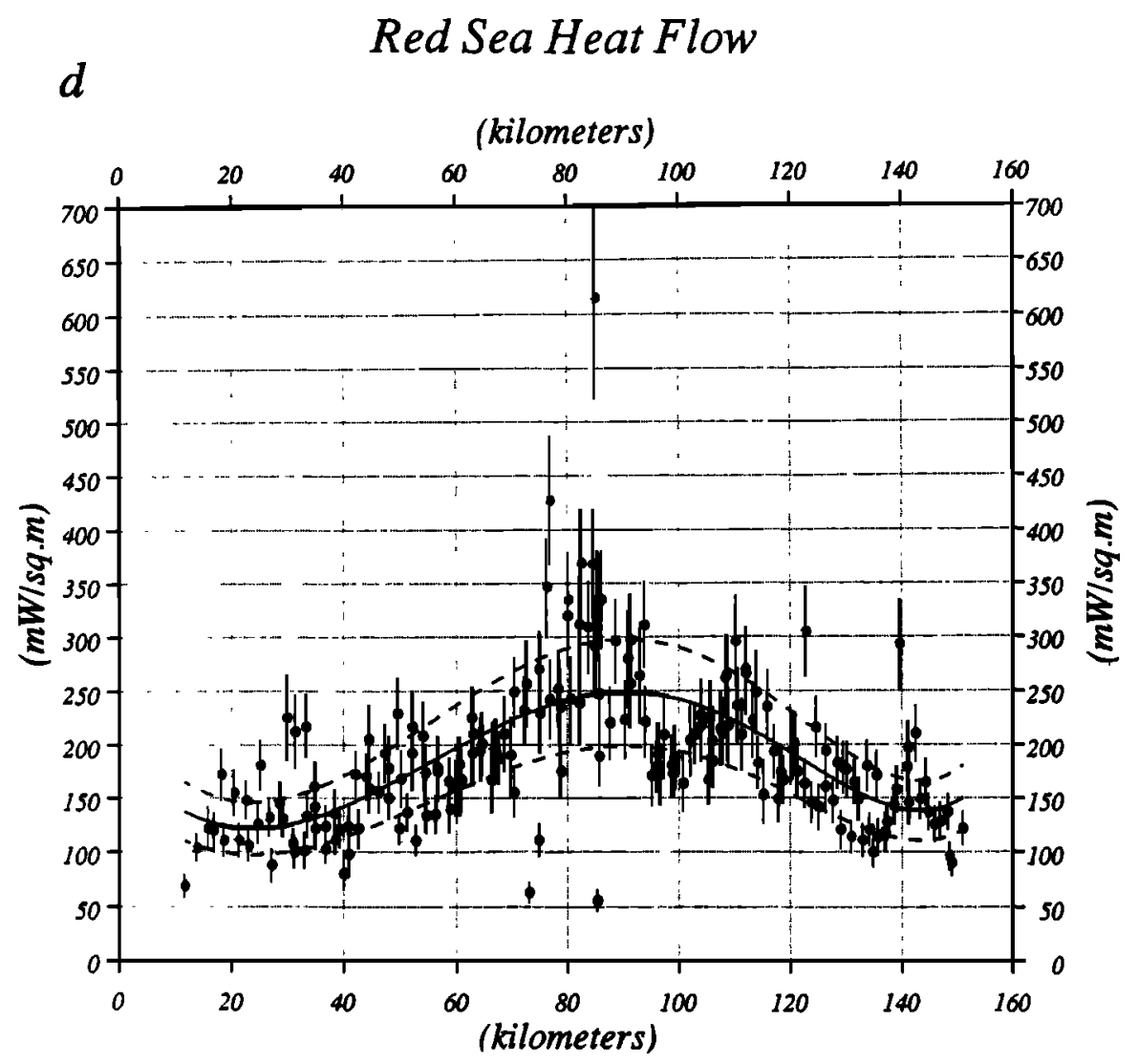

Fig. 7. (continued)

kinematic solution based on a simultaneous fitting of the plate motions for all of the plates in the region [Joffe and Garfunkel, 1987] which concludes that extension in the southern Gulf of Suez amounts to $35 \mathrm{~km}$.

Geologic mapping has determined that relative displacement along the Aqaba-Levant transform amounts to about $105 \mathrm{~km}$ of left-lateral shear [Freund et al., 1970; Garfunkel, 1981]. A1though the direction of opening through time for the Gulf of Suez is not well established and is certainly different from that along the Aqaba-Levant zone, adding these two displacements gives a maximum bound on the total Red Sea opening. The plate kinematic solution of Joffe and Garfunkel [1987] gives a total of $135 \mathrm{~km}$ of displacement at an average azimuth of $032^{\circ}$ at $27.5^{\circ} \mathrm{N}$ latitude in the Red Sea, which is in relatively good agreement with simply adding the two displacements described above. The opening direction is somewhat oblique to the trend of the northern Red Sea which is $335^{\circ}$. The opening perpendicular to the rift trend amounts to about $113 \mathrm{~km}$. Rates of opening are estimated by Joffe and Garfunkel [1987] for the

TABLE 4. Model Physical Constants

\begin{tabular}{lll}
\hline \multicolumn{1}{c}{ Parameter } & Value & Unit \\
\hline Crustal thickness & 32 & $\mathrm{~km}$ \\
Crustal density at $0^{\circ} \mathrm{C}$ & 2.8 & $\mathrm{~g} \mathrm{~cm}^{-3}$ \\
Lithospheric thickness & 125 & $\mathrm{~km}^{\circ}$ \\
Lithospheric density at $0^{\circ} \mathrm{C}$ & 3.33 & $\mathrm{~g} \mathrm{~cm}^{-3}$ \\
Thermal expansion coefficient & $3.4 \times 10^{-5}$ & ${ }^{\circ} \mathrm{C}^{-1}$ \\
Thermal conductivity & 3.2 & $\mathrm{~W} \mathrm{~m}^{-1}{ }^{\circ} \mathrm{C}^{-1}$ \\
Thermal diffusivity & $10^{-6}$ & $\mathrm{~m}^{-2} \mathrm{~s}^{-1}$ \\
Surface temperature & 0 & ${ }^{\circ} \mathrm{C}$ \\
Bottom boundary tempreature & 1300 & ${ }^{\circ} \mathrm{C}$ \\
\hline
\end{tabular}

northern Red Sea to amount to $0.75 \mathrm{~cm} \mathrm{yr}^{-1}$ total rate over the last $5 \mathrm{~m}$.y. Magnetic anomalies in the Gulf of Aden indicate that seafloor spreading has been taking place there at a nearly constant rate for 10-12 m.y. [Laughton et al., 1970; Cochran, 1981], and by inference that motion has probably also been taking place at a nearly constant rate in the Red Sea for at least that time allowing for some uncertainty introduced by the small amount of opening in the East African Rift.

In the models shown in Figure 8, we use maximum plausible values for the rate and amount of opening in the northern Red Sea rather than some "best" estimate because there still exists some controversy over the details of the kinematic history. Although the maximum values used in general cause an overestimation of the predicted heat flow and lithospheric heating, they serve to give an upper bound on the feasible heat input due solely to lithospheric extension. These models also illustrate the large differences resulting from the various geometries of extension even when they all have the same net heat input. In these models we use a constant total plate separation rate of $1 \mathrm{~cm} \mathrm{yr}^{-1}$ which takes up a total maximum of 140 $\mathrm{km}$ of northern Red Sea opening in $14 \mathrm{~m} . \mathrm{y}$. We also limit the extension to take place within a final rift width of $250 \mathrm{~km}$ in order to match the approximate present distance separating the border faults and flanking uplifted rift shoulder mountains of the northern Red Sea which form the limit of significantly thinned continental crust. These values are equivalent to a uniform overall present extension factor of $\sim 2.3$ within the rift. In Figure 9 we use different, more extreme, kinematic parameters proposed by LePichon and Gaulier (1988).

\section{Results}

Buck et al. [1988] model various forms of pure shear extension and simple shear extension occurring on a planar low angle detachment which cuts the entire lithosphere. We 
a
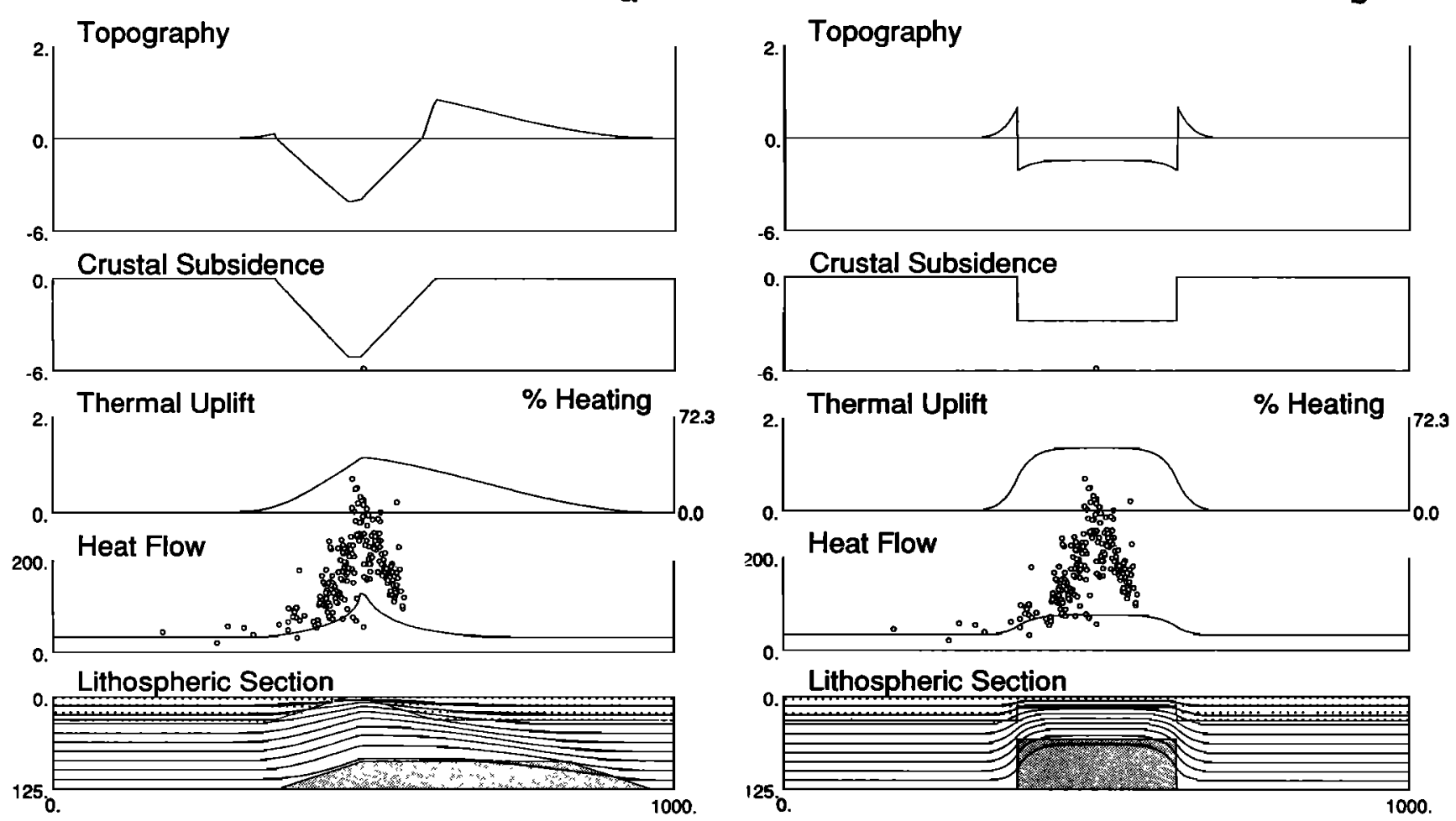

C
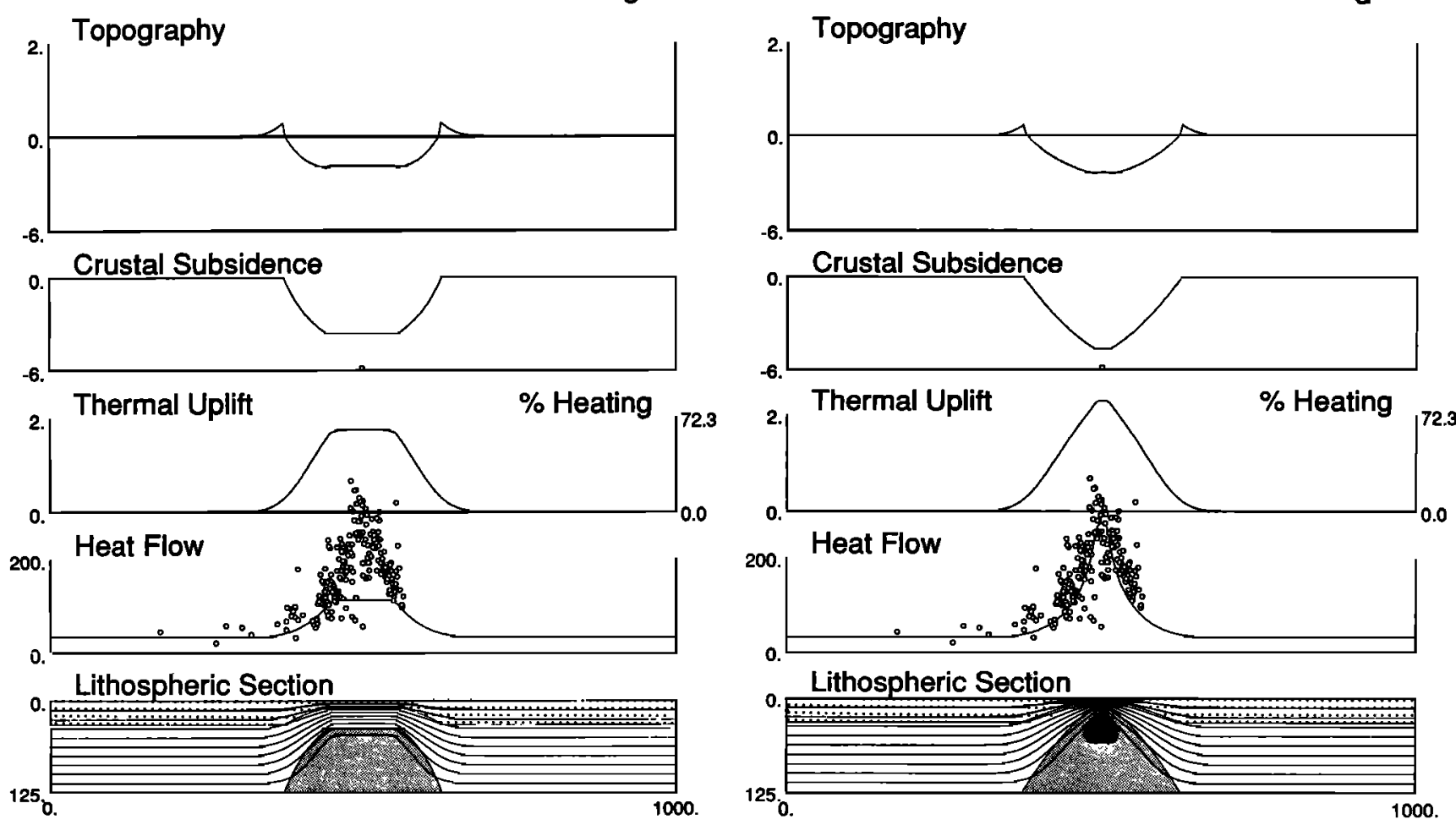

Fig. 8. Finite difference calculation, utilizing the technique of Buck et al. [1988], of the thermal structure of the northern Red Sea for (a) simple shear extension along a $15^{\circ}$ dip lithospheric detachment, (b) uniform pure shear extension within a widening zone, (c) pure shear extension within a constant width zone, (d) pure shear extension within a time-narrowing zone. Additional description is given in the text. 

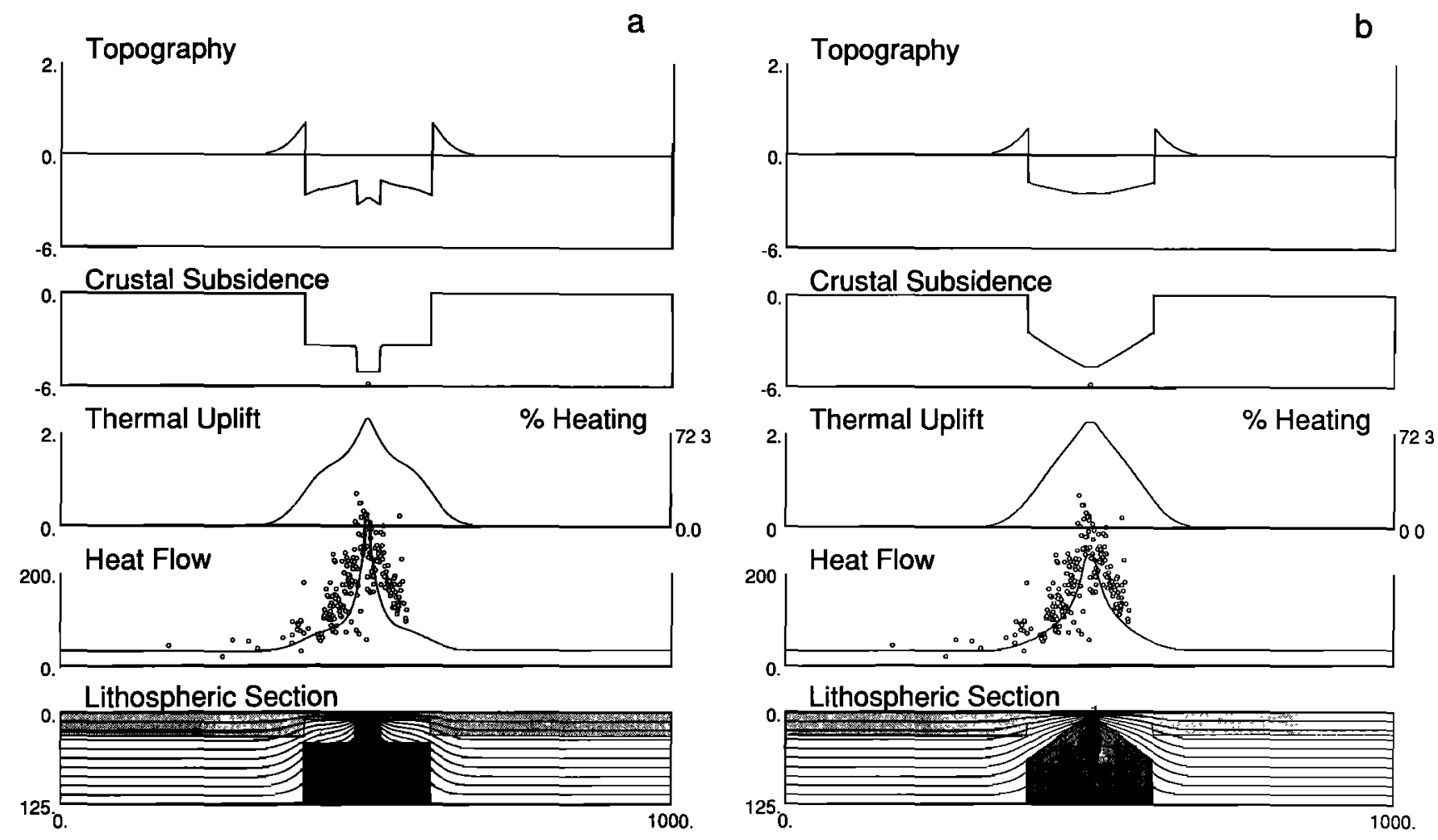

Fig. 9. Finite difference calculation for the thermal structure of the northern Red Sea for two different opening histories. (a) Rift extends uniformly until the crust is $10 \mathrm{~km}$ thick at which point the zone undergoing extension abruptly narrows to a width of $10 \mathrm{~km}$ simulating a seafloor spreading axis which is maintained for 5 m.y. (b) Rift extends uniformly until the crust is $16 \mathrm{~km}$ thick and then the zone undergoing extension gradually narrows to the width of the present axial depression, retaining $1.7 \mathrm{~km}$ of crust at the center. Both calculations use kinematic parameters from LePichon and Gaulier [1988]. Additional description is given in the text.

summarize the relevant principal results of that study below.

Results following the calculations of Buck et al. [1988] with slightly modified parameters are shown in Figures $8 \mathrm{a}, 8 \mathrm{~b}, 8 \mathrm{c}$, and $8 \mathrm{~d}$ for the simple shear case and three pure shear cases, respectively. In these figures, the bottom panel shows the lithospheric thermal structure. Isotherms are contoured at $130^{\circ} \mathrm{C}$ intervals with a temperature of $0^{\circ} \mathrm{C}$ at the top and $1300^{\circ} \mathrm{C}$ at the bottom of the lithosphere. The asthenosphere below $125 \mathrm{~km}$ depth is assumed to remain uniformly at $1300^{\circ} \mathrm{C}$. The crust is shown in stippling, and material originally advected from the asthenosphere is shown in grey.

The second panel from the bottom shows the calculated surface heat flow and the observed heat flow (shown as small circles) from this study and from Morgan et al. [1985] from boreholes and wells in Egypt.

The middle panel shows the component of local isostatic thermal uplift caused by the lithospheric heating. This can also be interpreted as the amount and distribution of lithospheric heating since it is calculated from the vertically integrated lithospheric thermal structure. If we consider the equilibrium geotherm in a vertical lithospheric column to represent $0 \%$ heating and a lithospheric column all at asthenospheric temperature (i.e., a column at a mid-ocean spreading center) to represent $100 \%$ heating, then the vertically integrated temperature greater than the equilibrium geotherm at any horizontal position is a measure of the "heating":

$$
\% \text { heating }=\left(\int\left(T(z)-T_{0}(z)\right) d z / \int T_{0}(z) d z\right) 100=H
$$

where $T_{0}(z)$ is the original equilibrium temperature variation with depth and $T(z)$ is the vertical temperature variation in a lithospheric column at a particular time after the start of rifting and the integration is over the original lithospheric thickness.

The second panel from the top shows the component of Airy isostatic subsidence caused by thinning of the crust. The top panel shows the locally compensated topography produced by adding the isostatic thermal uplift component to the crustal subsidence component.

In order to assess whether a model might be capable of generating melt we assume the following mantle solidus relation:

$$
\mathrm{T}_{\mathrm{o}}=1060^{\circ} \mathrm{C}+\left(4^{\circ} \mathrm{C} \mathrm{km}^{-1}\right) \mathrm{Z}_{\mathrm{o}}
$$

This relation describes melting that can first occur at a depth of $60 \mathrm{~km}$ if material having the original asthenospheric temperature is advected to this level. In equation (3), $T_{0}$ is the temperature at which melting first occurs, and $Z_{0}$ is the depth at which it first occurs. Regions exceeding this solidus relation are shown shaded in black if they occur in the lithospheric sections of Figures 8 and 9.

\section{Simple Shear Extension}

Rifting the lithosphere along a shallow dip detachment distributes the asthenospheric heat input in a very broad zone near the base of the lithosphere (see Figure 8a, bottom panel). The geometry of this model requires that the region of vertical advection beneath the detachment be as broad as the horizontal extent of the detachment. Relatively low horizontal temperature gradients are generated by this model. For the modeled conditions, advected material from the asthenosphere has risen to a depth of only $87 \mathrm{~km}$ and has appreciably cooled near the detachment. All points are well below the solidus (equation 
(3)). The heat flow maximum is centered in the rift, but is much narrower and has about half of the observed peak values (see Figure $8 \mathrm{a}$, second panel from bottom). The isostatic crustal component of subsidence is symmetrical (Figure 8a, middle panel), but because of the asymmetric heating of the lithosphere (Figure 8a, second panel from top), the hanging wall uplift is much larger than the footwall uplift (Figure 8a, top panel).

\section{Uniform Pure Shear Extension}

Figure 8b shows results of the calculation of pure shear extension within a region of uniform lithospheric thinning that widens with time. The initial width of the expanding region is $110 \mathrm{~km}$. Horizontal temperature gradients near the edges of the rift are larger than in the simple shear case and the asthenosphere has been advected to a higher level, in this case to a depth of $55 \mathrm{~km}$ (Figure $8 \mathrm{~b}$, bottom panel). However, according to the mantle solidus relation (equation (3)), no partial melting occurs. Peak calculated heat flow values (Figure $8 \mathrm{~b}$, second panel from bottom) in the center of the rift are only 75 $\mathrm{mW} / \mathrm{m}^{2}$, far below the observed mean peak values, and have a much broader and uniform distribution, very different from the markedly peaked observed heat flow distribution. Lithospheric heating and thermal uplift (Figure $8 \mathrm{~b}$, middle panel) are symmetrically distributed as is the resulting crustal subsidence (Figure $8 \mathrm{~b}$, second panel from the top) and topography (Figure 8b, top panel). Rift shoulder uplift values reach about $545 \mathrm{~m}$, significantly lower than those observed in Egypt of $\sim 800 \mathrm{~m}$ above base level.

\section{Constant Width Zone of Pure Shear Extension}

Figure 8c shows results of the calculation of pure shear extension within a constant width region which remains 110 $\mathrm{km}$ wide. The plate kinematic parameters are the same as before producing a rift having an overall extension factor of 2.3 but which varies exponentially from 1 at the edges of the rift to 3.6 within the $110 \mathrm{~km}$ wide thinning region and are uniformly 3.6 within this region. Higher maximum heat flow values of $113 \mathrm{~mW} / \mathrm{m}^{2}$ are produced in this model (Figure $8 \mathrm{c}$, second panel from bottom) but again are substantially below observed values and, in addition, do not have the form of the observed peaked heat flow pattern. The calculated rift thermal uplift and crustal subsidence (Figure 8c, middle panel and second panel from top) are symmetrical, as in the previous case, but maximum uplift of the rift shoulders (Figure $8 \mathrm{c}$, top panel) is significantly lower at $237 \mathrm{~m}$. Somewhat higher horizontal temperature gradients are produced in this model (Figure 8c, bottom panel). Asthenospheric material has risen to a depth of 35 $\mathrm{km}$ but has cooled by $260^{\circ} \mathrm{C}$ at the top to $1040^{\circ} \mathrm{C}$ which, according to the mantle solidus relation (equation (3)), will not generate melt.

\section{Time Narrowing Zone of Extension}

Figure $8 \mathrm{~d}$ shows the result of a calculation in which the zone of lithospheric extension becomes more narrow with time. This case assumes a constant rate for the narrowing of the zone of extension producing an extending region that corresponds roughly to the width of the current axial depression in the northern Red Sea, which appears to be taking up most of the present extension. Lithospheric thinning is totally confined within the zone of extension with material outside of the extending region moving horizontally only, without experiencing any additional relative deformation. The lithospheric thermal structure calculated for this model is shown in Figure 8d, bottom panel. The crust has a thickness which varies from $32 \mathrm{~km}$ at the edge of the rift to about $3 \mathrm{~km}$ in the central $20 \mathrm{~km}$ of the rift corresponding to a maximum extension factor of 11 . In this model, material crosses the solidus (equation (3)) and is shown in Figure 8d, bottom panel in black. The peak calculated heat flow value in the center of the rift is $274 \mathrm{~mW} / \mathrm{m}^{2}$. The heat flow pattern matches observed values in the center of the rift and the overall peaked trend although they generally fall slightly below observed values toward the coasts. Lithospheric heating (equation (2)) in the center of the rift (Figure $8 \mathrm{~d}$, middle panel) is $84 \%$ that of total heating to asthenospheric temperatures. Rift shoulder thermal uplifts (Figure 8d, top panel) are only $182 \mathrm{~m}$ for this model, far smaller than those observed in Egypt.

\section{Crustal Thickness Constraints on the Geometry of Extension}

A main conclusion of the study of Buck et al. [1988] was that the lithospheric extension had to become concentrated in a relatively narrow region in order to match the heat flow and to generate partial melt. The narrowing of the zone undergoing active deformation was modeled as a continuous process initiating at the start of rifting and occurring at a constant rate. This model is not unique with respect to matching the heat flow, however, and it is possible that the lithosphere may deform in other ways which are also compatible with the observations. Recently, new seismic refraction data from the northern Red Sea have become available that may help place additional constraints on the form of the lithospheric deformation. Gaulier et al. [1988] present two-ship seismic refraction data including expanding spread profiles (ESPs) which they interpret as indicating an abrupt thinning of the continental crust near the Egyptian coast. According to their interpretation, the crust thins near the coast from 35 to $20 \mathrm{~km}$. Seaward of the coasts, Moho depths become relatively uniform at about $14 \mathrm{~km}$ and the crust thins to less than $10 \mathrm{~km}$. These results have been interpreted by LePichon and Gaulier [1988] as indicating that the axial part of the northern Red Sea was created by seafloor spreading starting about $4.5 \mathrm{Ma}$. Their interpretation holds that lithospheric deformation in the northern Red Sea rift underwent an early period of relatively uniform extension followed by an abrupt concentration of extension and transition to seafloor spreading. We can model an extension history following the scheme of LePichon and Gaulier [1988] by allowing the region undergoing deformation to widen at the rifting velocity and then to narrow rapidly to a width of a few kilometers, simulating a seafloor spreading axis. Following a simplified plate kinematic interpretation from LePichon and Gaulier [1988], we model $150 \mathrm{~km}$ of extension which takes place within a final rift width of $200 \mathrm{~km}$ defined by the present separation of the coastlines. These parameters represent the maximum possible amount of extension and the minimum possible rift width which can be reconciled with the available geological and geophysical data. The zone of pure shear deformation starts out at about $50 \mathrm{~km}$ wide and is allowed to widen at a total rifting velocity of $0.75 \mathrm{~cm} \mathrm{yr}^{-1}$ until the crust is uniformly thinned from 32 to $10 \mathrm{~km}$. The deforming zone then abruptly narrows to a width of less than $10 \mathrm{~km}$, approximating a seafloor spreading axis, which is then maintained for $5 \mathrm{~m} . \mathrm{y}$. The results of this model are shown in Figure 9a. The calculated heat flow is generally compatible with observed values, although it is somewhat low in the marginal areas. A zone of melt is generated in the center of the rift and peak rift shoulder elevations are about $700 \mathrm{~m}$ for this model.

An alternative model which preserves a small amount of continental crust in the axis of the rift and is compatible with the crustal thickness variation reported by Gaulier et al.[1988] is shown in Figure 9b. In this model, the zone undergoing extension is allowed to widen at a total rate of $0.75 \mathrm{~cm} \mathrm{yr}^{-1}$ and to uniformly thin the continental crust to a thickness of 16 $\mathrm{km}$. The deforming zone then narrows to the width of the axial depression $(\sim 15 \mathrm{~km})$ by the time when the assumed total width of the present day rift is reached $(200 \mathrm{~km})$. This model retains a thickness of about $1.7 \mathrm{~km}$ of continental crust in the axial depression and also generally matches the peak heat flow values, although peak heat flow is somewhat lower than in the previous model. A region of melt is produced and rift shoulder elevations of about $570 \mathrm{~m}$ are created. 


\section{Discussion}

The numerical modeling described above is intended to place large-scale constraints on the kinematic geometry of lithospheric deformation in the northern Red Sea rift. The pattern of over $500 \%$ increase in heat flow across the rift from background values ranging from $35-55 \mathrm{~mW} / \mathrm{m}^{2}$ in the interior of Egypt [Morgan et al., 1985] to average peak values of over $250 \mathrm{~mW} / \mathrm{m}^{2}$ in the axial depression is difficult for most models to match even with the generous plate kinematic parameters used and provides a strong criterion for discrimination between the models. Another difference between the models is whether they predict generation of some degree of partial melt. This is a somewhat weaker measure for evaluating the models since it is dependent on the assumed solidus. It can, however, be used as a relative indicaton of whether a model is compatible with an ability to sustain some degree of melting. A third difference between the models is the predicted amount of rift shoulder uplift. The observed rift shoulder elevations are the combined result of thermal uplift and erosion and potentially also involve a flexural response. The models presented here only predict the thermal component, and even though we consider this to be the main contribution, they can only serve as rough approximations of the expected uplift. Despite this limitation, because the differences in predicted thermal uplift between the various models are substantial, the predicted uplifts are of value in qualitatively evaluating the relative ability of the models to satisfy the observations.

The central Red Sea is known to have seafloor spreading occurring in the large axial deeps, and active seafloor spreading is occurring in a continuous axial trough in the southern Red Sea. Some melt is known to exist in the northern Red Sea as it is presently reaching near the seafloor surface and generating the large magnetic intrusions observed. Most of these large intrusions occur in the axial depression in association with the deeps, but two large dipolar magnetic anomalies are also observed to occur in the marginal areas (see Figure 10). It is possible that there is melt underplating or intruding the lower crust in the marginal areas also. Unusual Moho seismic velocities $\left(7.2-7.5 \mathrm{~km} \mathrm{~s}^{-1}\right.$ ) which have been reported from expanding spread profile (ESP) refraction experiments in the northern Red Sea [Gaulier et al., 1988] may actually be due to intruded lower crust or to underplating from partial melt. Calculations carried out by Gaulier et al. [1988] indicate that high temperatures in the upper mantle are insufficient in themselves to explain the low velocities. Seismic experiments from the eastern margin of the U.S. [LASE, 1987] find similar velocities in the lower crust of this ancient margin where they cannot be attributed to abnormally high mantle temperatures. In old margins, the anomalous velocities are usually ascribed to either underplating or intrusion of the lower crust and not to anomalous mantle [Furlong and Fountain, 1986]. The anomalous velocities may thus imply that there has been or is more melting in the northern Red Sea than has reached near the surface as intrusions.

A large topographic asymmetry exists across the rift between the Egyptian and Arabian sides of the northern Red Sea. This asymmetry involves not only the magnitude of the rift shoulder elevation but also the base level inland away from the Red Sea. Average rift shoulder elevations are $1100 \mathrm{~m}$ on the Egyptian side and $1200 \mathrm{~m}$ on the Arabian side, while inland of the rift shoulder mountains, elevations decrease to about $300 \mathrm{~m}$ in Egypt but remain between 500 and $1000 \mathrm{~m}$ for at least $700 \mathrm{~km}$ on the Arabian side. As shown by the model calculation of Figure 8a, this extreme asymmetry cannot be primarily caused by the rifting alone. The cause of the asymmetry is treated to some degree by Buck et al. [1988], who advance two hypothetical explanations: The high regional uplift of the Arabian plate relative to the African plate across the Red Sea may be due to a long-wavelength flexure response to subduction and loading of the Arabian plate at the ZagrosTaurus collision zone. The asymmetry caused by this effect is significantly accentuated if the Arabian and African plates are decoupled across the Red Sea rift. The second cause for the asymmetry discussed by Buck et al. [1988] is that the motion of the Arabian plate relative to the deeper mantle could have caused the Afar hot spot to have passed under the uplifted areas of the Arabian plate. Regional uplift could thus have resulted if significant heating or underplating occurred. We consider both of these causes as potential explanations of the asymmetry, although neither a flexural upwarping of the Arabian plate nor the presence of an underplated layer have been established.

Simple shear extension along a shallow dip detachment has been proposed [Wernicke, 1985] to explain the observed asymmetric topography across the rift and the volcanism on the Arabian plate. The observed heat flow high cannot, however, be generated by this geometry of extension for the rates and total amount of plate separation of the northern Red Sea. Simple shear extension along a shallow dip detachment broadly distributes advection beneath the hanging wall plate which does not efficiently heat or thin the lithosphere. In the simple shear model, the crust becomes totally stripped off the center of the rift, but at no point is the advected lithosphere near the mantle solidus. With continued extension, the lithosphere eventually achieves a steady state temperature structure which is everywhere below the mantle solidus. This model therefore does not account for the heat flow pattern nor does it provide a mechanism for generating the melt needed for producing new oceanic crust and results in exposing mantle at the surface.

An aspect of the observed heat flow not totally explained by any of the models is the magnitude of the heat flow outside of the axial depression. Although the peak heat flow values in the center of the rift can be matched by the time-narrowing pure shear models, calculated values outside of the axial depression appear to be somewhat lower than the general pattern of observed heat flow values and may require an additional component of lithospheric heating. This is also suggested by the somewhat lower rift shoulder uplift generated by the models in Figure $8(<550 \mathrm{~m})$ which are significantly smaller than the observed rift shoulder elevation above base level in Egypt (800-1000 m) (Figure 11). The models in Figure 9 however, which assume a narrower rift and incorporate a period of uniform extension followed by a narrowing of the deforming region, generate higher rift shoulder uplift (> $570 \mathrm{~m}$ ).

Morgan et al. [1985] concluded that an additional source of heat was required in order to explain the nearshore thermal anomaly in the northern Red Sea. They show that it is unlikely that the nearshore thermal anomaly is due to erosion, an anomalous crustal radioactive component, or lateral heat flow from a spreading center without additionally emplacing intrusive melt into the crust near the coast. They propose that a prerifting active mantle heat source could supply this heat. However, Bohannon et al. [1989] present convincing evidence from the distribution of Oligocene marine sediments and soil horizons that the northern Red Sea was slightly below or near sea level prior to rifting and thus indicating that doming and active mantle heating did not precede rifting. The additional heating required could be supplied subsequent to the initiation of rifting by small-scale convection induced by the temperature structure established by the geometry of lithospheric rifting itself. Such a mechanism has been proposed to explain the rift shoulder uplift in the Gulf of Suez [Steckler, 1985; Buck, 1986 ] and the unusually voluminous early seafloor spreading magmatism of the Norway-Greenland conjugate margins [Mutter et al., 1988].

Alternative methods of redistributing lithospheric heating without induced convection have been proposed by Rowley and Sahagian [1986], Coward [1986], and White and McKenzie [1988]. In their models, the width of the region undergoing extension may vary with depth while maintaining the same integrated amount of extension in any horizontal 


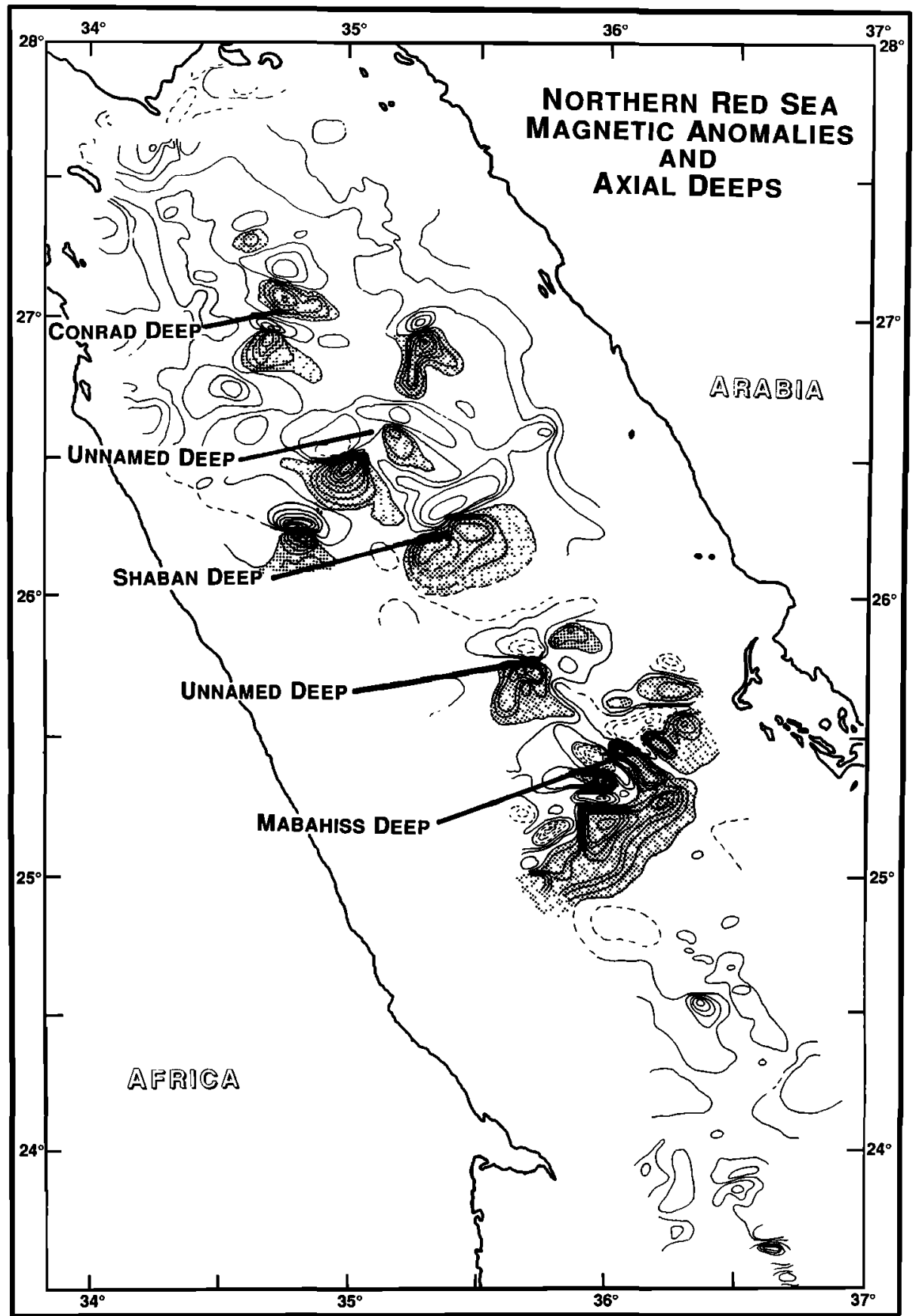

Fig. 10. Generalized magnetic anomaly map compiled from Martínez and Cochran [1988] and Guennoc et al. [1988]. Dipolar magnetic anomalies north of Mabahiss Deep are all normally magnetized and are generally associated with the regularly spaced deeps. Anomalies are contoured at 50-nT intervals. Dipolar anomalies north of $26^{\circ} \mathrm{N}$ are stippled if greater then $-200 \mathrm{nT}$ while dipolar anomalies south of $26^{\circ} \mathrm{N}$ are stippled if greater than $-100 \mathrm{nT}$.

plane. These models allow for subcrustal lithospheric heating beneath the unextended edges of the rift while satisfying volume conservation conditions which were not met in two-layer stretching models [e.g., Royden and Keen, 1980]. These models can be viewed as particular cases of the more general formulation of Dunbar and Sawyer [1988] in which vertical variations in the strength of the lithosphere determine the distribution of deformation. In the absence of convection, however, these models supply the same net heat input into the lithosphere as the modeling technique presented here since the net heat input is determined by the lithospheric thickness and the total amount of opening. Thus, although distributing lithospheric extension more broadly at depth beneath the unextended rift shoulder crust would increase the uplift, it would also decrease the surface heat flow. It therefore seems likely that even if lithospheric extension varies vertically in the northern Red Sea, an additional component of heating is still necessary in order to match heat flow in the marginal and near coastal areas.

If the lithosphere retains significant flexural strength during 


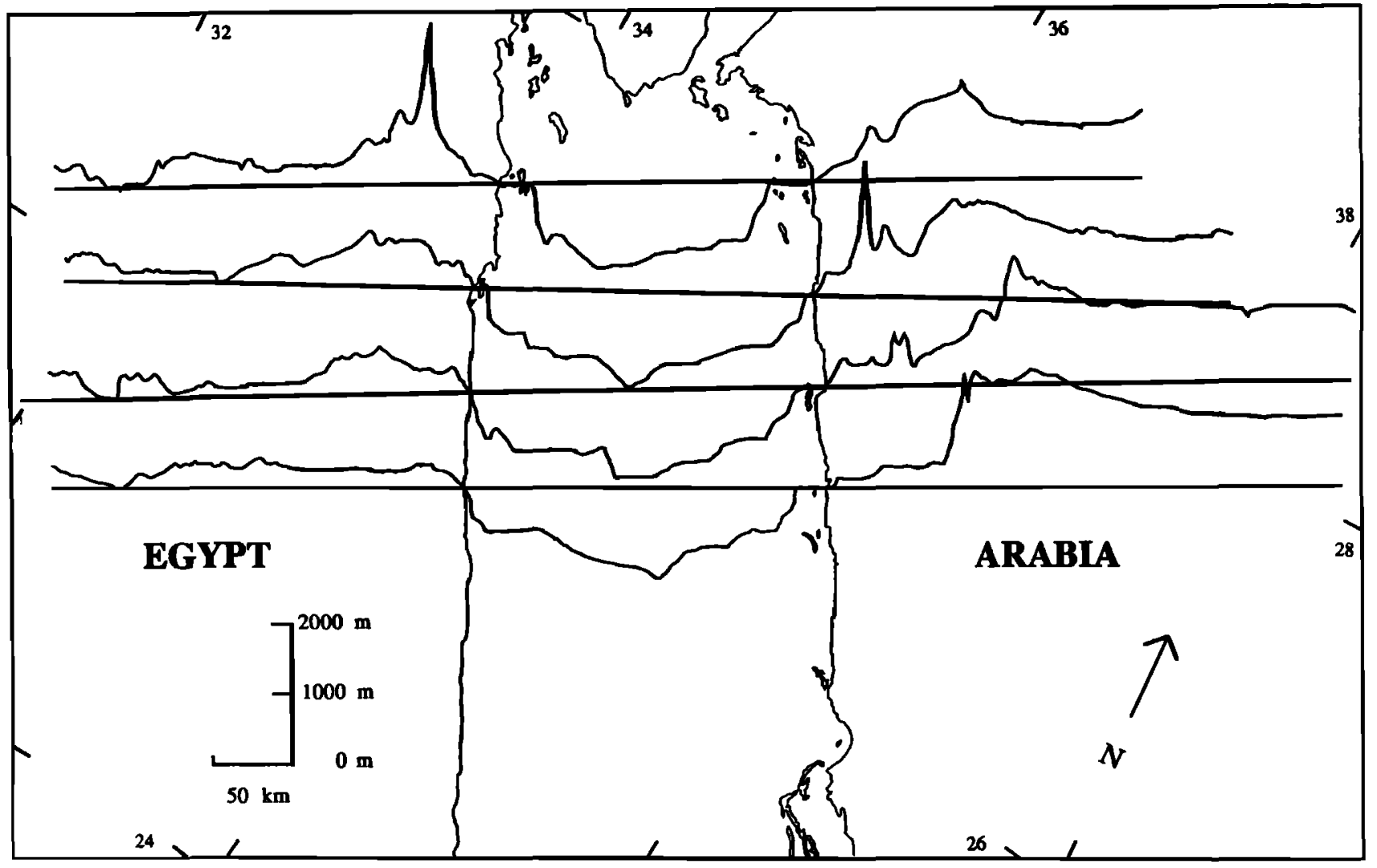

Fig. 11. Topographic profiles across the northern Red Sea. Elevations (or depths) are plotted perpendicular to baselines which serve to show location of the profiles. Note the asymmetry between Egypt and Arabia in baseline elevation away from the rift. Elevations above sea level are taken from 1:1,000,000 areonautical charts. Depths below sea level are taken from Martinez and Cochran [1988].

extension and if the extension results in the creation of buoyant forces which can be transmitted to the unextended rift shoulders then appreciable mechanical uplift may result. Whether this happens or not depends on the strength distribution of the lithosphere during extension and on the load distribution created by lithospheric deformation [Weissel and Karner, 1989]. The simple kinematic geometries assumed in our models do not permit us to quantitatively test these effects without additional constraints on the vertical distribution of deformation.

Our models indicate that extension cannot now be taking place uniformly across the entire width of the rift. The observed heat flow pattern is not matched by uniformly thinning the lithosphere within the rift (Figure 8b) nor by maintaining the zone of extension at a constant width (Figure 8c). Rifting in the northern Red Sea requires a concentration of extension in order to match the average peak heat flow values ( $>250$ $\mathrm{mW} / \mathrm{m}^{2}$ ). The extending region must, however, have been at some point wider than this in order to match the observed present width of the rift.

The heat flow pattern by itself is consistent with several scenarios in which the zone of extension eventually becomes narrow, including models which both do and do not involve a period of seafloor spreading (Figure. 9). Additional information is needed to further constrain the distribution of extension through time. New geophysical data which bear on the form of extension in the northern Red Sea have recently become available. These data include (1) two-ship seismic refraction data [Gaulier et al., 1988] which imply a thin crust, but indicate that the basement has continental seismic velocities within the heat flow survey area, (2) gravity data [Martínez and Cochran, 1988] which suggest a structural pattern consisting of tilted fault blocks within the marginal areas of the northern Red Sea, (3) Seismic reflection profiles [Martinez and Cochran, 1988] (see also Figure 12) which indicate that intense deformation is presently confined primarily to the axial depression; the profiles suggest that tectonic activity was formerly more widely distributed, and (4) magnetic anomalies which provide evidence for only localized intrusions; the existence of a continuous seafloor spreading axis cannot be demonstrated from the magnetic data.

An important factor in resolving the current form of lithospheric extension is to determine the structure and tectonics of the axial depression. The axial depression appears to form a distinct tectonic province. Gaulier et al. [1988] and LePichon and Gaulier [1988] identify three tectonic areas in the northern Red Sea mainly on the basis of seismic velocities. Gaulier et al. [1988] found that their northern set of profiles, near $27^{\circ} \mathrm{N}$ in the region of our heat flow profiles, showed continentaltype basement velocities beneath the marginal areas. Their southern set of profiles, south of the Brothers Islands at $26^{\circ} 10^{\prime} \mathrm{N}$, gave higher velocities under the marginal areas which they interpret as oceanic crustal velocities. Cochran and Martinez [1988], on the other hand, interpret these velocities as reflecting a change to a distinctly more mafic continental crust observed on shore [Stern et al., 1984]. Seismic velocities reported by Gaulier et al. [1988] for the axial depression are anomalously low on both the northern and southern portions of their survey. They interpret the refraction results from the axial depression as defining a third distinct region: "The third zone is the axial one... it has a complex structure with somewhat lower crustal velocities which might be due to a highly fractured and newly intruded crust..." [Gaulier et al., 1988 , p. 71].

LePichon and Gaulier [1988] interpret the axial depression 
SW

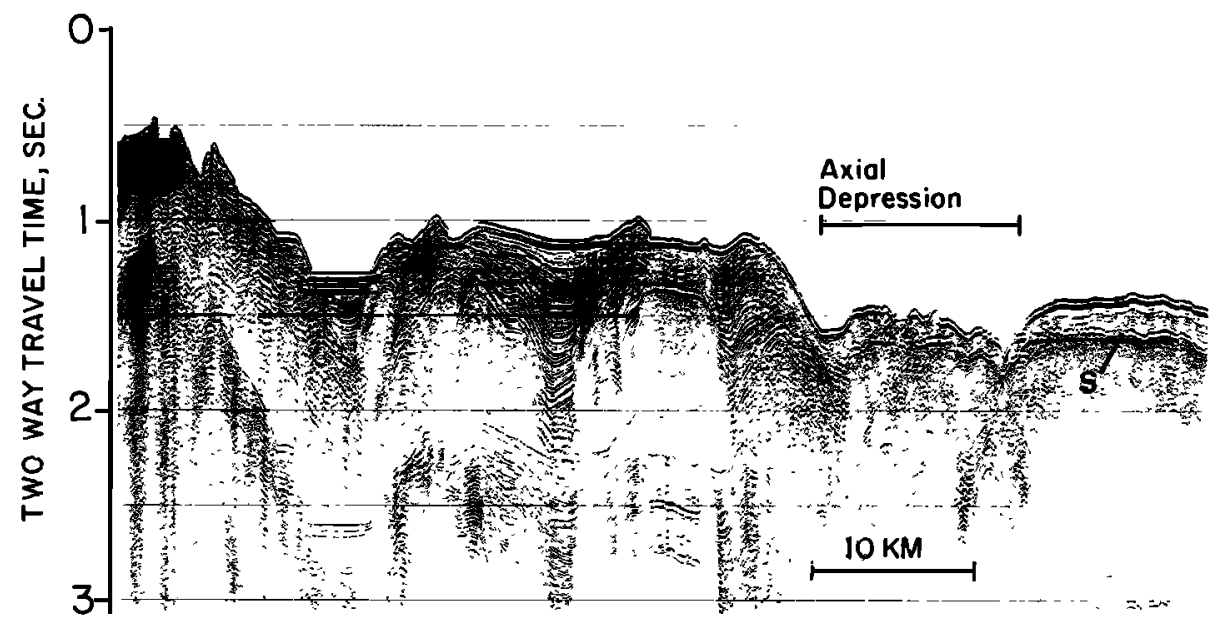

NE

Fig. 12. Water gun seismic reflection profile from the western half of the rift demonstrating the difference in the style and intensity of disturbance to the sediments between the marginal areas and the axial depression. Reflector labelled "S" represents the top of the evaporites. Profile roughly corresponds to the western half of the northern heat flow line and its location is shown by the dashed line in Figure 2.

as a seafloor spreading axis that has been accreting oceanic crust for $4.5 \mathrm{~m} . \mathrm{y}$. in the north and for perhaps $11 \mathrm{~m}$.y. in the south. They argue that seafloor spreading has initiated along the length of the axial depression but that it is obscured by the overlying sediments and halokinetic flow of the evaporites. However, thermal calculations based on the heat flow indicate that for reasonable conductivities, the sediments and upper few kilometers of crust should be below the magnetic blocking temperature [Cochran and Martínez, 1988] and that intruded material should acquire a magnetization. It has been suggested that the overlying sediments might cause intruded material to be poorly magnetized as a result of intense hydrothermal alteration [Levy and Riddihough, 1986] or very slow cooling [Girdler, 1985]. However, highly magnetic intrusions overlain by significant sedimentary cover, including evaporites, occur in conjunction with some of the regularly spaced northern Red Sea axial deeps (Figure 10). For example, highly magnetic intrusions occur in the vicinity of the unnamed deep at $25.8^{\circ} \mathrm{N}$ which is totally covered with sediments, including the evaporites [see Gaulier et al., 1988, Figure 14] and at Conrad Deep which is also completely sediment covered and in addition displays evidence of hydrothermal circulation [Cochran et al., 1986]. Thus it appears that where intruded magma is able to come near the surface in the northern Red Sea, it will cool below the blocking temperature and acquire an intense magnetization which contrasts strongly with that of the surrounding material.

These geophysical data are thus most compatible with a model for the northern Red Sea in which the marginal areas were formed by a period of diffuse continental extension which was followed by concentration of the extension into a narrow width region forming the axial depression. Although the axial depression is now taking up most of the extension, it appears not to be presently undergoing seafloor spreading but rather is undergoing the initial stages in the nucleation of spreading cells [Martinez and Cochran, 1988; Cochran and Martínez, 1988]. The only seismic velocity from the axial depression characteristic of igneous crust was obtained on ESP 14 of Gaulier et al. [1988] which passes directly over Conrad Deep, suggesting that large-scale intrusion is limited to specific locations characterized by large dipolar magnetic anomalies (Figure 10).

The northern Red Sea deeps have a similar spacing to the larger deeps in the central Red Sea where clear isolated centers of seafloor spreading occur [Bonatti, 1985]. This leads us to propose that northern Red Sea deeps represent an earlier stage in the establishment of organized seafloor-spreading centers. These centers are just now nucleating within axial depression where the crust has been locally breached. Additional support for local crustal rupture at the deeps comes from the seismic results reported by Gaulier et al. [1988]. Their ESP 14 crosses Conrad Deep, and their constant offset wide aperture reflection line R4 crosses the unnamed deep between Shaban and Conrad deeps. At these locations, the top of the crust abruptly deepens (line R4 over the unnamed deep) or the bottom of the crust is unusually shallow (ESP 14 crossing Conrad Deep). These observations suggest an abrupt local thinning or rupturing of the crust in the vicinity of the deeps.

Although the overall heat flow pattern in the northern Red Sea indicates that the lithospheric thermal structure must be similar to that beneath a seafloor spreading center, the actual initiation of seafloor spreading appears to be controlled by crustal structures developed during continental rifting. Periodically spaced, cross-trending accommodation zones have been identified in the northern Red Sea, primarily on the basis of discontinuities in the pattern of gravity highs and lows [Martinez and Cochran, 1988]. The three northernmost axial deeps, located within our detailed survey area, appear to be located halfway between the accommodation zones. We suggest that the accommodation zones are relatively broad, highly fractured zones in which extension is taken up somewhat diffusely across the rift. Between these bands, the crust breaks in larger and more coherent blocks which, near the center of the rift, can form localized and more concentrated centers of extension.

Evidence of periodic accommodation zones from the East African rifts [Bosworth, 1985; 1987; Bosworth et al., 1986; Ebinger et al., 1984] and from the Gulf of Suez [Moustafa, 1976; Steckler et al., 1988; Colletta et al., 1988] suggests that this structural periodicity may be established at an early stage in the development of a rift and may persist through to the initiation of seafloor spreading. The regular spacing which is evident in the location of the axial deeps north from Mabahiss Deep (Figure 10) is present in the central Red Sea deeps and is also present in the pattern of magnetic and tectonic lineation discontinuities within the active seafloor spreading axis of the southern Red Sea [Bonatti, 1985].

Bonatti [1985] first noted the periodicity of the central Red Sea deeps and its correlation with regularly spaced tectonic and magnetic lineation discontinuities which can be seen in GLO- 
RIA sidescan sonar data [Garfunkel et al., 1987] from the continuous spreading axis of the southern Red Sea. He attributed this pattern to periodic nucleation of seafloor spreading controlled by asthenospheric upwellings and proposed a "punctiform" model for the initiation of seafloor spreading at "hot points." The heat flow values in Conrad deep are locally very high and quite variable as would be expected for a localized intrusion. However, the average heat flow variation in the axial depression does not appear to correlate with the location of deeps, as might be expected if the deeps were a consequence of larger asthenospheric upwellings. When heat flow values in the axial depression are averaged for each of the three traverses we find that values for the central line crossing Conrad Deep are about the same as for the southern line which does not cross a deep and are somewhat higher for the northern line which also does not cross a deep. Heat flow compilations from the axial region of the central and southern Red Sea [Haenel, 1972; Girdler and Evans, 1977] also do not show periodic variations. In addition, it has been suggested that periodic asthenospheric upwellings should correspond to ridge axis highs [Crane, 1985]; in contrast, the periodic intrusions of the northern Red Sea and the seafloor spreading cells of the central Red Sea occur within wider bathymetric lows centered around the deeps and not at domal highs.

We propose that the sites of initiation of seafloor spreading are controlled by the distribution of crustal extension and the location of its final rupturing. This appears to be determined by two factors in the northern Red Sea. The first factor is the overall increase in the amount of extension and crustal thinning from the margins toward the center of the rift. The second factor is the local focusing of crustal extension at the deeps within the axial depression. This focusing appears to be controlled by the location of the regularly spaced cross-trending fault zones along the rift which may act to concentrate the extension near the center of the block faulted areas while distributing it more broadly within the cross-trending fault zones. Evidence from the Gulf of Suez and East African rifts indicates that this structural pattern is developed early in the evolution of a rift and that the periodicity is therefore not a consequence of the evolving deeper thermal anomaly since it precedes it.

\section{Conclusions}

Three heat flow profiles across the water-covered portion of the northern Red Sea rift show a systematic increase from values of about $125 \mathrm{~mW} / \mathrm{m}^{2}$ seaward of the coasts to average peak values of greater than $250 \mathrm{~mW} / \mathrm{m}^{2}$ associated with the axial depression. The heat flow pattern across the northern Red Sea rift exhibits a 500\% increase from background values of between $35-55 \mathrm{~mW} / \mathrm{m}^{2}$ in the stable interior of Egypt. Environmental effects were evaluated and shown not to significantly change the overall heat flow pattern. The largest estimated systematic disturbance results from sedimentation which could serve to lower the heat flow by an amount of the order of $10 \%$.

Two-dimensional time-dependent numerical models of various simple geometries of extension across the rift predict very different patterns of lithospheric heating, surface heat flow, rift topography, and potential for melt generation in the northern Red Sea. The large variation in heat flow across the rift, along with maximum bounds on the total amount and rate of plate separation, place strong constraints on the form of lithospheric extension within the rift.

The modeled simple shear extension across a shallow dip planar detachment does not match the observed heat flow and does not lead to thermal anomalies high enough to approach the mantle solidus. Horizontal temperature gradients generated by this rifting geometry are also low and unlikely to induce additional heating by a mechanism such as small-scale convection.

Uniform pure shear extension in a constant width or widening zone of extension also significantly underestimates the ob- served heat flow values and does not generate conditions approaching the mantle solidus anywhere in the lithosphere. Horizontal temperature gradients are somewhat higher near the edges of the rift for this model, and the possibility exists that small-scale convection might generate additional heating.

A simple rifting model in which a zone of pure shear extension narrows with time can match the overall pattern of heat flow across the rift and peak values in the center of the rift, satisfies limiting plate kinematic constraints, and also predicts the generation of some degree of melt within the rift. Heat flow values in the marginal areas are still lower than observed, as are rift shoulder elevations. High temperature gradients generated by this model, however, are consistent with the possibility for inducing small-scale convection which would augment the heat flow and thermal uplift. Additional geophysical data, however, suggest a modification of this model.

Seismic refraction data on the distribution of crustal thickness mainly from the western side of the rift reported by Gaulier et al. [1988] provide additional constraints on the rifting geometry. It appears from these data that extension may have been more uniformly distributed across the rift for some period of time, before becoming concentrated within the axial depression. This is consistent with sedimentary and structural relationships observed on seismic reflection records which indicate that tectonic activity has decreased with time in the marginal areas, while intense deformation remains active in the axial depression [Cochran et al., 1986; Martínez and Cochran, 1988].

LePichon and Gaulier [1988] suggest that extension became concentrated to the extent that a seafloor spreading axis formed approximately $4.5 \mathrm{Ma}$. However, crustal velocities in the axial depression [Gaulier et al., 1988] are in general not typical of igneous intrusive rocks except for one line near Conrad Deep. The occurrence of regularly spaced deeps associated with normally polarized dipolar magnetic anomalies suggests that melt has only recently started to reach near the surface as intrusions at these specific locations. Our model study shows that an initial period of uniform extension, followed by narrowing of the region of extension can satisfy both the geothermal and crustal thickness constraints and maintain a finite thickness continental crust at the axis.

The present-day axial depression represents the locus of maximum lithospheric heating and thinning and is very close to establishing an oceanic spreading center. The emplacement of periodically spaced intrusions at the axial deeps may mark the initial stages in the development of seafloor spreading cells. The regular spacing of the intrusion-associated deeps appears to be controlled by the pattern of crustal breakup rather than asthenospheric upwellings. The deeps occur halfway between cross-trending fault zones that form along-strike terminations to the tilted fault blocks that underlie the marginal areas of the northern Red Sea. The cross-trending fault zones take up the extension more diffusely than the more coherent fault blocks resulting in more concentrated extension forming the deeps. If these deeps continue to serve as loci for further intrusions, they may eventually develop into localized seafloor spreading cells as now occur in the central Red Sea.

Acknowledgments. We would like to express our gratitude to the governments of Egypt and Saudi Arabia for granting clearance to work in their waters. We would like to thank Captain J. Peterlin, Science Officer J. Smith and all of the officers and crew of the R/V Robert D. Conrad for their enthusiastic help. The shipboard conductivity measurements were carried out by M.A. Hobart. G.A. Dehgheni, M.S. Steckler, and U.S. ten Brink participated in the field program and aided greatly in collection of the data. We would like to thank Esso Exploration and in particular P. Miller and H. Barakat for their interest and for providing a copy of seismic line ERS74. Various drafts of this paper were reviewed by D. Sawyer, K. Klitgord, M. Langseth, W. Menke, W. Ryan, M. Steckler, C. Stein, A. Watts, and J. Weissel. We would particularly like to 
acknowledge extensive valuable discussions with $\mathrm{M}$. Langseth and M. Steckler. P. Wessel kindly provided computer graphics software. This work was supported by National Science Foundation grant OCE 83-09983 and OCE 87-16701. Lamont-Doherty Geological Observatory contribution number 4476.

\section{References}

Bäcker, H., K. Lange, and H. Richter, Morphology of the Red Sea central graben between Subair Islands and Abul Kizaan, Geol. Jahrb., D13, 79-123, 1975.

Barakat, H., and P.M. Miller, Geology and petroleum exploration, Safaga Concession, Northern Red Sea, Egypt, Seventh Exploration Seminar,Egypt. Gen. Pet. Corp. 26 pp., Cairo, 1984.

Berggren, W.A., Micropaleontologic investigations of Red Sea cores - Summation and synthesis of results, in Hot Brines and Recent Heavy Metal Deposits in the Red Sea, edited by E.T. Degens, and D.A. Ross, pp. 329-335, Springer-Verlag, New York, 1969.

Berggren, W.A., and A. Boersma, Late Pleistocene and Holocene planktonic foraminifera from the Red Sea, in Hot Brines and Recent Heavy Metal Deposits in the Red Sea, edited by E.T. Degens, and D.A. Ross, pp. 282-298, Springer-Verlag, New York, 1969.

Birch, F., The effects of Pleistocene climatic variations upon geothermal gradients, Am. J. Sci., 246, 729-760, 1948.

Bohannon, R.G., C.W. Naeser, D.L. Schmidt, and R.A. Zimmerman, The timing of volcanism and rifting periphera to the Red Sea: A case for passive rifting?, I. Geophys. Res., 94, 1683-1702, 1989.

Bonatti, E., Punctiform initiation of seafloor spreading in the Red Sea during transition from a continental to an oceanic rift, Nature, 316, 33-37, 1985.

Bonatti, E., P. Colantoni, B. Della Vedova, and M. Taviani, Geology of the Red Sea transitional region $\left[22^{\circ} \mathrm{N}-25^{\circ} \mathrm{N}\right]$, Oceanol. Acta, 7, 385-398, 1984.

Bosworth, W., Geometry of propagating continental rifts, Nature, $316,625-627,1985$.

Bosworth, W., Off-axis volcanism in the Gregory rift, east Africa: Implications for models of continental rifting, $\mathrm{Ge}-$ ology, 15, 392-400, 1987.

Bosworth, W., J. Lambiase, and R. Keisler, A new look at Gregory's rift: The structural style of continental rifting, Eos, Trans. AGU, 67, 577, 1986.

Buck, W.R., Small-scale convection and the evolution of the lithosphere, Ph.D. thesis, 256 pp., Mass. Inst. Technol., Cambridge, 1984.

Buck W.R., Small scale convection induced by passive rifting: The cause for uplift of rift shoulders, Earth Planet. Sci. Lett., 77, 362-372, 1986.

Buck, W.R., F. Martinez, M.S. Steckler, and J.R. Cochran, Thermal consequences of lithospheric extension: Pure and simple, Tectonics, 7, 213-234, 1988.

Cochran, J.R., The Gulf of Aden: Structure and evolution of a young ocean basin and continental margin, J. Geophys. Res., 86, 263-288, 1981.

Cochran, J.R., A model for the development of the Red Sea, Am. Assoc. Pet. Geol. Bull., 67, 41-69, 1983a.

Cochran, J.R., Effects of finite rifting times on the development of sedimentary basins, Earth Planet. Sci. Lett., 66, 289-303, 1983b.

Cochran, J.R., and F. Martínez, Evidence from the northern Red Sea on the transition from continental rifting to seafloor spreading, Tectonophysics, 153, 25-53, 1988.

Cochran, J.R., F. Martínez, M.S. Steckler, and M.A. Hobart, Conrad Deep: A new northern Red Sea deep. Origin and implications for continental rifting, Earth Planet. Sci. Lett., 78, 18-32, 1986.

Coleman, R.G., The Red Sea: A small ocean basin formed by continental extension and seafloor spreading, in Origin and
History of Marginal and Inland Seas, Proc. 27th Int. Geol. Cong., vol. 23, 93-121, 1984.

Coleman, R.G., R.T. Gregory, and G.F. Brown, Cenozoic volcanic rocks of Saudi Arabia, U.S. Geol. Surv. Open File Rep., 83-788, 82 pp., 1983.

Colletta, B., P. Le Quellec, J. Letouzey, and I. Moretti, Longitudinal evolution of the Suez Rift structure [Egypt], Tectonophysics, 153, 221-233, 1988.

Courtillot, V., R. Armijo and P. Tapponnier, Kinematics of the Sinai triple junction and a two-phase model of ArabiaAfrica rifting, in Continental Extensional Tectonics, edited by M.P. Coward, J.F. Dewey, and P.L. Harcock, Geol. Soc. London. Spec. Publ. 28, 559-573,1987.

Coward, M.P., Heterogeneous stretching, simple shear and basin development, Earth Planet. Sci. Lett., 80, 325-336, 1986.

Crane, K., The spacing of rift axis highs: Dependence upon diapiric processes in the underlying asthenosphere?, Earth Planet. Sci. Lett., 72, 405-414, 1985.

Dunbar, J.A., and D.S. Sawyer, Continental rifting at pre-existing lithospheric weaknesses, Nature, $\underline{333}, 450-452$, 1988.

Ebinger, C.J., M.J. Crow, B.R. Rosendahl, D.A. Livingstone, and J. LeFournier, Structural Evolution of Lake Malawi, Africa, Nature, 308, 627-629, 1984.

Engel, A.E.J., T.H. Dixon, and R.J. Stern, Late Precambrian evolution of Afro-Arabian crust from ocean arc to craton, Geol. Soc. Am. Bull., 91, 699-706, 1980.

Erickson, A.J., R.P. von Herzen, J.G. Sclater, R.W. Girdler, B.V. Marshall, and R. Hyndman, Geothermal measurements in deep-sea drill holes, J. Geophys. Res., $\underline{80}, 2515$ 2528, 1975.

Freund, R., Z. Garfunkel, I. Zak, M. Goldberg, T. Weissbrod, and B. Derin, The shear along the Dead Sea Rift, Philos. Trans. R. Soc. London, Ser. A, 267, 107-130, 1970.

Furlong, K.P., and D.M. Fountain, Continental crustal underplating: Thermal considerations and seismic petrologic consequences, J. Geophys. Res., 91, 8285-8294, 1986.

Garfunkel, Z., Internal structure of the Dead Sea leaky transform [rift] in relation to plate kinematics, Tectonophysics, 80, 81-108, 1981.

Garfunkel, Z., and Y. Bartov, The tectonics of the Suez rift, Bull. Isr. Geol. Surv., 71, 44 p., 1977.

Garfunkel, Z., A. Ginzburg, and R.C. Searle, Fault pattern and mechanism of crustal spreading along the axis of the Red Sea from side scan [GLORIA] data, Ann. Geophys. 5B, 187-200, 1987.

Gaulier, J.M., X. LePichon, N. Lyberis, F. Avedik, L. Beli, I. Moretti, A. Deschamps, and S. Hafez, Seismic study of the crustal thickness, northern Red Sea and Gulf of Suez, Tectonophysics, $153,55-88,1988$.

Girdler, R.W., Problems conceming the evolution of oceanic lithosphere in the northern Red Sea, Tectonophysics, 116, 109-122, 1985.

Girdler, R.W., and T.R. Evans, Red Sea heat flow, Geophys. J. R. Astron. Soc., 51, 245-251, 1977.

Girdler, R.W., A.J. Erickson, and R. von Herzen, Downhole temperature and shipboard thermal conductivity measurements aboard D/V Glomar Challenger in the Red Sea, Initial Rep. Deep Sea Drill. Proj., 23, 879-886, 1974.

Goetze, C., and B. Evans, Stress and temperature in the bending lithosphere as constrained by experimental rock mechanics, Geophys. J. R. Astron. Soc., 59, 463-478, 1979.

Guennoc, P., G. Pautot, and A. Coutelle, Surficial structures of the northern Red Sea axial valley from $23^{\circ} \mathrm{N}$ to $28^{\circ} \mathrm{N}$ : Time and space evolution of neo-oceanic structures, Tectonophysics, 153 1-23, 1988.

Haenel, R., Heat flow measurements in the Red Sea and Gulf of Aden, Z. Geophys., 38, 1035-1047, 1972.

Herman, Y., and P.E. Rosenberg, Mineralogy and micropale- 
ontology of a goethite-bearing Red Sea core, in Hot Brines and Recent Heavy Metal Deposits in the Red Sea, edited by E.T. Degens, and D.A. Ross, pp. 448-459, Springer-Verlag, New York, 1969.

Heybroek, F., The Red Sea Miocene evaporite basin, in Salt Basins Around Africa, pp. 17-40, London Institute of Petroleum., 1965.

Hobart, M.A., J.R. Cochran, F. Martinez, and M.S. Steckler, The northern Red Sea, III. Heat flow and thermal state of the lithosphere, (abstract), Eos, Trans. AGU, 66, 365, 1985.

Izzeldin, A.Y., On the structure and evolution of the Red Sea, Ph.D. thesis, 163 pp., Univ. Louis Pasteur, Strasbourg, France, 1982.

Joffe, S., and Z. Garfunkel, Plate kinematics of the circum Red Sea - A re-evaluation, Tectonophysics, 141, 5-23, 1987.

Kirby, S.H., Rheology of the lithosphere, Rev. Geophys. 21, 1458-1487, 1983.

Knott, S.T., E.T. Bunce, and R.L. Chase, Red Sea seismic reflection studies, in The World Rift System, Geol. Surv. Can. Spec. Pap., 66, 33-61, 1966.

Kusznir, N.J., and R.G. Park, The extensional strength of the continental lithosphere: Its dependence on geothermal gradient and crustal composition and thickness, in Continental Extensional Tectonics, Spec. Publ. 28, Geol. Soc. London, edited by M.P. Coward, J. F. Dewey, and P.L. Harcock, 35-52, 1987.

Langseth, M.G., M.A. Hobart, and K. Horai, Heat flow in the Bering Sea, J. Geophys. Res., 85, 3740-3750, 1980.

LASE, Deep structure of the U.S. East Coast passive margin from large aperture seismic experiments [LASE], Mar. Pet. Geol., 3, 234-244, 1987.

Laughton, A.S., R.B. Whitmarsh, and M.T. Jones, The evolution of the Gulf of Aden, Philos. Trans, R. Soc. London., Ser. A, 267, 227-266, 1970.

LePichon, X., and J. Francheteau, A plate tectonic analysis of the Red Sea-Gulf of Aden area, Tectonophysics, 46, 369406, 1978.

LePichon, X., and J.M. Gaulier, The rotation of Arabia and the Levant fault system, Tectonophysics, 153, 271-294, 1988.

Levy, S., and R. Riddihough, Why are marine magnetic anomalies surpressed over sedimented spreading centers?, Geology, 14, 651-654, 1986.

Lowell, J.D., and G.J. Genik, Seafloor spreading and structural evolution of the southern Red Sea, Am. Assoc. Pet. Geol. Bull., 56, 247-259, 1972.

Makris, J., A. Allam, and L. Möller, Deep seismic studies in Egypt and their interpretation, (abstract), Eos, Trans. AGU, $62,230,1981$.

Martínez, F., and J.R. Cochran, Structure and tectonics of the northern Red Sea: Catching a continental margin between rifting and drifting, Tectonophysics, 150, 1-32, 1988.

Miller, P.M., and H. Barakat, Geology of the Safaga Concession, northern Red Sea, Egypt, Tectonophysics, 153, 123136, 1988.

Miller, S.P., K.C. Macdonald, and P. Lonsdale, Near bottom magnetic profile across the Red Sea, Mar. Geophys. Res., $1,401-418,1985$.

Montadert, L., O. de Charpel, D.G. Roberts, P. Guennoc, and J. Sibuet, Northeast Atlantic passive continental margins: Rifting and subsidence processes, in Deep Drilling Results in the Atlantic Ocean: Continental Margins and Paleoenvironment, Maurice Ewing Ser., vol. 3, edited by M. Talwani, W. Hey, and W.B.F. Ryan, pp. 154-186 AGU, Washington, D.C., 1979.

Morgan, P., F.K. Boulos, S.F. Hennin, A.A. El-Sheriff, A.A. El-Sayed, N.Z. Basta, and Y.S. Melek, Heat flow in eastern Egypt: The thermal signature of a continental breakup, J. Geodyn. 4, 107-131, 1985.

Moustafa, A.M., Block faulting in the Gulf of Suez, Fifth Ex- ploration Seminar, Egypt. Gen. Pet. Corp. 36 pp., Cairo, 1976.

Murrell, S.A.F., Rheology of the lithosphere-Experimental indications, Tectonophysics, 36, 5-24, 1976.

Mutter, J.C., W.R. Buck, and C.M. Zehnder, Convective partial melting 1. A model for the formation of thick basalt sequences during the initiation of spreading, J. Geophys. Res., 93, 1031-1048, 1988.

Parsons, B., and J.G. Sclater, An analysis of ocean floor bathymetry and heat flow with age, J. Geophys. Res., $\underline{82}$, 803-827, 1977.

Pautot, G., Les fosses de la Mer Rouge: Approche géomorphologique d'un stade initial d'ouvertur océanique réalisée á l'aide du Seabeam, Oceanol. Acta, 6, 235-244, 1983.

Pautot, G., P. Guennoc, A. Coutelle, and N. Lyberis, Discovery of a large brine deep in the northern Red Sea, Nature, 310, 133-136, 1984.

Pautot, G., P. Guennoc, A. Coutelle, and N. Lyberis, La dépression axiale du segment nord Mer Rouge [de $25^{\circ} \mathrm{N}$ á $28^{\circ} \mathrm{N}$ ]: Nouvelles donnés géologiques et geophysiques obtenues au cours de la campagne Transmerou 83, Bull. Soc. Géol. Fr., 8, 381-399, 1986.

Phillips, J.D., and D.A. Ross, Continuous seismic reflection profiles in the Red Sea, Philos. Trans. R. Soc. London, Ser. A, 267, 143-152, 1970.

Ratcliffe, E.H., The thermal conductivities of ocean sediments, J. Geophys. Res., 65, 1535-1541, 1960.

Roeser, H.A., A detailed magnetic survey of the southern Red Sea, Geol. Jahrb., 13, 131-153, 1975.

Rowley, D.B., and D. Sahagian, Depth-dependent stretching: A different approach, Geology, 14, 32-35, 1986.

Royden, L., and C.E. Keen, Rifting processes and thermal evolution of the continental margin of eastern Canada determined from subsidence curves, Earth Planet. Sci. Lett., 51, 343-361, 1980.

Searle, R.C., and D.A. Ross, A geophysical study of the Red Sea axial trough between $20.5^{\circ}$ and $22^{\circ} \mathrm{N}$, Geophys. J. R. Astron. Soc., 43, 555-572, 1975.

Sleep, N.H., Thermal effects of formation of Atlantic continental margins by continental breakup, Geophys. J. R. Astron. Soc., 24, 325-350, 1971.

Steckler, M.S., Uplift and extension in the Gulf of Suez, indications of induced mantle convection, Nature, $\underline{317}$, 135-139, 1985.

Steckler, M.S., F. Berthelot, N. Lyberis and X. LePichon, Subsidence in the Gulf of Suez: Implications for rifting and plate kinematics, Tectonophysics, $153,249-270,1988$.

Stern, R.J., Petrogenesis and tectonic setting of late Precambrian ensimatic volcanic rocks, Eastern Desert of Egypt, Precambrian Res., 16, 195-230, 1981.

Stern, R.J., D. Gottfried, and C.E. Hedge, Late Precambrian rifting and crustal evolution in the northeastern desert of Egypt, Geology, 12, 168-172, 1984.

Stoeser, D.B., and V.E. Camp, Pan-African microplate accretion of the Arabian Shield, Geol. Soc. Am. Bull., 96, 817$826,1985$.

Stoffers, P., and D.A. Ross, Sedimentary history of the Red Sea, Initial Rep. Deep Sea Drill. Proj., 23, 849-866, 1974.

Tewfik, N., and M. Ayyad, Petroleum exploration in the Red Sea shelf of Egypt, Sixth Exploration Seminar, Egypt. Gen. Pet. Corp., Cairo, 30 pp., 1982.

Touloukian, Y.S., and C.Y. Ho. (Eds.), Physical Properties of Rocks and Minerals, McGraw-Hill/Cindas Data Series on Material Properties, New York, 1981.

Tramontini, C., and D. Davies, A seismic refraction survey in the Red Sea, Geophys. J. R. Astron. Soc., 17, 225-241, 1969.

von Herzen, R., and A.E. Maxwell, The measurement of thermal conductivity of deep-sea sediments by a needleprobe method, J. Geophys. Res., 64, 1557-1563, 1959.

Wall, D., and J.S. Warren, Dinoflagellates in Red Sea piston cores, in Hot Brines and Recent Heavy Metal Deposits in 
the Red Sea, edited by E.T. Degens, and D.A. Ross, pp. 317-328, Springer-Verlag, New York, 1969.

Watts, A.B., and M.S. Steckler, Subsidence and tectonics of Atlantic-type continental margins, Oceanol. Acta, 4, 143$153,1981$.

Weissel, J.K., and G. Karner, Flexural uplift of rift flanks due to tectonic denudation of the lithosphere during extension, J. Geophys. Res., in press, 1989.

Wernicke, B., Uniform-sense normal simple shear of the continental lithosphere, Can. J. Earth Sci., 22, 108-125, 1985.

Whieldon, J., T.R. Evans, and R.W. Girdler, Thermal conductivity, density and sonic velocity measurements of samples of anhydrite and halite from sites 225 and 227, Initial Rep. Deep Sea Drill. Proj., 23, 909-912, 1974.

White, N., and D. McKenzie, Formation of steer's head ge- ometry of sedimentary basins by differential stretching of the crust and mantle, Geology, 16, 250-253, 1988.

Wyrtki, K., Oceanographic Atlas of the International Indian Ocean Expedition, U.S. Goverment Printing Office, Washington, D.C., 1971.

J.R. Cochran, Lamont-Doherty Geological Observatory, Palisades, NY 10964

F. Martinez, Hawaii Institute of Geophysics, 2525 Correa Road, Honolulu, HI 96822

(Received September 13, 1988;

revised March 28, 1989;

accepted December 28, 1988.) 